\title{
All Killing superalgebras for warped AdS backgrounds
}

\author{
S. Beck, ${ }^{a}$ U. Gran, ${ }^{b}$ J. Gutowski ${ }^{c}$ and G. Papadopoulos ${ }^{a}$ \\ ${ }^{a}$ Department of Mathematics, King's College London, \\ Strand, London WC2R 2LS, U.K. \\ ${ }^{b}$ Department of Physics, Division for Theoretical Physics, Chalmers University of Technology, \\ SE-412 96 Göteborg, Sweden \\ ${ }^{c}$ Department of Mathematics, University of Surrey, \\ Guildford, GU2 7XH, U.K. \\ E-mail: samuel.beck@kcl.ac.uk, ulf.gran@chalmers.se, \\ j.gutowski@surrey.ac.uk, george.papadopoulos@kcl.ac.uk
}

ABStRACT: We present all the symmetry superalgebras $\mathfrak{g}$ of all warped $\operatorname{AdS}_{k} \times_{w} M^{d-k}$, $k>2$, flux backgrounds in $d=10,11$ dimensions preserving any number of supersymmetries. First we give the conditions for $\mathfrak{g}$ to decompose into a direct sum of the isometry algebra of $\mathrm{AdS}_{k}$ and that of the internal space $M^{d-k}$. Assuming this decomposition, we identify all symmetry superalgebras of $\mathrm{AdS}_{3}$ backgrounds by showing that the isometry groups of internal spaces act transitively on spheres. We demonstrate that in type II and $d=11$ theories the $\mathrm{AdS}_{3}$ symmetry superalgebras may not be simple and also present all symmetry superalgebras of heterotic $\mathrm{AdS}_{3}$ backgrounds. Furthermore, we explicitly give the symmetry superalgebras of $\mathrm{AdS}_{k}, k>3$, backgrounds and prove that they are all classical.

Keywords: Flux compactifications, Space-Time Symmetries, Supergravity Models, Superstring Vacua

ArXiv ePrint: 1710.03713 


\section{Contents}

1 Introduction 1

2 Killing superalgebras $\quad 4$

2.1 Definition of KSAs 4

2.2 AdS Killing spinors 4

2.3 1-form bilinears and decomposition of $\mathfrak{g}_{0} \quad 6$

$\begin{array}{lll}2.4 & \text { Global conditions for the decomposition of } \mathfrak{g}_{0} & 7\end{array}$

$3 \mathrm{AdS}_{3}$ in $\mathrm{D}=11$ and type II theories $\quad 7$

$\begin{array}{lll}3.1 \quad N=2 & 7\end{array}$

$3.2 \quad N=4 \quad 8$

$\begin{array}{lll}3.2 .1 & \text { Left and right superalgebra } & 8\end{array}$

$\begin{array}{ll}3.2 .2 & \text { Left or right superalgebra }\end{array}$

$\begin{array}{lll}3.3 & \text { Some structure theory } & 10\end{array}$

3.3.1 Structure constants of KSAs 10

3.3.2 The $\mathrm{AdS}_{3}$ KSAs are direct sums of Left and Right KSAs 11

$\begin{array}{lll}3.3 .3 & \text { Structure theorems } & 12\end{array}$

3.4 KSAs from $\mathfrak{s o}(n)$ acting transitively on $S^{n-1} \quad 14$

3.4.1 Generic case $N=N_{\sigma}=2 n \quad 14$

3.4.2 $N=N_{\sigma}=8 \quad 14$

$\begin{array}{lll}\text { 3.5 KSAs from } \mathfrak{u}(n) \text { acting transitively on } S^{2 n-1} & 15\end{array}$

$\begin{array}{lll}3.6 & \text { KSAs from } \mathfrak{g}_{2} \text { and } \mathfrak{s p i n}(7) & 16\end{array}$

3.7 KSAs from $\mathfrak{s p}^{*}(n) \oplus \mathfrak{s p}^{*}(1)$ and the remaining cases 17

$\begin{array}{lll}3.7 .1 & \mathfrak{s p i n}(9), \mathfrak{s p}^{*}(n) \text { and } \mathfrak{s p}^{*}(n) \oplus \mathfrak{u}(1) & 17\end{array}$

$\begin{array}{lll}3.7 .2 & \mathfrak{s p}^{*}(n) \oplus \mathfrak{s p}^{*}(1) & 18\end{array}$

4 Heterotic backgrounds $\quad 19$

$\begin{array}{lll}4.1 & \text { Killing spinors } & 19\end{array}$

$\begin{array}{lll}4.2 & \text { KSAs for heterotic backgrounds } & 20\end{array}$

$5 \mathrm{AdS}_{4}$ in $\mathrm{D}=11$ and type II theories $\quad 21$

$\begin{array}{lll}5.1 N=4 & 21\end{array}$

$\begin{array}{lll}5.2 N=8 & 22\end{array}$

$\begin{array}{lll}5.3 \quad N=12 & 22\end{array}$

$\begin{array}{lll}5.4 \quad N=16 & 23\end{array}$

$\begin{array}{lll}5.5 & N>16 & 23\end{array}$

$6 \quad \mathrm{AdS}_{5}$ in $\mathrm{D}=11$ and type II theories $\quad 24$

$\begin{array}{lll}6.1 & N=8 & 24\end{array}$

$6.2 \quad N=16 \quad 25$

$\begin{array}{lll}6.3 & \text { Extended supersymmetry } & 26\end{array}$ 
$8 \mathrm{AdS}_{7}$ in $\mathrm{D}=11$ and type II theories 28

9 Conclusions $\quad 30$

A Invariance of (massive) IIA fluxes $\quad 32$

$\begin{array}{lll}\text { B AdS superalgebra } & 34\end{array}$

B.1 Isometries of AdS 34

B.2 Spinorial Lie derivatives along AdS 35

C Commutators $\quad 36$

$\begin{array}{lll}\text { C.1 } & \text { 1-form bilinears } & 36\end{array}$

$\begin{array}{lll}\text { C.2 Spinorial Lie derivative } & 38\end{array}$

$\begin{array}{lll}\mathrm{D}^{\mathrm{AdS}_{3}} \mathrm{KSAs} \text { for } N<14 & 39\end{array}$

D.1 $N=N_{\sigma}=10 \quad 39$

D.2 $N=N_{\sigma}=12$

\section{Introduction}

One way to find the symmetry superalgebra, $\mathfrak{g}=\mathfrak{g}_{0} \oplus \mathfrak{g}_{1}$, of a product $A d S_{k} \times M^{d-k}$, $k>2$, background in a supergravity theory is to assume that it is a classical superalgebra ${ }^{1}$ whose even subalgebra decomposes as $\mathfrak{g}_{0}=\mathfrak{s o}(k-1,2) \oplus \mathfrak{t}_{0}$ and the dimension of the odd subspace $\mathfrak{g}_{1}$ is the number of Killing spinors $N$, where $\mathfrak{s o}(k-1,2)$ is the Lie algebra of isometries of $\mathrm{AdS}_{k}$ subspace. Then $\mathfrak{t}_{0}$ is identified with the Lie algebra of isometries of the internal space $M^{d-k}$. For $k>3$, these data together with the classification of classical superalgebras in $[1,2]$ are sufficient to find all such symmetry superalgebras.

This method based on the splitting and classification of classical superalgebras may be sufficient for backgrounds of the type $A d S_{k} \times M^{d-k}$ but that is not the case for generic warped $A d S_{k} \times{ }_{w} M^{d-k}$ solutions. This is because $A d S_{k}$ can be written as a warped product of $A d S_{m}$ for any $m<k$ and so all $A d S_{k} \times{ }_{w} M^{d-k}$ backgrounds can be reinterpreted as $A d S_{m} \times{ }_{w} M^{d-m}$ backgrounds [3, 4]. Now if all the symmetry supergralgebras of AdS backgrounds could be identified as described above, it would have been possible to decompose the even subalgebra $\mathfrak{g}_{0}$ of the symmetry superalgebra of $A d S_{k} \times{ }_{w} M^{d-k}$ as $\mathfrak{g}_{0}=\mathfrak{s o}(m-1,2) \oplus \mathfrak{t}_{0}^{\prime}$. However in all known examples this is not the case. Therefore, there must be some conditions on the spacetime geometry required for $\mathfrak{g}_{0}$ to decompose as $\mathfrak{s} o(k-1,2) \oplus \mathfrak{t}_{0}$. Furthermore it is not a priori obvious why one should restrict the symmetry superalgebras of AdS backgrounds to be classical.

\footnotetext{
${ }^{1}$ The classical superalgebras are those which are simple and where the representation of $\mathfrak{g}_{0}$ on $\mathfrak{g}_{1}$ is completely reducible [1].
} 
First principle computations of $\mathfrak{g}$ have also been made in the literature for many known supersymmetric solutions, see e.g. [5] and [6-8]. Most of these are based on the Killing superalgebra (KSA) approach [7,9] which utilizes the geometric data of the spacetime like the Killing spinor 1-form bilinears and the spinorial Lie derivative to define the (anti-) commutators of $\mathfrak{g}$; the method is reviewed in section 2 . In these computations, the geometry of the internal space $M^{d-k}$ is used in an essential way to determine all (anti-) commutators. Because of this, it is not apparent how to extend to general warped $A d S_{k} \times{ }_{w} M^{d-k}$ flux backgrounds where the geometry of the internal space $M^{d-k}$ may not be sufficiently known to find the (anti-)commutators of $\mathfrak{g}$.

In this paper we shall apply a modification of the KSA approach to identify all the symmetry superalgebras of warped AdS backgrounds $A d S_{k} \times{ }_{w} M^{d-k}$ with the most general allowed fluxes in 10- and 11-dimensional supergravity theories. First, we find the conditions on the geometry of $A d S_{k} \times_{w} M^{d-k}$ such that $\mathfrak{g}_{0}$ can admit a decomposition ${ }^{2}$ as $\mathfrak{g}_{0}=$ $\mathfrak{s} o(k-1,2) \oplus \mathfrak{t}_{0}$. These conditions are expressed as vanishing conditions for certain Killing spinor bilinears or their derivatives and are stated in eqs. (2.9), (2.10) and (2.12). We also demonstrate that the same conditions can be derived if one assumes that the internal space is compact without boundary and the solutions are smooth.

Next we find that for $\mathrm{AdS}_{3}$ backgrounds the KSA decomposes as $\mathfrak{g}=\mathfrak{g}_{L} \oplus \mathfrak{g}_{R}$, where $\mathfrak{g}_{L}$ is associated to the left action on $\mathrm{AdS}_{3}$ and $\mathfrak{g}_{R}$ is associated with the right-action on $\mathrm{AdS}_{3}$ viewed locally as a group manifold. For $N<8$ superymmetries in either the left or the right sector, the KSAs can be computed from first principles. The method we use will be explained later. For $N \geq 8$ in either the left or the right sector, we show that the isometry algebra of the internal space acts transitively on a sphere in $\mathfrak{g}_{1}$ and leaves a 4 -form invariant. Moreover we show that all the structure constants of the KSA can be determined as soon as this 4 -form is specified. The classification of groups acting transitively and effectively on spheres has been solved some time ago in [10] and has been used [11] in the context of the Berger classification of irreducible simply connected Riemannian manifolds. Using this, all KSAs of $A d S_{3}$ backgrounds are found and the results are tabulated in table 2. The table includes three series $\mathfrak{o s p}(N / 2 \mid 2), \mathfrak{s l}(N / 2 \mid 2)$ and $\mathfrak{o s p} *(N / 4 \mid 4)(N=16,24),{ }^{3}$ as well as several exceptional cases like $\mathfrak{D}(2,1, \alpha)(N=8), \mathfrak{g}(3)(N=14)$ and $\mathfrak{f}(4)(N=16)$, where $N=\operatorname{dim} \mathfrak{g}_{1}$ is the number of supersymmetries. It is also shown that the KSAs of $\mathrm{AdS}_{3}$ backgrounds are not necessarily simple as they can exhibit central terms. Though to our knowledge there are no solutions in the literature for which a central term gives rise to an effective action on the internal space.

We also identify the KSAs of heterotic $\mathrm{AdS}_{3}$ backgrounds. The results are presented in table 3 . The KSAs of heterotic $\mathrm{AdS}_{3}$ backgrounds are of classical type and thus they do not exhibit central generators.

\footnotetext{
${ }^{2}$ One justification for this decomposition is AdS/CFT. The isometry group of the AdS and internal spaces are identified with the conformal and R-symmetry groups of the dual theory, respectively. As the conformal and R-symmetry groups of a field theory commute, $\mathfrak{g}_{0}$ must be a direct sum. This is the only assumption we make.

${ }^{3} \mathfrak{g}^{*}$ denotes another real form of the real superalgebra $\mathfrak{g}$.
} 
Applying the same methods to the rest of $A d S_{k} \times{ }_{w} M^{d-k}, k>3$ backgrounds, we demonstrate that the KSAs are of classical type. There is only one exception to this which is the KSA of maximally supersymmetric $\mathrm{AdS}_{5}$ backgrounds which allows the presence of a central term. However, as we know that the only maximally supersymmetric $\mathrm{AdS}_{5}$ solution is locally isometric to the $A d S_{5} \times S^{5}$ solution in IIB, one can show that the central term does not act effectively on $S^{5}$ and so it can be set to zero. The KSAs for all $A d S_{k} \times{ }_{w} M^{d-k}$ $k>3$ backgrounds are explicitly constructed and are related to the classification of the classical superalgebras in [1,2]. The list of the KSAs of $A d S_{k} \times{ }_{w} M^{d-k}, k>3$ can be found in table 4 and the associated isometry algebras of the internal spaces in table 5.

There are two key developments that have allowed us to prove these results without specifying the geometry of the internal spaces. The first is the explicit expression of the Killing spinors of $A d S_{k} \times{ }_{w} M^{d-k}$ backgrounds given in [12-14] for which the dependence on the AdS coordinates is manifest. ${ }^{4}$ As a result, one can determine the dependence of all 1-form Killing spinor bilinears on the AdS coordinates and to also compute explicitly all the spinorial Lie derivatives of the Killing spinors along the isometries of AdS. As a consequence, one can determine the anti-commutator $\left\{\mathfrak{g}_{1}, \mathfrak{g}_{1}\right\}$ as well as all the commutators, $\left[\mathfrak{s o}(k-1,2), \mathfrak{g}_{1}\right]$, of the odd generators of the KSA with the even generators associated with isometries of $\operatorname{AdS}_{k}, \mathfrak{s o}(k-1,2) \subseteq \mathfrak{g}_{0}$. Furthermore, it is straightforward to find the conditions (2.9), (2.10) and (2.12) for the even part of the superalgebra to decompose as a direct sum of the the isometry algebra of AdS space and that of the internal space, $\mathfrak{g}_{0}=\mathfrak{s o}(k-1,2) \oplus \mathfrak{t}_{0}$. These conditions put several restrictions on the geometry of $A d S_{k} \times{ }_{w} M^{d-k}$. In particular for $k>3$, they can be used to find the linearly independent Killing vectors of the internal space and in this way determine the dimension of $\mathfrak{t}_{0}$. For $k=3$, these are sufficient to determine the maximal dimension of $\mathfrak{t}_{0}$.

The second ingredient in our proof is the closure of KSAs for superymmetric $d=11$ and IIB backgrounds shown in $[15,16]$. We use this to demonstrate that in all cases the superJacobi identities and the explicit dependence of the Killing spinors on the AdS coordinates are sufficient to determine the commutator $\left[\mathfrak{t}_{0}, \mathfrak{g}_{1}\right]$ from those of $\left\{\mathfrak{g}_{1}, \mathfrak{g}_{1}\right\}$ and $\left[\mathfrak{s o}(k-1,2), \mathfrak{g}_{1}\right]$. This circumvents the need to know details of the geometry of the internal spaces in order to find the KSAs. The remaining commutators which are those of the isometries of the internal space can also be found after applying the super-Jacobi identities.

This paper is organized as follows. In section 2, we summarize the results of [12-14] and use them to find the conditions on the geometry of $A d S_{k} \times_{w} M^{d-k}$ such that $\mathfrak{g}_{0}=$ $\mathfrak{s o}(k-1,2) \oplus \mathfrak{t}_{0}$. In section 3, we classify all KSAs for $\mathrm{AdS}_{3}$ backgrounds. In section 4, we determine all KSAs for heterotic $\mathrm{AdS}_{3}$ backgrounds. In sections 5, 6, 7 and 8, we show that the KSAs of $\operatorname{AdS}_{k}, k=4,5,6$ and 7 are classical and give explicitly all their (anti-)commutators, respectively. In section 9, we give our conclusions. In appendix A, we demonstrate that the 1-form Killing spinor bilinears of massive IIA supergravity leave invariant all fields of the theory. In appendix B, we give the isometries of $\mathrm{AdS}_{k}$ as well as the spinorial Lie derivatives of spinors along the AdS Killing vectors. In appendix C, we

\footnotetext{
${ }^{4}$ It has also removed all the assumptions that are usually made on the form of the Killing spinors for $A d S_{k} \times{ }_{w} M^{d-k}$ backgrounds which have been proven to be restrictive in [4].
} 
give all the 1-form bilinears as well as the spinorial Lie derivatives of the Killing vectors of $A d S_{k} \times{ }_{w} M^{d-k}$ backgrounds. In appendix D, we present the construction of KSAs for $\mathrm{AdS}_{3}$ backgrounds with a low number of supersymmetries without the use of the results of [10].

\section{Killing superalgebras}

\subsection{Definition of KSAs}

Decomposing the KSAs of supersymmetric backgrounds $\mathfrak{g}$ into the even $\mathfrak{g}_{0}$ and odd subspaces $\mathfrak{g}_{1}, \mathfrak{g}=\mathfrak{g}_{0} \oplus \mathfrak{g}_{1}$, the construction proceeds as follows [7,9]. $\mathfrak{g}_{1}$ is spanned by the odd generators $Q_{\epsilon_{\mathbf{m}}}$ each associated to a Killing spinor $\epsilon_{\mathbf{m}}$ of the background, where $\mathbf{m}=1, \ldots N . \mathfrak{g}_{0}$ is spanned by the even generators $V_{K_{\mathbf{m n}}}$ each associated to a 1 -form bilinear $K_{\mathbf{m n}}$ constructed from the Killing spinors $\epsilon_{\mathbf{m}}$ and $\epsilon_{\mathbf{n}}$ as $K_{\mathbf{m n}}=\left(\epsilon_{\mathbf{m}}, \Gamma_{M} \epsilon_{\mathbf{n}}\right) d x^{M}$, where (, ) is a suitable $\operatorname{Spin}(d-1,1)$-invariant inner product such that $K_{\mathbf{m n}}=K_{\mathbf{n m}} \cdot K_{\mathbf{m n}}$ give rise to Killing vector fields which leave all fields invariant. The (anti)commutators of KSAs are computed geometrically. In particular

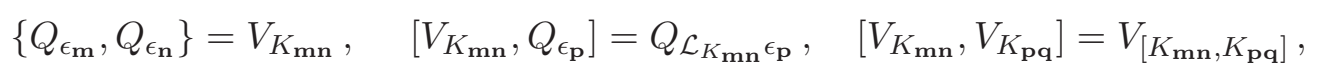

where $\left[K_{\mathbf{m n}}, K_{\mathbf{p q}}\right]$ is the Lie commutator of two vector fields and

$$
\mathcal{L}_{X} \epsilon=\nabla_{X} \epsilon+\frac{1}{8} d X_{M N} \Gamma^{M N} \epsilon
$$

is the spinorial Lie derivative of $\epsilon$ with respect to the vector field $X$. It has been shown in $[15,16]$ that closure of the KSAs holds for all $d=11$ and IIB supersymmetric backgrounds ${ }^{5}$ under these operations as the super-Jacobi identities are satisfied. There are several simplifications in the construction of the KSAs for AdS backgrounds which we shall explain below. In what follows, we shall set for simplicity $Q_{\epsilon_{\mathbf{m}}}=Q_{\mathbf{m}}$ and $V_{K_{\mathbf{m n}}}=V_{\mathbf{m n}}$.

\subsection{AdS Killing spinors}

The KSEs of all warped backgrounds $\operatorname{AdS}_{k} \times{ }_{w} M^{d-k}, k \geq 3$, with the most general allowed fluxes can be integrated over the AdS subspace in all 10- and 11-dimensional supergravity theories [12-14]. The expression for the fluxes depends on the theory as well as the particular $\mathrm{AdS}_{k}$ background under consideration. However, the properties that will be described apply to all cases. Because of this in what follows we shall focus on the metric which is universal in all theories and for the rest one should consult the references above. In the coordinates that the spacetime metric can be written as

$$
d s^{2}=2 d u\left(d r-2 \ell^{-1} r d z-2 r d \ln A\right)+A^{2} d z^{2}+A^{2} e^{2 z / \ell} \delta_{a b} d x^{a} d x^{b}+g_{I J} d y^{I} d y^{J},
$$

where $\left(u, r, z, x^{a}\right)$ are coordinates of the AdS subspace, $\ell$ is the radius of AdS, $y$ are coordinates of $M$ and $A$ is the warp factor, the expression for Killing spinors reads

$$
\epsilon=\epsilon_{1}+\epsilon_{2}+\epsilon_{3}+\epsilon_{4},
$$

\footnotetext{
${ }^{5}$ Closure has not been shown for the KSAs of massive IIA backgrounds but it is expected to hold for those as well. In appendix A, we demonstrate that the vector Killing spinor bilinears of massive IIA theory leave all fields of the theory invariant.
} 
where

$$
\begin{aligned}
& \epsilon_{1}=\sigma_{+}, \quad \epsilon_{2}=\sigma_{-}-\ell^{-1} e^{\frac{z}{\ell}} x^{a} \Gamma_{a z} \sigma_{-}-\ell^{-1} A^{-1} u \Gamma_{+z} \sigma_{-}, \\
& \epsilon_{3}=e^{-\frac{z}{\ell}} \tau_{+}-\ell^{-1} A^{-1} r e^{-\frac{z}{\ell}} \Gamma_{-z} \tau_{+}-\ell^{-1} x^{a} \Gamma_{a z} \tau_{+}, \quad \epsilon_{4}=e^{\frac{z}{\ell}} \tau_{-},
\end{aligned}
$$

where the $\sigma_{ \pm}$and $\tau_{ \pm}$spinors satisfy the lightcone projections $\Gamma_{ \pm} \sigma_{ \pm}=\Gamma_{ \pm} \tau_{ \pm}=0$, and depend only on the coordinates of $M^{k}$ The gamma matrices have been chosen with respect to the frame

$$
\begin{array}{rlrl}
\mathbf{e}^{+} & =d u, & \mathbf{e}^{-} & =d r-2 \ell^{-1} r d z-2 r d \ln A, \\
\mathbf{e}^{z} & =A d z, & \mathbf{e}^{a}=A e^{\frac{z}{\ell}} d x^{a}, \quad \mathbf{e}^{i}=e_{I}^{i} d y^{I},
\end{array}
$$

and $d s^{2}=2 \mathbf{e}^{+} \mathbf{e}^{-}+\left(\mathbf{e}^{z}\right)^{2}+\delta_{a b} \mathbf{e}^{a} \mathbf{e}^{b}+\delta_{i j} \mathbf{e}^{i} \mathbf{e}^{j}$. The spinors $\sigma_{ \pm}$and $\tau_{ \pm}$satisfy some KSEs along the internal space $\mathrm{M}^{d-k}$ which are

$$
D_{i}^{( \pm)} \sigma_{ \pm}=D_{i}^{( \pm)} \tau_{ \pm}=0, \quad \mathcal{A}^{( \pm)} \sigma_{ \pm}=\mathcal{A}^{( \pm)} \tau_{ \pm}=0,
$$

and can be thought as the restriction of the gravitino and dilatino KSEs of the associated supergravity theory on $M^{d-k}$, respectively, as well as an additional algebraic KSE

$$
\Xi^{( \pm)} \sigma_{ \pm}=0, \quad \Xi^{( \pm)} \tau_{ \pm}=\mp \frac{1}{\ell} \tau_{ \pm}
$$

which arises from the integration of KSEs on AdS. The explicit form of (2.7) and (2.8) depends on the supergravity considered as well as the specific AdS background under investigation, as the operators $D_{i}^{( \pm)}, \mathcal{A}^{( \pm)}$and $\Xi^{( \pm)}$depend on the fields. However in all cases they take the above general form.

The $\sigma_{+}$and $\tau_{+}$Killing spinors lie in a complementary subspace from that of $\sigma_{-}$and $\tau_{-}$as they satisfy different lightcone projections. Moreover, it is straightforward to deduce from the algebraic KSE (2.8) that the $\sigma_{+}$Killing spinors are linearly independent from the $\tau_{+}$Killing spinors. From this analysis, one concludes that Killing spinors $\epsilon_{1}, \epsilon_{2}, \epsilon_{3}$ and $\epsilon_{4}$ are all linearly independent. Later, we shall strengthen these properties using global conditions.

For $\operatorname{AdS}_{k} k \geq 3$, there are elementary Clifford algebra operations that relate the $\sigma$ and $\tau$ spinors. In particular if $\sigma_{+}$and $\tau_{+}$are Killing spinors, then $\sigma_{-}=A \Gamma_{-z} \sigma_{+}$and $\tau_{-}=A \Gamma_{-z} \tau_{+}$are Killing spinors. In addition for $k>3$, if $\sigma_{+}$is a Killing spinor, then $\Gamma_{z a} \sigma_{+}$are $\tau_{+}$type Killing spinors for every $a$. Moreover for $k>4$ if $\sigma_{+}$is a Killing spinor, then $\Gamma_{a b} \sigma_{+}$are $\sigma_{+}$type Killing spinors for every $a<b$. These relations between the Killing spinors can be used to count the Killing spinors of all AdS backgrounds [12-14]. For $k=3$, it can be arranged so that for each $\epsilon$ only the $\sigma_{ \pm}$or the $\tau_{ \pm}$spinors are non-vanishing and also the terms proportional to $x^{a}$ in the expression for $\epsilon$ do not occur as the coordinates of $\mathrm{AdS}_{3}$ are $(u, r, z)$.

It is clear from the above that the Killing spinors of AdS backgrounds can be described in terms of multiplets. Each multiplet is determined from the choice of $\sigma_{+}$. Then the rest of the components of the multiplet can be constructed from $\sigma_{+}$using the elementary Clifford algebra operations described above and after selecting the linearly independent spinors 
that arise from such a procedure. AdS backgrounds that preserve a minimal amount of supersymmetry admit one such multiplet of Killing spinors while those that preserve extended supersymmetry admit two or more such multiplets. We shall investigate both the properties of the Killing spinors that lie in the same multiplet as well as those of different multiplets. It suffices to focus on the properties of $\sigma_{+}$and $\tau_{+}$Killing spinors as those of $\sigma_{-}$and $\tau_{-}$spinors follow in a straightforward manner.

\subsection{1-form bilinears and decomposition of $\mathfrak{g}_{0}$}

To investigate the conditions on the geometry of $\mathrm{AdS}_{k} \times{ }_{w} \mathrm{M}^{d-k}$ backgrounds for $\mathfrak{g}_{0}$ to decompose as $\mathfrak{g}_{0}=\mathfrak{s o}(k-1,2) \oplus \mathfrak{t}_{0}$, we use the 1-form bilinears of the Killing spinors (2.5) presented in appendix C. These have components along the AdS subspace and components along the internal space $\mathrm{M}^{d-k}$. The components along the AdS subspace span the 1forms associated with the isometries of the AdS subspace in (2.3). There are two kinds of components along the internal space $\mathrm{M}^{d-k}$, those that depend on and those that are independent from the coordinates of AdS. The former indicate that the backgrounds have isometries along the internal space which do not commute with the isometries of AdS. Such behavior is expected from $\mathrm{AdS}_{k}$ solutions that arise as foliations of $\mathrm{AdS}_{m}$ backgrounds with $k<m$. To exclude such backgrounds, it is required that all such components should vanish. In turn, it is straightforward to observe that this is satisfied provided that

$$
\left\langle\sigma_{+}, \Gamma_{i} \Gamma_{a} \sigma_{+}^{\prime}\right\rangle=0,\left\langle\tau_{+}, \Gamma_{i} \Gamma_{z} \sigma_{+}\right\rangle=0,
$$

where $\sigma_{+}^{\prime}, \tau_{+}$and $\sigma_{+}$may or may not belong to the same Killing spinor multiplet, and we have used the relation between $\tau_{-}, \sigma_{-}$and $\tau_{+}, \sigma_{+}$spinors. The inner product $\langle\cdot, \cdot\rangle$ is the real part of the standard hermitian inner product for which all spacelike gamma matrices are hermitian. For $k>3$, the above two relations are equivalent as there is a relation between the $\sigma_{+}$and $\tau_{+}$spinors explained in the previous section. The remaining 1-form bilinears along the internal space are always proportional to $\left\langle\sigma_{+}, \Gamma_{i} \Gamma_{z} \sigma_{+}^{\prime}\right\rangle \mathbf{e}^{i}$, where again $\sigma_{+}^{\prime}$ and $\sigma_{+}$may or may not belong to the same Killing spinor multiplet. These are not expected to vanish and give rise to isometries of the internal space.

One consequence of (2.9) is the orthogonality of $\sigma_{+}$and $\tau_{+}$spinors

$$
\left\langle\sigma_{+}, \tau_{+}\right\rangle=0
$$

This follows from (2.8) after imposing (2.9).

After imposing (2.9) and (2.10), the Killing spinor bilinears can be written as $K=$ $K_{\mu} \mathbf{e}^{\mu}+K_{i} \mathbf{e}^{i}$, where $K^{\mathrm{AdS}}=K_{\mu} \mathbf{e}^{\mu}$ are along the AdS directions and $\tilde{K}=K_{i} \mathbf{e}^{i}$ are along the transverse directions. $K^{\text {AdS }}$ can be written as a linear combination of forms associated with Killing vector fields of the AdS space with components that may depend on the coordinates of of the internal space. Requiring that $\mathfrak{g}_{0}=\mathfrak{s o}(k-1,2) \oplus \mathfrak{t}_{0}$ which implies that independently $K^{\mathrm{AdS}}$ and $\tilde{K}$ are Killing and that

$$
\left[K^{\mathrm{AdS}}, \tilde{K}\right]=0
$$


one finds that

$$
\left\|\sigma_{+}\right\|=\text {const }
$$

and

$$
K^{i} \partial_{i} A=0, \quad \tilde{\nabla}_{(i} K_{j)}=0,
$$

ie the length of the Killing spinors is constant, the warp factor is invariant and $\tilde{K}$ is Killing on the internal space $M^{d-k}$, where $\tilde{\nabla}$ is the Levi-Civita connection of the internal space $\mathrm{M}^{d-k}$. To summarize, the conditions for $\mathfrak{g}_{0}$ to decompose as $\mathfrak{g}_{0}=\mathfrak{s o}(k-1,2) \oplus \mathfrak{t}_{0}$ are (2.9), (2.10), (2.12) and (2.13).

\subsection{Global conditions for the decomposition of $\mathfrak{g}_{0}$}

The conditions (2.9), (2.10) and (2.12) we have found on the Killing spinor bilinears in the previous section for $\mathfrak{g}_{0}=\mathfrak{s o}(k-1,2) \oplus \mathfrak{t}_{0}$ can also derived in an elegant way after imposing that the internal space $\mathrm{M}^{d-k}$ is compact without boundary and the fields are smooth. Indeed after setting $\Lambda=\sigma_{+}+\tau_{+}$, one can demonstrate using the KSEs (2.7), (2.8) that

$$
\nabla_{i}\|\Lambda\|^{2}=-\frac{2}{\ell}\left\langle\sigma_{+}, \Gamma_{i} \Gamma_{z} \tau_{+}\right\rangle
$$

and

$$
\nabla^{2}\|\Lambda\|^{2}+2 \partial^{i} \log A \nabla_{i}\|\Lambda\|^{2}=0
$$

Applying the maximum principle, one concludes that $\|\Lambda\|$ is constant which gives in particular (2.12). Then (2.14) implies (2.9). As in the previous section (2.10) can be derived from (2.8) using (2.9). This establishes the assertion.

\section{$3 \quad \mathrm{AdS}_{3}$ in $\mathrm{D}=11$ and type II theories}

\section{$3.1 \quad N=2$}

$\mathrm{AdS}_{3}$ backgrounds preserve an even number of supersymmetries. Therefore the minimal case is that for which a background preserves exactly two supersymmetries. The Killing spinors can be given in terms of either $\sigma_{ \pm}$or $\tau_{ \pm}$spinors. In the former case, we find that the Killing spinors are

$$
\epsilon_{1}=\sigma_{+}, \quad \epsilon_{2}=A \Gamma_{-z} \sigma_{+}+2 \ell^{-1} u \sigma_{+},
$$

where we have set $\sigma_{-}=A \Gamma_{-z} \sigma_{+}$. In terms of $\tau_{ \pm}$the Killing spinors are as in (3.4). Using the results of appendix $\mathrm{C}$, we find that the bilinears are given as

$$
K_{11}=\lambda^{-}, \quad K_{12}=-\lambda^{z}-\ell^{-1} M^{+-}, \quad K_{22}=-2 \lambda^{+}-4 \ell^{-1} M^{z+},
$$

where we have chosen the normalization $2\left\|\sigma_{+}\right\|^{2}=1$ in order to simplify coefficients and $\lambda^{-}, \lambda^{z}, M^{+-}, M^{z+}$ are isometries of $\mathrm{AdS}_{3}$ given in appendix B. Note that all 1-form bilinears have non-vanishing components only along the $\mathrm{AdS}_{3}$ directions. 
The direct computation of spinorial Lie derivatives in appendix $\mathrm{C}$ reveals that

$$
\begin{aligned}
& \mathcal{L}_{K_{11}} \epsilon_{1}=0, \quad \mathcal{L}_{K_{12}} \epsilon_{1}=-\ell^{-1} \epsilon^{1}, \quad \mathcal{L}_{K_{22}} \epsilon_{1}=-2 \ell^{-1} \epsilon_{2}, \\
& \mathcal{L}_{K_{11}} \epsilon_{2}=2 \ell^{-1} \epsilon_{1}, \quad \mathcal{L}_{K_{12}} \epsilon_{2}=\ell^{-1} \epsilon_{2}, \quad \mathcal{L}_{K_{22}} \epsilon_{2}=0 .
\end{aligned}
$$

Using this and the definition of the symmetry superalgebra in (2.1), we find that the non-vanishing commutators are

$$
\begin{aligned}
& \left\{Q_{A}, Q_{B}\right\}=V_{A B}, \quad\left[V_{A B}, Q_{C}\right]=-\ell^{-1}\left(\epsilon_{C A} Q_{B}+\epsilon_{C B} Q_{A}\right), \\
& {\left[V_{A B}, V_{A^{\prime} B^{\prime}}\right]=\ell^{-1}\left(\epsilon_{A A^{\prime}} V_{B B^{\prime}}+\epsilon_{B A^{\prime}} V_{A B^{\prime}}+\epsilon_{A B^{\prime}} V_{B A^{\prime}}+\epsilon_{B B^{\prime}} V_{A A^{\prime}}\right),}
\end{aligned}
$$

where $\epsilon_{A B}, A, B=1,2$, is the Levi-Civita tensor with $\epsilon_{12}=1$. As the Killing spinors have the same form in all 10- and 11-dimensional supergravity theories, the superlgebra of $N=2 \mathrm{AdS}_{3}$ backgrounds in all these theories is (3.3). The Lie algebra of the three Killing vectors $K_{A B}$ is $\mathfrak{s p}(2)=\mathfrak{s l}(2, \mathbb{R})=\mathfrak{s o}(1,2)$ and acts on the two supersymmetry generators with the fundamental representation. This KSA is isomorphic ${ }^{6}$ to $\mathfrak{o s p}(1 \mid 2)$.

\section{$3.2 N=4$}

There are three ways to construct the four Killing spinors of $N=4 \mathrm{AdS}_{3}$ backgrounds in $\mathrm{D}=11$ and type II theories. For the first two options, one can choose the Killing spinors to depend on four linearly independent $\sigma_{ \pm}$spinors or four linearly independent $\tau_{ \pm}$spinors. In the third option, one chooses the first two Killing spinors to depend on $\sigma_{ \pm}$and the remaining two on $\tau_{ \pm}$.

\subsubsection{Left and right superalgebra}

Let us begin with the third possibility where the first two Killing spinors of $N=4 \mathrm{AdS}_{3}$ backgrounds are expressed in terms of $\sigma$ spinors as in (3.1) while the remaining two are expressed in terms of $\tau$ spinors as

$$
\epsilon_{3}=e^{-\frac{z}{\ell}} \tau_{+}-\ell^{-1} r A^{-1} e^{-\frac{z}{\ell}} \Gamma_{-z} \tau_{+}, \quad \epsilon_{4}=A e^{\frac{z}{\ell}} \Gamma_{-z} \tau_{+},
$$

where we have used that if $\tau_{+}$is a Killing spinor then $\tau_{-}=A \Gamma_{-z} \tau_{+}$is also a Killing spinor, and (2.5).

To find the superalgebra in this case, first note that the 1-form bilinears of the $\tau$ type Killing spinors are

$$
K_{33}=\lambda^{-}+2 \ell^{-1} M^{z-}, \quad K_{34}=-\lambda^{z}+\ell^{-1} M^{+-}, \quad K_{44}=-2 \lambda^{+},
$$

where $\lambda^{+}, M^{z-}$ are also isometries of $\mathrm{AdS}_{3}$, see appendix B. So all Killing spinor bilinears lie along the $\mathrm{AdS}_{3}$ subspace directions.

\footnotetext{
${ }^{6}$ We mostly follow the notation [1] for superalgebras. However as different real forms of a superalgebra appear in the analysis, to distinguish between them we have replaced $\mathfrak{o s p}(n, m)$ with $\mathfrak{o s p}(n \mid m)$. If the signature is $(n-2,2)$, we write $\mathfrak{o s p}(n-2,2 \mid m)$. If this is not sufficient to specify the real form, we also use $\mathfrak{g}^{*}$ to denote a different real form from that of $\mathfrak{g}$. In all cases we have investigated the real form of $\mathfrak{g}$ is specified by the real form of $\mathfrak{g}_{0}$ which we present.
} 
It remains to compute the rest of the 1-form bi-linears. Using the orthogonality of $\sigma_{+}$ and $\tau_{+}$spinors (2.10) and (2.9) as well as the expressions for the bilinears in appendix C, one can show that all the remaining bilinears vanish. Then a consequence of the superJacobi identity of the superalgebra is that all the commutators between Killing spinors constructed from $\sigma$ spinors and their bilinears and those constructed from $\tau$ spinors and their bilinears vanish. As a result, the KSA is $\mathfrak{g}=\mathfrak{g}_{L} \oplus \mathfrak{g}_{R}=\mathfrak{o s p}(1 \mid 2) \oplus \mathfrak{o s p}(1 \mid 2)$. Viewing $\mathrm{AdS}_{3}$ locally as a group manifold, $\mathfrak{g}_{L}$ is associated with the left action while $\mathfrak{g}_{R}$ is associated with the right action on $\mathrm{AdS}_{3}$.

\subsubsection{Left or right superalgebra}

Next suppose that all Killing spinors are expressed in terms of four linearly independent $\sigma_{ \pm}$ spinors. In this case, the Killing spinors of $\mathrm{AdS}_{3}$ backgrounds with extended supersymmetry are multiple copies of the Killing spinors (3.1) that appear for the solutions preserving two supersymmetries. Because of this, it is convenient to denote the Killing spinors with a double index as $\epsilon_{A r}$ where $A=1,2$ labels the two spinors in the same multiplet and $r=1, \ldots, N / 2$ denotes the number of multiplets. Using this notation, the Killing spinors of $\mathrm{AdS}_{3}$ backgrounds that preserve four supersymmetries $(N=4)$ can be written as

$$
\epsilon_{1 r}=\sigma_{+}^{r}, \quad \epsilon_{2 r}=A \Gamma_{-z} \sigma_{+}^{r}+2 \ell^{-1} u \sigma_{+}^{r},
$$

where $r=1,2$. We can assume without loss of generality that $\sigma_{+}^{1}$ and $\sigma_{+}^{2}$ are orthogonal. From construction, they have to be linearly independent. As their lengths and inner products are constant and the KSE are linear over the real numbers, they can always be chosen as orthogonal via a Gram-Schmidt process.

An inspection of the results of appendix $\mathrm{C}$ illustrates that the 1-form bilinears of the above Killing spinors can be written as

$$
K_{A r, B s}=K_{A B} \delta_{r s}+\epsilon_{A B} \tilde{K}_{r s},
$$

where $K_{A B}$ are as in (3.2) and $\tilde{K}_{r s}=-\tilde{K}_{s r}=\epsilon_{r s} \tilde{K}$ is a new Killing vector which has nonvanishing components only along the internal space $\mathrm{M}^{d-3}$ directions and depends only on the coordinates of the internal space. After choosing $2\left\langle\sigma_{+}^{r}, \sigma_{+}^{s}\right\rangle=\delta_{r s}$, the anti-commutator of the odd generators can be written as in

$$
\left\{Q_{A r}, Q_{B s}\right\}=V_{A B} \delta_{r s}+\epsilon_{r s} \epsilon_{A B} \tilde{V},
$$

where we have set $\tilde{V}_{r s}=\epsilon_{r s} \tilde{V}$.

All the commutators $\left[V_{A B}, Q_{C r}\right]$ can be read from the results of appendix C. It remains to determine the commutator $\left[\tilde{V}, Q_{A r}\right]$. As we do not have additional information on the geometry of the internal space $\mathrm{M}^{d-3}$, this commutator cannot be computed explicitly. Instead, we shall utilize the closure of the KSA. For this first observe that $\tilde{V}=\left\{Q_{11}, Q_{22}\right\}=$ $-\left\{Q_{12}, Q_{21}\right\}$. Thus for every choice of $Q_{A r}$ there is another odd generator $Q_{A^{\prime} r^{\prime}}$ with $A \neq A^{\prime}$ and $r \neq r^{\prime}$ such that $\left\{Q_{A r}, Q_{A^{\prime} r^{\prime}}\right\} \propto \tilde{V}$. Then the super-Jacobi identities imply that

$$
\begin{aligned}
{\left[\tilde{V}, Q_{A r}\right] } & \propto\left[\left\{Q_{A r}, Q_{A^{\prime} r^{\prime}}\right\}, Q_{A r}\right]=-\frac{1}{2}\left[\left\{Q_{A r}, Q_{A r}\right\}, Q_{A^{\prime} r^{\prime}}\right]=-\frac{1}{2}\left[V_{A A}, Q_{A^{\prime} r^{\prime}}\right] \\
& =\ell^{-1} \epsilon_{A^{\prime} A} Q_{A r^{\prime}} .
\end{aligned}
$$


Using this and after a brief computation, one can verify that the superalgebra is

$$
\begin{aligned}
\left\{Q_{A r}, Q_{B s}\right\} & =V_{A B} \delta_{r s}+\epsilon_{r s} \epsilon_{A B} \tilde{V} \\
{\left[V_{A B}, Q_{C r}\right] } & =-\ell^{-1}\left(\epsilon_{C A} Q_{B r}+\epsilon_{C B} Q_{A r}\right), \\
{\left[\tilde{V}, Q_{A r}\right] } & =-\ell^{-1} \epsilon_{r}^{s} Q_{A s},
\end{aligned}
$$

which is isomorphic to $\mathfrak{o s p}(2 \mid 2)$. The analysis for the case in which all the Killing spinors depend on $\tau_{ \pm}$spinors follows in the same way.

Note that as there is some freedom to choose $\sigma_{+}^{2}$, it is not a priori obvious that there is a non-vanishing 1-form bilinear $\tilde{K}_{r s}$ associated to the generator $\tilde{V}_{r s}$. However if $\tilde{K}_{r s}$ is chosen to vanish and so the superalgebra does not have a $\tilde{V}_{r s}$ generator, the super-Jacobi identities of three $Q_{A r}$ generators are not satisfied. Therefore consistency of the KSA requires the presence of the $\tilde{V}_{r s}$ generator.

\subsection{Some structure theory}

\subsubsection{Structure constants of KSAs}

To proceed further for $N>4$ let us suppose that all Killing spinors $\epsilon_{A r}$ are constructed from linearly independent $\sigma_{ \pm}^{r}$ spinors. Then as we shall explain below the (anti)-commutators of the superagebra can be written as

$$
\begin{aligned}
\left\{Q_{A r}, Q_{B s}\right\} & =\delta_{r s} V_{A B}+\epsilon_{A B} \tilde{V}_{r s}, \\
{\left[V_{A B}, Q_{C r}\right] } & =-\ell^{-1}\left(\epsilon_{C A} Q_{B r}+\epsilon_{C B} Q_{A r}\right), \\
{\left[V_{A B}, V_{A^{\prime} B^{\prime}}\right] } & =\ell^{-1}\left(\epsilon_{A A^{\prime}} V_{B B^{\prime}}+\epsilon_{B A^{\prime}} V_{A B^{\prime}}+\epsilon_{A B^{\prime}} V_{B A^{\prime}}+\epsilon_{B B^{\prime}} V_{A A^{\prime}}\right), \\
{\left[\tilde{V}_{r s}, Q_{A t}\right] } & =-\ell^{-1}\left(\delta_{t r} Q_{A s}-\delta_{t s} Q_{A r}\right)+\ell^{-1} \alpha_{r s t}{ }^{p} Q_{A p},
\end{aligned}
$$

where as it will be explained below $\alpha$ is a constant 4 -form. The first anti-commutator follows from the results of appendix $\mathrm{C}$ where we have normalized the spinors as $2\left\langle\sigma_{+}^{r}, \sigma_{+}^{s}\right\rangle=\delta_{r s}$. The bosonic generators $V_{A B}$ are associated with the left $\mathrm{AdS}_{3}$ isometries, and the $\tilde{V}_{r s}$ are associated with isometries of the internal space. However note that $\tilde{V}_{r s}$ are not necessarily linearly independent. The second and third commutators follow from a direct computation presented in appendices $\mathrm{B}$ and $\mathrm{C}$ as the dependence of the Killing spinors and $K_{A B}$ on $\mathrm{AdS}_{3}$ coordinates is known. Then the fourth commutator can be restricted by requiring consistency with the super-Jacobi identities.

To justify the $\left[\tilde{V}_{r s}, Q_{A t}\right]$ commutator observe that if either $t=r$ or $t=s$, the commutator follows from the results established in the $N=4$ case. Now suppose that $t \neq r, s$ and consider $\mathcal{L}_{\tilde{K}_{r s}} \epsilon_{A t}$. As we are investigating backgrounds preserving strictly $\mathrm{N}$ supersymmetries there must be constants $\alpha$ and $\tilde{\alpha}$ such that

$$
\mathcal{L}_{\tilde{K}_{r s}} \epsilon_{A t}=\alpha_{A r s t}{ }^{\ell} \epsilon_{A \ell}+\epsilon_{A}{ }^{B} \tilde{\alpha}_{A r s t}{ }^{\ell} \epsilon_{B \ell}, \quad t \neq r, s,
$$

where $\alpha_{A r s t}{ }^{\ell}=-\alpha_{A s r t}{ }^{\ell}, \tilde{\alpha}_{A r s t}{ }^{\ell}=-\tilde{\alpha}_{A s r t}^{\ell}$ and there is no summation over the index A. As $\tilde{K}_{r s}$ are along the internal manifold, the spinorial Lie derivative preserves the dependence of the Killing spinors on the $\mathrm{AdS}_{3}$ coordinates. Therefore $\tilde{\alpha}=0$. In addition, $\alpha_{1 r s t}^{\ell}=\alpha_{2 r s t}^{\ell}=\alpha_{r s t}^{\ell}$ as it can be seen after using the super-Jacobi identity of the 
generators $\tilde{V}_{r s}, V_{B B}$ and $Q_{A t}, A \neq B$. Furthermore, the super-Jacobi identity of the generators $Q_{A r}, Q_{B s}$ and $Q_{A t}$, for $A \neq B$, implies that $\alpha_{r s t}^{\ell}=\alpha_{s t r}^{\ell}$. This together with $\alpha_{r s t}^{\ell}=-\alpha_{\text {srt }}^{\ell}$ gives

$$
\alpha_{r s t}^{\ell}=\alpha_{[r s t]}^{\ell} .
$$

Furthermore as $\epsilon_{1 t}=\sigma_{+}^{t}$ and $\mathcal{L}_{\tilde{K}_{r s}}\left\langle\sigma_{+}^{t}, \sigma_{+}^{\ell}\right\rangle=0$, we find that

$$
\alpha_{r s t \ell}=-\alpha_{r s \ell t},
$$

where we have lowered the index with $\delta_{r s}$. Combining (3.13) and (3.14), we deduce that

$$
\alpha_{r s t \ell}=\alpha_{[r s t \ell]},
$$

and so $\alpha$ is a 4 -form.

The identification of KSAs of $\mathrm{AdS}_{3}$ backgrounds for $N>4$ depends crucially on determining the form $\alpha$. One way to do this is to observe that the outer automorphisms of KSA include the action of $\mathrm{SO}(N / 2)$ on $Q_{A r}$. With this action $\alpha$ transforms as a 4 -form. As a result it suffices to consider representatives of the orbits of $\mathfrak{s o}(N / 2)$ on the space of 4-forms. This consideration is sufficient to identify all the KSAs for $N \leq 12$ and it is explored in appendix D. However to find all KSAs, we investigate the structure of these KSAs further.

\subsubsection{The $\mathrm{AdS}_{3}$ KSAs are direct sums of Left and Right KSAs}

Before we proceed further with the investigation of the KSA, let us consider the case where some of the Killing spinors are constructed from $\sigma$ spinors and some others from $\tau$ spinors. A straightforward application of the computation presented in appendix $\mathrm{C}$ reveals that the superalgebra $\mathfrak{g}(\sigma)$ associated to the $\sigma$ type of Killing spinors and the superalgebra $\mathfrak{g}(\tau)$ associated to the $\tau$ type of Killing spinors commute, and so we can set $\mathfrak{g}_{L}=\mathfrak{g}(\sigma)$ and $\mathfrak{g}_{R}=\mathfrak{g}(\tau) ; \mathfrak{g}=\mathfrak{g}_{L} \oplus \mathfrak{g}_{R}$. To see this observe that all mixed $\sigma$ and $\tau$ 1-form bilinears vanish. This implies that the odd $\sigma$ type generators anti-commute with the odd $\tau$ type generators. Furthermore the commutator of $\sigma$ type even generators associated to isometries on $\mathrm{AdS}_{3}$ with $\tau$ type odd generators vanishes, and vice versa. This can be seen from the spinorial Lie derivatives in appendix $\mathrm{C}$.

It remains to demonstrate that the commutators of $\sigma(\tau)$ type even generators associated to isometries on the internal space with $\tau(\sigma)$ type odd generators vanish as well. First observe that Killing vectors along the internal space preserve the functional dependence of Killing spinors on the $\mathrm{AdS}_{3}$ coordinates. As $\sigma$ and $\tau$ Killing spinors have different such dependence it follows that they cannot be rotated to each other under such spinorial Lie derivatives. Then upon using super-Jacobi identities and the fact that all mixed bilinears vanish, one can show that the commutator of a $\sigma(\tau)$ internal even generator with any $\tau$ $(\sigma)$ odd generator vanishes. This establishes the result.

A consequence of $\mathfrak{g}=\mathfrak{g}_{L} \oplus \mathfrak{g}_{R}$ is that it suffices to investigate the KSAs associated with only $\sigma$ type Killing spinors. Then $\mathfrak{g}$ can be easily found as the construction of $\mathfrak{g}_{R}$ superalgebras is identical to the one that follows for $\mathfrak{g}_{L}$. Therefore the list of $\mathfrak{g}_{R}$ superalgebras that can occur is the same as that for $\mathfrak{g}_{L}$ superalgebras. Though for a given background $\mathfrak{g}_{L}$ may not be isomorphic to $\mathfrak{g}_{R}$. 


\subsubsection{Structure theorems}

Let $\mathfrak{g}=\mathfrak{g}_{L}$ be the super-algebra of $\mathrm{AdS}_{3}$ backgrounds preserving $N$ supersymmetries. Decompose $\mathfrak{g}_{0}=\mathfrak{s p}(2) \oplus \mathfrak{t}_{0}$, where $\mathfrak{t}_{0}=\operatorname{Span}\left(\tilde{V}_{r s}\right)$. It is clear that $\mathfrak{g}_{1}=\mathbb{R}^{2} \otimes \mathbb{R}^{\frac{N}{2}}$ and the action of $\mathfrak{t}_{0}$ preserves the Euclidean inner product on $\mathbb{R}^{\frac{N}{2}}$. As a result $\mathfrak{t}_{0} \subseteq \mathfrak{s o}(N / 2)$. We shall show that $\mathfrak{t}_{0}$ is associated with a subgroup of $\mathrm{SO}(N / 2)$ which acts transitively on the $S^{\frac{N}{2}-1}$ sphere in $\mathbb{R}^{\frac{N}{2}}$. To demonstrate this we shall first show the following.

Proposition. Given $u, w \in \mathbb{R}^{\frac{N}{2}}$ and $u, w$ linearly independent, then $\tilde{V}_{u \times w} \equiv u^{r} w^{s} \tilde{V}_{r s}$ cannot vanish.

Proof. Suppose that $\tilde{V}_{u \times w}=0$. In such a case it follows from (3.11) that

$$
\left\{u \cdot Q_{A}, w \cdot Q_{B}\right\}=u \cdot w \quad V_{A B},
$$

where $u \cdot w$ is the Euclidean inner product in $\mathbb{R}^{\frac{N}{2}}$ and $u \cdot Q_{A}=u^{r} Q_{A r}$. Then upon using the super-Jacobi identity

$$
\begin{aligned}
u^{2} \ell^{-1} \epsilon_{A B} w \cdot Q_{B} & =u^{2}\left[V_{A B}, w \cdot Q_{B}\right]=\left[\left\{u \cdot Q_{A}, u \cdot Q_{B}\right\}, w \cdot Q_{B}\right] \\
& =-\left[\left\{w \cdot Q_{B}, u \cdot Q_{A}\right\}, u \cdot Q_{B}\right]-\left[\left\{u \cdot Q_{B}, w \cdot Q_{B}\right\}, u \cdot Q_{A}\right] \\
& =-u \cdot w\left(\left[V_{A B}, u \cdot Q_{B}\right]+\left[V_{B B}, u \cdot Q_{A}\right]\right)=\ell^{-1} \epsilon_{A B} u \cdot w u \cdot Q_{B},
\end{aligned}
$$

which is satisfied iff $u$ and $w$ are linearly dependent as $\operatorname{dim} \mathfrak{g}_{1}=N$. This a contradiction and so $\tilde{V}_{u \times w} \neq 0$.

Proposition. The Lie algebra $\mathfrak{t}_{0}$ is associated with a subgroup $H_{0}$ of $\mathrm{SO}(N / 2)$ that acts transitively on the sphere $S^{\frac{N}{2}-1} \subset \mathbb{R}^{\frac{N}{2}}$.

Proof. It suffices to show that given two linearly independent vectors $u, w \in \mathbb{R}^{\frac{N}{2}}$, there is element $R(u, w) \in \mathfrak{t}_{0}$ such that $R(u, w)$ generates $\mathrm{SO}(2)$ rotations on the 2-plane spanned by $u$ and $w$ in $\mathbb{R}^{\frac{N}{2}}$. As the $\mathrm{SO}(2)$ rotations act transitively on all directions in the 2-plane spanned by $u$ and $w$, it follows that there is an element in $H_{0}$ which rotates the direction defined by the vector $u$ onto that of the vector $w$.

For this set $R(u, w)=\tilde{V}_{u \times w}$ and observe that

$$
\left[\tilde{V}_{u \times w}, p \cdot Q_{A}\right]=-\ell^{-1}\left(p \cdot u w \cdot Q_{A}-p \cdot w u \cdot Q_{A}\right),
$$

for any $p$ that lies in the 2-plane spanned by $u$ and $w$. So indeed $\tilde{V}_{u \times w}$ acts as an infinitesimal orthogonal rotation on the 2-plane spanned by $u$ and $w$. As this can be done for any $u, w \in \mathbb{R}^{\frac{N}{2}}$, it follows that $H_{0}$ acts transitively on $S^{\frac{N}{2}-1} \subset \mathbb{R}^{\frac{N}{2}}$.

Proposition. The representation of Lie algebra $\mathfrak{t}_{0}$ on $\mathfrak{g}_{1}$ leaves invariant the 4 -form $\alpha$.

Proof. For this write

$$
\left[\tilde{V}_{r s}, Q_{A t}\right]=D\left(\tilde{V}_{r s}\right)_{t}^{\ell} Q_{A \ell} .
$$

It suffices to show that

$$
D\left(\tilde{V}_{r s}\right)_{\left[t_{1}\right.}{ }^{\ell} \alpha_{\left.t_{2} t_{3} t_{4}\right] \ell}=0 .
$$


Lie Algebras of Groups Acting Transitively on Spheres

\begin{tabular}{|c|c|c|}
\hline Algebra & Sphere & $N / 2$ \\
\hline $\mathfrak{s o}(n)$ & $S^{n-1}$ & $n$ \\
\hline $\mathfrak{u}(n)$ & $S^{2 n-1}$ & $2 n$ \\
\hline $\mathfrak{s u}(n)$ & $S^{2 n-1}$ & $2 n$ \\
\hline $\mathfrak{s p}(n) \oplus \mathfrak{s p}^{*}(1)$ & $S^{4 n-1}$ & $4 n$ \\
\hline $\mathfrak{s p}^{*}(n) \oplus \mathfrak{u}(1)$ & $S^{4 n-1}$ & $4 n$ \\
\hline $\mathfrak{s p}(n)$ & $S^{4 n-1}$ & $4 n$ \\
\hline $\mathfrak{g}_{2}$ & $S^{6}$ & 7 \\
\hline $\mathfrak{s p i n}(7)$ & $S^{7}$ & 8 \\
\hline $\mathfrak{s p i n}(9)$ & $S^{15}$ & 16 \\
\hline
\end{tabular}

Table 1. $\mathfrak{s p}^{*}(n)$ is the compact symplectic algebra with (real) dimension $n(2 n+1)$ and a real form of $\mathfrak{s p}(2 n)$.

First using the super-Jacobi identities, one can establish that

$$
\begin{aligned}
{\left[\tilde{V}_{r s}, \tilde{V}_{r^{\prime} s^{\prime}}\right]=} & -\ell^{-1}\left(\delta_{r r^{\prime}} \tilde{V}_{s s^{\prime}}-\delta_{s r^{\prime}} \tilde{V}_{r s^{\prime}}-\delta_{r s^{\prime}} \tilde{V}_{s r^{\prime}}+\delta_{s s^{\prime}} \tilde{V}_{r r^{\prime}}\right. \\
& \left.-\alpha_{r s r^{\prime}}{ }^{t} \tilde{V}_{t s^{\prime}}+\alpha_{r s s^{\prime}}{ }^{t} \tilde{V}_{t r^{\prime}}\right) .
\end{aligned}
$$

As this bracket is skew-symmetric in the interchange of the pair $\tilde{V}_{r s}$ and $\tilde{V}_{r^{\prime} s^{\prime}}$, one obtains the identity

$$
\alpha_{r s r^{\prime}}{ }^{t} \tilde{V}_{t s^{\prime}}-\alpha_{r s s^{\prime}}{ }^{t} \tilde{V}_{t r^{\prime}}+\alpha_{r^{\prime} s^{\prime}{ }^{t}}{ }^{t} \tilde{V}_{t s}-\alpha_{r^{\prime} s^{\prime} s}{ }^{t} \tilde{V}_{t r}=0
$$

Taking the commutator with $Q_{A p}$, one arrives at (3.20).

The results we have obtained above can be summarized as follows.

Theorem. The necessary conditions for a superalgebra $\mathfrak{g}$ to be the KSA of $\mathrm{AdS}_{3}$ backgrounds are that $\mathfrak{g}_{0}=\mathfrak{s p}(2) \oplus \mathfrak{t}_{0}$ and that $\mathfrak{g}_{1}=\mathbb{R}^{2} \otimes \mathbb{R}^{\frac{N}{2}}$, where $\mathfrak{t}_{0}$ is the Lie algebra of a group acting transitively on $S^{\frac{N}{2}-1} \subset \mathbb{R}^{\frac{N}{2}}$. Furthermore, the representation of the Lie algebra $\mathfrak{t}_{0}$ in $\mathfrak{g}_{1}$ leaves the 4 -form $\alpha$ invariant.

The groups that act effectively and transitively on spheres have been classified in [10] and have been listed in table 1 . This classification also specifies the representation of the group that acts transitively on the vector space $\mathbb{R}^{n}$ in which $S^{n-1}$ is embedded. This is essential for finding the $\mathrm{AdS}_{3} \mathrm{KSAs}$ as we shall explain below.

To identify $\mathfrak{t}_{0}$ with the Lie algebras of the groups listed in table 1 , it remains to find the conditions for $\mathfrak{t}_{0}$ to act effectively on $\mathfrak{g}_{1}$. For this define the subalgebra $\mathfrak{c} \subset \mathfrak{t}_{0}$ such that $\left[\mathfrak{c}, \mathfrak{g}_{1}\right]=0$ or equivalently

$$
\mathfrak{c}=\left\{u^{r s} \tilde{V}_{r s} \in \mathfrak{t}_{0} \mid 2 u^{r s}-u^{p q} \alpha_{p q}^{r s}=0\right\} .
$$

Observe that $\mathfrak{c}$ is a commutative ideal ${ }^{7}$ of $\mathfrak{g}, \mathfrak{g}_{0} / \mathfrak{c}=\mathfrak{s p}(2) \oplus \mathfrak{t}_{0} / \mathfrak{c}$ and $\mathfrak{t}_{0} / \mathfrak{c}$ acts effectively on $\mathfrak{g}_{1}$. As $\mathfrak{t}_{0} / \mathfrak{c}$ also acts transitively on the spheres, it can be identified with the Lie algebra

\footnotetext{
${ }^{7}$ Super-Jacobi identities put additional restrictions on $\mathfrak{c}$ which may lead to the vanishing of all its elements.
} 
of the groups listed in table 1. Significantly, the representation of $\mathfrak{t}_{0} / \mathfrak{c}$ on $\mathfrak{g}_{1}$ is determined from that of the groups listed in table 1 on $\mathbb{R}^{\frac{N}{2}}$ in which the sphere $S^{\frac{N}{2}-1}$ is embedded. If $\mathfrak{c}$ is non-empty, then $\mathfrak{g}$ is not simple. This is the reason that non-simple superalgebras can occur as KSAs for $\mathrm{AdS}_{3}$ backgrounds ${ }^{8}$

It should be noted that the KSA of $\mathrm{AdS}_{3}$ backgrounds admits a consistent, supersymmetric and invariant inner product ${ }^{9}$ given by

$$
\begin{aligned}
\left\langle Q_{A r}, Q_{B s}\right\rangle & =\epsilon_{A B} \delta_{r s}, \quad\left\langle V_{A B}, V_{A^{\prime} B^{\prime}}\right\rangle=-\ell^{-1} \epsilon_{A A^{\prime}} \epsilon_{B B^{\prime}}-\ell^{-1} \epsilon_{B A^{\prime}} \epsilon_{A B^{\prime}} \\
\left\langle\tilde{V}_{r s}, \tilde{V}_{r^{\prime} s^{\prime}}\right\rangle & =\ell^{-1}\left[\delta_{r r^{\prime}} \delta_{s s^{\prime}}-\delta_{s r^{\prime}} \delta_{r s^{\prime}}-\alpha_{r s r^{\prime} s^{\prime}}\right] .
\end{aligned}
$$

Observe that if $\mathfrak{c} \neq \emptyset$, this inner product is degenerate.

\subsection{KSAs from $\mathfrak{s o}(n)$ acting transitively on $S^{n-1}$}

\subsubsection{Generic case $N=N_{\sigma}=2 n$}

If $N \neq 8$, then $\mathfrak{s o}(n)$ does not admit an invariant 4 -form in the fundamental $n$-dimensional representation and thus $\alpha=0$. Then it is straightforward to observe that the KSA is isomorphic to $\mathfrak{o} \mathfrak{s p}(n \mid 2)$. The same applies in the $N=8$ case provided we choose $\alpha=0$. As there are no maximally supersymmetric $\mathrm{AdS}_{3}$ backgrounds $n<16$. The algebra of isometries of the internal space is $\mathfrak{s o}(n)$.

\subsection{2 $N=N_{\sigma}=8$}

As $\alpha$ is a 4 -form (3.12) in a 4-dimensional space it is proportional to the volume form $\epsilon$. So we write

$$
\alpha_{r s t \ell}=\hat{\alpha} \epsilon_{r s t \ell}
$$

for some constant $\hat{\alpha}$. Using this, the non-vanishing (anti-)commutators (3.11) of KSA are

$$
\begin{aligned}
\left\{Q_{A r}, Q_{B s}\right\}= & V_{A B} \delta_{r s}+\epsilon_{A B} \tilde{V}_{r s}, \\
{\left[V_{A B}, Q_{C r}\right]=} & -\ell^{-1}\left(\epsilon_{C A} Q_{B r}+\epsilon_{C B} Q_{A r}\right), \\
{\left[\tilde{V}_{r s}, Q_{A t}\right]=} & -\ell^{-1}\left(\delta_{t r} Q_{A s}-\delta_{t s} Q_{A r}\right)+\ell^{-1} \hat{\alpha} \epsilon_{r s t}{ }^{p} Q_{A p}, \\
{\left[\tilde{V}_{r s}, \tilde{V}_{r^{\prime} s^{\prime}}\right]=} & -\ell^{-1}\left(\delta_{r r^{\prime}} \tilde{V}_{s s^{\prime}}-\delta_{s r^{\prime}} \tilde{V}_{r s^{\prime}}-\delta_{r s^{\prime}} \tilde{V}_{s r^{\prime}}+\delta_{s s^{\prime}} \tilde{V}_{r r^{\prime}}\right. \\
& \left.-\hat{\alpha} \epsilon_{r s r^{\prime}}{ }^{t} \tilde{V}_{t s^{\prime}}+\hat{\alpha} \epsilon_{r s s^{\prime}}{ }^{t} \tilde{V}_{t r^{\prime}}\right),
\end{aligned}
$$

where we have neglected the commutators of the $V_{A B}$ already given in (3.3). This algebra is isomorphic to a real form of the $\mathfrak{D}(2,1 ; \alpha)$ superalgebra with $\alpha=\frac{\hat{\alpha}+1}{1-\hat{\alpha}}$ and $\hat{\alpha} \neq 0,1,-1$. The isometry algebra of the internal space is $\mathfrak{s o}(3) \oplus \mathfrak{s o}(3)$. If $\hat{\alpha}=0$, this superalgebra is isomorphic to $\mathfrak{o s p}(4 \mid 2)$ as expected.

It remains to investigate the cases for which $\hat{\alpha}$ is either 1 or -1 . For this write $\tilde{V}_{r s}=\tilde{V}_{P}^{+}\left(\omega_{P}^{(+)}\right)_{r s}+\tilde{V}_{P}^{-}\left(\omega_{P}^{(-)}\right)_{r s}$ where $\omega^{( \pm)}$are orthonormal bases in the space of self-dual

\footnotetext{
${ }^{8}$ To our knowledge there does not exist an example of an $\mathrm{AdS}_{3}$ solution with a non-simple KSA. So such solutions may not exist. However, they cannot be ruled out within our framework as their exclusion requires a more detailed description of the geometry of the internal space.

${ }^{9}$ For the definition see $[1]$.
} 
and anti-self dual two forms $\omega_{P r s}^{( \pm)} \omega_{Q}^{( \pm) r s}=4 \delta_{P Q}$. Then $\left[\tilde{V}_{r s}, Q_{A t}\right]$ can be rewritten as

$$
\begin{aligned}
& {\left[\tilde{V}_{P}^{+}, Q_{A t}\right]=\frac{1}{2} \ell^{-1}(\hat{\alpha}-1)\left(\omega_{P}^{(+)}\right) t^{s} Q_{A s},} \\
& {\left[\tilde{V}_{P}^{-}, Q_{A t}\right]=-\frac{1}{2} \ell^{-1}(\hat{\alpha}+1)\left(\omega_{P}^{(-)}\right)_{t}^{s} Q_{A s} .}
\end{aligned}
$$

If either $\tilde{V}^{+}$or $\tilde{V}^{-}$vanishes for either $\hat{\alpha}=1$ or $\hat{\alpha}=-1$, respectively, the superalgebra is a real form of $\mathfrak{s l}(2 \mid 2) / 1_{4 \times 4}$. The isometry algebra of the internal space is $\mathfrak{s o}(3)$.

Next consider the possibility that both $\tilde{V}^{+}$and $\tilde{V}^{-}$do not vanish and either $\hat{\alpha}=1$ or $\hat{\alpha}=-1$. In such a case, it can be seen that $\tilde{V}^{+}$for $\hat{\alpha}=1$ or $\tilde{V}^{-}$for $\hat{\alpha}=-1$ become central and they are allowed to be non-vanishing as they do not appear on the right-handside of $\mathfrak{s o}(3)$ commutators generated by either $\tilde{V}^{-}$or $\tilde{V}^{+}$, respectively. We shall denote the resulting superalgebra with $\mathfrak{c s l}(2 \mid 2 ; 3) / 1_{4 \times 4}$ where the last numerical entry denotes the maximal number of central generators. This is not a (semi-)simple superalgebra. It is not apparent that such a superalgebra arises as a possibility in actual solutions. However it cannot be ruled out on the grounds of symmetry and the geometric assumptions we have made.

\subsection{KSAs from $\mathfrak{u}(n)$ acting transitively on $S^{2 n-1}$}

In this case $N=N_{\sigma}=4 n$. Define an embedding of $\mathfrak{u}(n)$ into $\mathfrak{s o}(2 n)$ by choosing a complex structure $I$ in $\mathbb{R}^{2 n}$ compatible with the Euclidean metric and with associated Hermitian form $\omega, \omega_{r s}=\delta_{r t} I_{s}^{t}$. The 4 -form $\alpha$ can be chosen as

$$
\alpha=\hat{\alpha} \omega \wedge \omega
$$

where $\hat{\alpha}$ is a constant. The subalgebra $\mathfrak{t}_{0}$ decomposes as

$$
\mathfrak{t}_{0}=\mathfrak{t}_{0}^{(1,1)} \oplus \mathfrak{t}_{0}^{(2,0)+(0,2)}
$$

where $\mathfrak{t}_{0}^{(2,0)+(0,2)}$ and $\mathfrak{t}_{0}^{(1,1)}$ are the spaces of $(2,0)$ - and $(0,2)$-forms and (1,1)-forms with respect to $I$, respectively. So $\mathfrak{t}_{0}^{(1,1)}=\mathfrak{u}(n)$. The projectors are given by

$$
\left(P^{(1,1)}\right)_{r s}^{r^{\prime} s^{\prime}}=\frac{1}{2}\left(\delta^{r^{\prime}}{ }_{[r} \delta^{s^{\prime}}{ }_{s]}+I^{r^{\prime}}{ }_{[r}{I^{s^{\prime}}}_{s]}\right), \quad\left(P^{(2,0)+(0,2)}\right)_{r s}^{r^{\prime} s^{\prime}}=\frac{1}{2}\left(\delta^{r^{\prime}}{ }_{[r} \delta^{s^{\prime}}{ }_{s]}-I_{[r}^{r^{\prime}} I_{s]}^{s^{\prime}}\right) .
$$

If both $\tilde{V}^{(1,1)}$ and $\tilde{V}^{(2,0)+(0,2)}$ act effectively on the $Q$ 's, then consistency requires that $\mathfrak{s o}(2 n)$ acts with the fundamental representation on the $Q$ 's and the KSA must be isomorphic to $\mathfrak{o} \mathfrak{s p}(2 n \mid 2)$. Alternatively only the subalgebra $\mathfrak{u}(n)$ acts effectively on the $Q$ 's. Imposing this by requiring that the elements of $\mathfrak{t}_{0}^{(2,0)+(0,2)}$ commute with the $Q$ 's gives that

$$
\hat{\alpha}=\frac{1}{2}
$$

Furthermore one finds that

$$
\left[\tilde{V}_{r s}^{(1,1)}, Q_{A t}\right]=-\ell^{-1}\left(\delta_{t r} Q_{A s}-\delta_{t s} Q_{A r}+\omega_{t r} I^{p}{ }_{s} Q_{A p}-\omega_{t s} I_{r}{ }_{r} Q_{A p}+\omega_{r s} I^{p}{ }_{t} Q_{A p}\right)
$$


Observe that although (3.32) does not give the standard action of $\mathfrak{u}(n)$ on $\mathbb{C}^{n}$ because of the last term in the commutator, this can be achieved after the change of basis

$$
\tilde{V}_{r s}^{(1,1)} \rightarrow \tilde{V}_{r s}^{(1,1)}+\frac{1}{2(2-n)} \omega_{r s} \omega^{p q} \tilde{V}_{p q}^{(1,1)}, \quad n \neq 2
$$

Factoring with the ideal generated by $\mathfrak{c}=\tilde{V}^{(2,0)+(0,2)}$, the resulting KSA is $\mathfrak{s l}(n \mid 2)$.

A consequence of (3.32) is that

$$
\left[\omega^{r s} \tilde{V}_{r s}^{(1,1)}, Q_{A t}\right]=2 \ell^{-1}(2-n) I^{p}{ }_{t} Q_{A p},
$$

ie the $\omega$-trace of the $\tilde{V}^{(1,1)}$ has a non-trivial action on the $Q$ 's for $n \neq 2$. As a result for $n \neq 2$, there is no a KSA which is associated with $\mathfrak{s u}(n)$ Lie algebra ${ }^{10}$ in table 1 as this would require that $\omega^{r s} \tilde{V}_{r s}^{(1,1)}$ commutes with the $Q$ 's. However for $n=2$, one can further factor with $\omega^{p q} \tilde{V}_{p q}^{(1,1)}$ yielding the superalgebra $\mathfrak{s l}(2 \mid 2) / 1_{4 \times 4}$.

\subsection{KSAs from $\mathfrak{g}_{2}$ and $\mathfrak{s p i n}(7)$}

The argument required to identify the KSA in these two cases proceeds as in the $\mathfrak{u}(n)$ case. In the $\mathfrak{g}_{2} \subset \mathfrak{s o}(7)$ case for which $N=N_{\sigma}=14$, the invariant 4-form can be chosen as $\alpha=\hat{\alpha} \phi$ where $\hat{\alpha}$ is a constant and $\phi$ is the fundamental invariant 4 -form of $\mathfrak{g}_{2}$. Moreover $\mathfrak{t}_{0}=\mathfrak{t}_{0}^{\mathbf{1 4}} \oplus \mathfrak{t}_{0}^{\mathbf{7}}$, where $\mathfrak{t}_{0}^{\mathbf{1 4}}=\mathfrak{g}_{2}$. The projectors are

$$
\left(P_{\mathbf{1 4}}\right)_{r s}^{r^{\prime} s^{\prime}}=\frac{2}{3}\left(\delta^{r^{\prime}}{ }_{[r} \delta^{s^{\prime}}{ }_{s]}+\frac{1}{4} \phi_{r s}{ }^{r^{\prime} s^{\prime}}\right), \quad\left(P_{\mathbf{7}}\right)_{r s}^{r^{\prime} s^{\prime}}=\frac{1}{3}\left(\delta^{r^{\prime}}{ }_{[r} \delta_{s]}^{s^{\prime}}-\frac{1}{2} \phi_{r s}{ }^{r^{\prime} s^{\prime}}\right)
$$

where $r, s, r^{\prime}, s^{\prime}=1, \ldots, 7$. It is clear from this that $P_{\mathbf{7}} \tilde{V} \in \mathfrak{t}_{0}^{7}$ commute with the $Q$ 's provided that $\hat{\alpha}=-1 / 2$. The superalgebra $\mathfrak{g} / \mathfrak{t}_{0}^{7}$ is isomorphic to $\mathfrak{g}(3)$ and $\mathfrak{c}=\mathfrak{t}_{0}^{7} . P_{\mathbf{7}} \tilde{V}$ must vanish because if they do not, these generators appear as central extensions of $\mathfrak{g}_{2}$ which is simple and so it does not admit such an extension.

In the $\mathfrak{s p i n}(7) \subset \mathfrak{s o}(8)$ case for which $N=N_{\sigma}=16$, the invariant 4 -form is the fundamental, invariant, self-dual form $\psi$. So we choose $\alpha=\hat{\alpha} \psi$. Furthermore $\mathfrak{t}_{0}=\mathfrak{t}_{0}^{\mathbf{2 1}} \oplus \mathfrak{t}_{0}^{\mathbf{7}}$ with $\mathfrak{t}_{0}^{\mathbf{2 1}}=\mathfrak{s p i n}(7)$. The projectors are

$$
\left(P_{\mathbf{2 1}}\right)_{r s}^{r^{\prime} s^{\prime}}=\frac{1}{4}\left(3 \delta^{r^{\prime}}{ }_{[r} \delta^{s^{\prime}}{ }_{s]}+\frac{1}{2} \psi_{r s}{ }^{r^{\prime} s^{\prime}}\right), \quad\left(P_{\mathbf{7}}\right)_{r s}^{r^{\prime} s^{\prime}}=\frac{1}{4}\left(\delta^{r^{\prime}}{ }_{[r} \delta^{s^{\prime}}{ }_{s]}-\frac{1}{2} \psi_{r s}{ }^{r^{\prime} s^{\prime}}\right)
$$

where now $r, s, r^{\prime}, s^{\prime}=1, \ldots, 8$. For $P_{\mathbf{7}} \tilde{V} \in \mathfrak{t}_{0}^{7}$ to commute with the $Q$ 's, one has to set $\hat{\alpha}=-1 / 3$. The superalgebra $\mathfrak{g} / \mathfrak{t}_{0}^{7}$ is isomorphic to $\mathfrak{f}(4)$ and $\mathfrak{c}=\mathfrak{t}_{0}^{7}$. The super-Jacobi identity of the even generators requires that one has to set $\mathfrak{t}_{0}^{7}$ to zero as $\mathfrak{s p i n}(7)$ does not admit central extensions.

\footnotetext{
${ }^{10}$ There is an exception to this for $n=4$ where additional invariant 4 -forms exist associated with the holomorphic $(4,0)$-form. Consideration of these leads to the $\mathfrak{s p i n}(7)$ case that we shall investigate below.
} 


\subsection{KSAs from $\mathfrak{s p}^{*}(n) \oplus \mathfrak{s p}^{*}(1)$ and the remaining cases}

3.7.1 $\mathfrak{s p i n}(9), \mathfrak{s p}^{*}(n)$ and $\mathfrak{s p}^{*}(n) \oplus \mathfrak{u}(1)$

The transitive action of $\mathfrak{s p i n}(9)$ on $S^{15}$ does not give rise to a KSA for $\mathrm{AdS}_{3}$ backgrounds as there are no $\mathrm{AdS}_{3}$ backgrounds preserving 32 supersymmetries. It remains to investigate the cases $\mathfrak{s p}^{*}(n), \mathfrak{s p}^{*}(n) \oplus \mathfrak{u}(1)$ and $\mathfrak{s p}^{*}(n) \oplus \mathfrak{s p}^{*}(1)$ for which $N=N_{\sigma}=4 n$.

To begin, the embedding of $\mathfrak{s p}^{*}(n)$ in $\mathfrak{s o}(4 n)$ is specified by a hyper-complex structure $I, J$ and $K, I^{2}=J^{2}=-1, I J=-J I$ and $K=I J$ in $\mathbb{R}^{4 n}$. The generators of $\mathfrak{s p}^{*}(n)$ are those of $\mathfrak{s o}(4 n)$ which are $(1,1)$ with respect to all complex structures. Thus we write $\mathfrak{t}_{0}=\hat{\mathfrak{t}}_{0}^{(1,1)} \oplus \mathfrak{m}_{0}$, where $\hat{\mathfrak{t}}_{0}^{(1,1)}=\mathfrak{s p}^{*}(n)$ and $\mathfrak{m}_{0}$ is the orthogonal complement.

The most general 4 -form $\alpha$ which is invariant under $\mathfrak{s p}^{*}(n)$ can be chosen as

$$
\alpha=a_{1} I \wedge I+a_{2} J \wedge J+a_{3} K \wedge K+b_{1} I \wedge J+b_{2} I \wedge K+b_{3} J \wedge K
$$

where we denote with the same symbol the complex structures and their associated Hermitian 2-forms, and $a_{1}, a_{2}, a_{3}, b_{1}, b_{2}, b_{3}$ are constants. Next we impose the condition that the elements of $\mathfrak{m}_{0}$ commute with the $Q$ 's. In particular, we impose the condition that the elements of $\mathfrak{t}_{0}$ which are $(2,0)$ and $(0,2)$ with respect to $I$, and so lie in $\mathfrak{m}_{0}$, must commute with the $Q$ 's. We find

$$
\begin{aligned}
& 2\left(\frac{1}{2}-a_{1}\right)\left(\delta_{p[r} \delta_{s] q}+I_{p[r} I_{s] q}\right)-\frac{1}{2} a_{2}\left((J \wedge J)_{r s p q}-(K \wedge K)_{r s p q}+2 K_{r s} K_{p q}+2 J_{r s} J_{p q}\right) \\
& -\frac{1}{2} a_{3}\left(-(J \wedge J)_{r s p q}+(K \wedge K)_{r s p q}+2 K_{r s} K_{p q}+2 J_{r s} J_{p q}\right) \\
& -\frac{1}{2} b_{1}\left((I \wedge J)_{r s p q}+J_{r s} I_{p q}-I_{r s} J_{p q}+2 \delta_{q[r} K_{s] p}-2 K_{q[r} \delta_{s] p}\right) \\
& -\frac{1}{2} b_{2}\left((I \wedge K)_{r s p q}+K_{r s} I_{p q}-I_{r s} K_{p q}-2 \delta_{q[r} J_{s] p}+2 J_{q[r} \delta_{s] p}\right) \\
& -b_{3}(J \wedge K)_{r s p q}=0 .
\end{aligned}
$$

Taking the trace with $I_{p q}$, one gets that $b_{1}=b_{2}=0$ for $n>1$. Taking the trace with $J_{p q}$ one finds that

$$
\frac{1}{2}-a_{1}-(2 n-1) a_{2}-a_{3}=0, \quad b_{3}=0 .
$$

Next skew-symmetrizing in all $r, s, p$ and $q$ indices and considering the $(4,0)$ and $(2,2)$ parts with respect to $I$, one deduces that after using the above equation that

$$
a_{1}=\frac{1}{2}, \quad a_{2}=a_{3}=0 .
$$

Thus if $n \neq 1$, the conditions above imply $b_{1}=b_{2}=b_{3}=a_{2}=a_{3}=0$ and $a_{1}=\frac{1}{2}$. Thus, the commutator $[\tilde{V}, Q]$ is as in the $\mathfrak{u}(2 n)$ case. However $\mathfrak{s p}^{*}(n) \subset \mathfrak{s u}(2 n)$ and so the trace with respect to $I$ must vanish as well. We have seen that this is not possible. So there is no KSA that can be constructed using the action of $\mathfrak{s p}^{*}(n)$ on the spheres.

The embedding of $\mathfrak{s p}^{*}(n) \oplus \mathfrak{u}(1)$ in $\mathfrak{s o}(4 n)$ is again characterized by the complex structures $I, J$ and $K$ but now $\tilde{V}_{r s} \in \mathfrak{s p}^{*}(n) \oplus \mathfrak{u}(1)$ iff $\tilde{V}_{r s}$ is $(1,1)$ with respect to $I$ and the 
I-traceless part of $\tilde{V}_{r s}, \tilde{V}_{r s}-\frac{1}{4 n} I_{r s} I^{r^{\prime} s^{\prime}} \tilde{V}_{r^{\prime} s^{\prime}}$, is $(1,1)$ with respect to both $J$ and $K$. The most general $\mathfrak{s p}^{*}(n) \oplus \mathfrak{u}(1)$ invariant 4 -form is

$$
\alpha=a_{1} I \wedge I+a_{2}(J \wedge J+K \wedge K),
$$

ie $a_{2}=a_{3}$ and $b_{1}=b_{2}=b_{3}=0$ in $(3.37)$. As $\tilde{V}_{r s}$ is $(1,1)$ with respect to $I$, one can repeat the computation above to show that $a_{2}=a_{3}=0$ and $a_{1}=\frac{1}{2}$. To demonstrate that a superalgebra cannot be constructed from the $\mathfrak{s p}^{*}(n) \oplus \mathfrak{u}(1)$ action on a sphere, take a complex basis with respect to $I$ to find

$$
\left[\tilde{V}_{\beta \bar{\gamma}}, Q_{A \zeta}\right]=-\ell^{-1}\left(-2 \delta_{\zeta \bar{\gamma}} Q_{A \beta}+\delta_{\beta \bar{\gamma}} Q_{A \zeta}\right) .
$$

This allows the computation of the commutator of $\tilde{V}_{r s}-\frac{1}{4 n} I_{r s} I^{r^{\prime} s^{\prime}} \tilde{V}_{r^{\prime} s^{\prime}}$ on the $Q$ 's. Imposing next that the $(2,0)+(0,2)$ component of $\tilde{V}_{r s}-\frac{1}{4 n} I_{r s} I^{r^{\prime} s^{\prime}} \tilde{V}_{r^{\prime} s^{\prime}}$ with respect to $J$ has to commute with the $Q$ 's leads to a contradiction. There are no KSA associated to the $\mathfrak{s p}^{*}(n) \oplus \mathfrak{u}(1)$ case.

\subsection{2 $\mathfrak{s p}^{*}(n) \oplus \mathfrak{s p}^{*}(1)$}

The elements in $\mathfrak{s p}^{*}(n) \oplus \mathfrak{s p}^{*}(1) \subseteq \mathfrak{t}_{0}$ can be written as

$$
\tilde{V}_{r s}=\hat{V}_{r s}+W_{I} I_{r s}+W_{J} J_{r s}+W_{K} K_{r s},
$$

where $\hat{V}_{r s} \in \mathfrak{s p}^{*}(n)$ are $(1,1)$ with respect to all $I, J$ and $K$ while $W_{I}, W_{J}$ and $W_{K}$ are the generators of $\mathfrak{s p}^{*}(1)$. The invariant 4 -form is

$$
\alpha=a(I \wedge I+J \wedge J+K \wedge K),
$$

ie $a_{1}=a_{2}=a_{3}=a$ and $b_{1}=b_{2}=b_{3}=0$ in (3.37).

To proceed, consider the decomposition $\mathfrak{t}_{0}=\mathfrak{s p}^{*}(n) \oplus \mathfrak{s p}^{*}(1) \oplus \mathfrak{m}_{0}$. We have to demonstrate that the elements of $\mathfrak{m}_{0}$ commute with the $Q$ 's, i.e. $\left[\mathfrak{m}_{0}, \mathfrak{g}_{1}\right]=0$. Following (3.30) denote the projections onto the $(2,0)+(0,2)$ and $(1,1)$ subspaces of the space of 2 -forms with respect to the complex structure $I$ with $P_{I}^{(2,0)+(0,2)}$ and $P_{I}^{(1,1)}$, respectively, and similarly for $J$ and $K$. Then note that

$$
P_{I}^{(2,0)+(0,2)} \mathfrak{t}_{0}=\mathfrak{w}_{J} \oplus \mathfrak{w}_{K} \oplus P_{I}^{(2,0)+(0,2)} \mathfrak{m}_{0}
$$

and

$$
P_{J}^{(2,0)+(0,2)} \mathfrak{t}_{0}=\mathfrak{w}_{I} \oplus \mathfrak{w}_{K} \oplus P_{J}^{(2,0)+(0,2)} \mathfrak{m}_{0}
$$

with

$$
\mathfrak{m}_{0}=P_{I}^{(2,0)+(0,2)} \mathfrak{m}_{0}+P_{J}^{(2,0)+(0,2)} \mathfrak{m}_{0},
$$

where $\mathfrak{w}_{I}, \mathfrak{w}_{J}$ and $\mathfrak{w}_{K}$ are the subspaces spanned by the generators $W_{I}, W_{J}$ and $W_{K}$, respectively, ie $\mathfrak{s p}^{*}(1)=\mathfrak{w}_{I} \oplus \mathfrak{w}_{J} \oplus \mathfrak{w}_{K}$. Using (3.45) and (3.46) and after setting $a=1 / 2$, we find that

$$
\begin{aligned}
& {\left[\left(P_{I}^{(2,0)+(0,2)} \tilde{V}\right)_{r s}, Q_{A t}\right]=\ell^{-1}\left(J_{r s} J_{t}^{p} Q_{A p}+K_{r s} K_{t}^{p} Q_{A p}\right)} \\
& {\left[\left(P_{J}^{(2,0)+(0,2)} \tilde{V}\right)_{r s}, Q_{A t}\right]=\ell^{-1}\left(I_{r s} I_{t}^{p} Q_{A p}+K_{r s} K_{t}^{p} Q_{A p}\right) .}
\end{aligned}
$$


Therefore we deduce that

$$
\left[W_{J}, Q_{A t}\right]=\ell^{-1} J_{t}^{p} Q_{A p}, \quad\left[W_{K}, Q_{A t}\right]=\ell^{-1} K_{t}^{p} Q_{A p}, \quad\left[W_{I}, Q_{A t}\right]=\ell^{-1} I_{t}^{p} Q_{A p},
$$

and

$$
\left[P_{I}^{(2,0)+(0,2)} \mathfrak{m}_{0}, \mathfrak{g}_{1}\right]=0, \quad\left[P_{J}^{(2,0)+(0,2)} \mathfrak{m}_{0}, \mathfrak{g}_{1}\right]=0
$$

Then it follows from (3.47) that $\left[\mathfrak{m}_{0}, \mathfrak{g}_{1}\right]=0$.

If the elements of $\mathfrak{m}_{0}$ are set to zero, the resulting superalgebra is isomorphic to the real form, $\mathfrak{o s p}^{*}(4 \mid 2 n)$, of $\mathfrak{o s p}(4 \mid 2 n)$. To see this note that $\mathfrak{o s p}^{*}(4 \mid 2 n)_{0}=\mathfrak{s p}(2) \oplus \mathfrak{s p}^{*}(1) \oplus \mathfrak{s p}^{*}(n)$ while $\mathfrak{o s p}(4 \mid 2 n)_{0}=\mathfrak{s p}(2 n) \oplus \mathfrak{s o}(4)=\mathfrak{s p}(2 n) \oplus \mathfrak{s o}(3) \oplus \mathfrak{s o}(3)$. Then $\mathfrak{s p}^{*}(n)$ and $\mathfrak{s p}(2)$ are real forms of $\mathfrak{s p}(2 n)$ and $\mathfrak{s o}(3)$, respectively, and $\mathfrak{s p}^{*}(1)=\mathfrak{s o}(3)$. On the other hand if the elements of $\mathfrak{m}_{0}$ do not vanish, the KSA may have central terms. It is also known that there are no maximally supersymmetric $\mathrm{AdS}_{3}$ backgrounds and so $n$ is restricted as $n<4$. This completes the identification of all KSA for $\mathrm{AdS}_{3}$ backgrounds. In appendix D, we present some examples for cases with a low number of supersymmetries without using the classification results of [10] that confirm the results we have presented.

We conclude this section by stating all superalgebras of $\mathrm{AdS}_{3}$ backgrounds which preserve 16 supersymmetries. After taking into account the possibility of having both $\sigma$ and $\tau$ Killing spinors can occur, $\mathfrak{g}=\mathfrak{g}_{L} \oplus \mathfrak{g}_{R}$, and setting the central terms to zero, one finds that the KSAs are

$$
\begin{aligned}
& \mathfrak{o} \mathfrak{s p}(8 \mid 2), \quad \mathfrak{s l}(4 \mid 2), \quad \mathfrak{f}(4), \quad \mathfrak{o s p}^{*}(4 \mid 4), \quad \mathfrak{o} \mathfrak{s p}(1 \mid 2) \oplus \mathfrak{g}(3), \quad \mathfrak{o s p}(1 \mid 2) \oplus \mathfrak{o s p}(7 \mid 2), \\
& \mathfrak{o s p}(2 \mid 2) \oplus \mathfrak{o} \mathfrak{s p}(6 \mid 2), \quad \mathfrak{o s p}(2 \mid 2) \oplus \mathfrak{s l}(3 \mid 2), \quad \mathfrak{o} \mathfrak{s p}(3 \mid 2) \oplus \mathfrak{o s p}(5 \mid 2), \\
& \mathfrak{o} \mathfrak{s p}(4 \mid 2) \oplus \mathfrak{o s p}(4 \mid 2) \quad \mathfrak{o} \mathfrak{s p}(4 \mid 2) \oplus \mathfrak{s l}(2 \mid 2) / 1_{4 \times 4}, \quad \mathfrak{s l}(2 \mid 2) / 1_{4 \times 4} \oplus \mathfrak{s l}(2 \mid 2) / 1_{4 \times 4}, \\
& \mathfrak{o s p}(4 \mid 2) \oplus \mathfrak{D}(2,1, \alpha), \quad \mathfrak{s l}(2 \mid 2) / 1_{4 \times 4} \oplus \mathfrak{D}(2,1, \alpha), \mathfrak{D}(2,1, \alpha) \oplus \mathfrak{D}(2,1, \alpha),
\end{aligned}
$$

where we have stated the unordered pairs. Otherwise one has for example to include both $\mathfrak{o s p}(1 \mid 2) \oplus \mathfrak{g}(3)$ and $\mathfrak{g}(3) \oplus \mathfrak{o s p}(1 \mid 2)$ as distinct possibilities. Similar lists can be obtained for any number of supersymmetries.

\section{Heterotic backgrounds}

\subsection{Killing spinors}

Under some mild assumptions, the heterotic string supergravity admits only $\mathrm{AdS}_{3}$ solutions and the warp factor is constant [17]. The solutions preserve 2, 4, 6 and 8 supersymmetries. The Killing spinors of such backgrounds are either expressed in terms of $\sigma_{ \pm}$or $\tau_{ \pm}$spinors as in (3.1) or (3.4), respectively. As only either $\sigma_{ \pm}$or $\tau_{ \pm}$Killing spinors can occur, we shall focus on the Killing spinors expressed in terms of $\sigma_{ \pm}$as the investigation of the KSAs in terms of the $\tau_{ \pm}$Killing spinors is similar. Amongst the conditions (2.9), (2.12) and (2.10) that we have put on the bilinears, the only relevant one is (2.12). This also follows from the gravitino KSE as the connection has holonomy contained in the $\operatorname{Spin}(8)$ group. In 
$\mathrm{AdS}_{3} \mathrm{KSAs}$ in type II and $d=11$

\begin{tabular}{|c|c|c|}
\hline$N_{\sigma} / 2$ & $\mathfrak{g}_{L} / \mathfrak{c}$ & $\mathfrak{t}_{0} / \mathfrak{c}$ \\
\hline$n$ & $\mathfrak{o s p}(n \mid 2)$ & $\mathfrak{s o}(n)$ \\
\hline $2 n, n>1$ & $\mathfrak{s l}(n \mid 2)$ & $\mathfrak{u}(n)$ \\
\hline $4 n, n>1$ & $\mathfrak{o s p}^{*}(4 \mid 2 n)$ & $\mathfrak{s p}^{*}(n) \oplus \mathfrak{s p}(1)$ \\
\hline 8 & $\mathfrak{f}(4)$ & $\mathfrak{s p i n}(7)$ \\
\hline 7 & $\mathfrak{g}(3)$ & $\mathfrak{g}_{2}$ \\
\hline 4 & $\mathfrak{D}(2,1, \alpha)$ & $\mathfrak{s o}(3) \oplus \mathfrak{s o}(3)$ \\
\hline 4 & $\mathfrak{s l}(2 \mid 2) / 1_{4 \times 4}$ & $\mathfrak{s o}(3)$ \\
\hline
\end{tabular}

Table 2. In all cases, $\left(\mathfrak{g}_{L} / \mathfrak{c}\right)_{0}=\mathfrak{s o}(1,2) \oplus \mathfrak{t}_{0} / \mathfrak{c}$. It is required that $N_{\sigma} / 2<16$ as there are no maximally supersymmetric $\mathrm{AdS}_{3}$ backgrounds.

particular one does not have to assume the compactness of the internal space or use the maximum principle.

The KSAs of the heterotic $\mathrm{AdS}_{3}$ backgrounds can be easily constructed from first principles without using the super-Jacobi identities we have employed in the type II theories. This is mainly due to the observation that the solution of the gravitino KSE puts strong restrictions on Killing spinors namely that they should have a non-trivial isotropy group in $\operatorname{Spin}(9,1)$. The anti-commutator of odd generators can be read from the results of appendix C. Moreover, the commutator of even and odd generators can be easily found. Indeed upon using the gravitino KSE the spinorial Lie derivative of any Killing spinor $\epsilon$ with respect to any 1-form bilinear $X$ can be expressed as

$$
\mathcal{L}_{X} \epsilon=\frac{1}{4} i_{X} \text { H } \epsilon,
$$

where $H$ is the 3 -form field strength. As both $\epsilon$ and $H$ are known in all cases, the righthand-side of this equation can be easily evaluated.

\subsection{KSAs for heterotic backgrounds}

The KSAs of $N=2,4$ and $N=6 \mathrm{AdS}_{3}$ backgrounds can be either constructed from first principles as described in the previous section or can be read from the results we have already presented for the type II backgrounds. In either case, they are unique and isomorphic to $\mathfrak{o} \mathfrak{s p}(1 \mid 2), \mathfrak{o} \mathfrak{s p}(2 \mid 2)$ and $\mathfrak{o s p}(3 \mid 2)$, respectively.

It remains to investigate the $N=8 \mathrm{AdS}_{3}$ backgrounds. As in type II theories, the Killing spinors are given in (3.6) but now $r=1,2,3,4$. Furthermore, we express $K_{A r, B s}$ as in (3.7) and there are potentially six 1 -forms $\tilde{K}_{r s}=-\tilde{K}_{s r}$ along the internal space. The associated vector fields of these commute with those of $K_{A B}$. However, the Killing spinors of $N=8$ heterotic $\mathrm{AdS}_{3}$ backgrounds are restricted to be $\mathfrak{s u}(2)$ invariant and such backgrounds admit only three 1 -form bilinears along the internal space [17]. As a consequence only three of the six 1 -forms $\tilde{K}_{r s}$ are linearly independent. This is imposed by requiring that $\tilde{K}_{r s}$ is self-dual, ie

$$
\tilde{K}_{r s}=\frac{1}{2} \epsilon_{r s}^{p q} \tilde{K}_{p q},
$$




\begin{tabular}{|c|c|c|}
\hline$N$ & $\mathfrak{g}$ & $\mathfrak{t}_{0}$ \\
\hline 2 & $\mathfrak{o} \mathfrak{s p}(1 \mid 2)$ & - \\
\hline 4 & $\mathfrak{o} \mathfrak{s p}(2 \mid 2)$ & $\mathfrak{s o}(2)$ \\
\hline 6 & $\mathfrak{o s p}(3 \mid 2)$ & $\mathfrak{s o}(3)$ \\
\hline 8 & $\mathfrak{s l}(2 \mid 2) / 1_{4 \times 4}$ & $\mathfrak{s o}(3)$ \\
\hline
\end{tabular}

Table 3. Heterotic $\mathrm{AdS}_{3} \mathrm{KSAs}$.

for some choice of ordering of Killing spinors. The commutator $\left[\tilde{V}_{r s}, Q_{A t}\right]$ can either be found from explicitly computing the spinorial Lie derivative of $\tilde{K}_{r s}$ using (4.1) and the form of the 3-form flux for such backgrounds given in [17] or it can be read from the results for type II backgrounds as this case corresponds to the $\hat{\alpha}=-1$ case in (3.27). In either case writing $\tilde{V}_{r s}=\left(\omega_{P}^{(+)}\right)_{r s} \tilde{V}_{P}, P=1,2,3$, where $\omega_{P}^{(+)}$is a orthonormal basis in the space of self-dual 2-forms in $\mathbb{R}^{4}$ such that $\left[\omega_{P}^{(+)}, \omega_{Q}^{(+)}\right]=-2 \epsilon_{P Q} \omega_{S}^{(+)}$, one finds that

$$
\begin{aligned}
{\left[\tilde{V}_{P}, Q_{A r}\right] } & =-\ell^{-1}\left(\omega_{P}^{(+)}\right)_{r}{ }^{s} Q_{A s} \\
{\left[\tilde{V}_{P}, \tilde{V}_{Q}\right] } & =-\ell^{-1} \epsilon_{P Q}{ }^{S} \tilde{V}_{S}
\end{aligned}
$$

This is a real form of the $\mathfrak{s l}(2 \mid 2) / 1_{4 \times 4}$ superalgebra and the isometry algebra of the internal space is $\mathfrak{s o}(3)$. Central charges do not arise in the heterotic case. The KSAs of $\mathrm{AdS}_{3}$ heterotic backgrounds are tabulated in table 3 .

\section{$5 \quad \mathrm{AdS}_{4}$ in $\mathrm{D}=11$ and type II theories}

\section{$5.1 \quad N=4$}

$\mathrm{AdS}_{4}$ backgrounds preserve $4 k$ supersymmetries so $N=4$ is the minimal case. Choosing the coordinates of $\mathrm{AdS}_{4}$ as $(u, r, z, x)$, the four Killing spinors (2.4) and (2.5) can be written as

$$
\begin{aligned}
& \epsilon_{1}=\sigma_{+}, \quad \epsilon_{2}=A \Gamma_{-z} \sigma_{+}+2 \ell^{-1} u \sigma_{+}-\ell^{-1} x A e^{z / \ell} \Gamma_{-x} \sigma_{+} \\
& \epsilon_{3}=e^{-z / \ell}\left(\Gamma_{z x} \sigma_{+}-\ell^{-1} r A^{-1} \Gamma_{-x} \sigma_{+}\right)-\ell^{-1} x \sigma_{+}, \quad \epsilon_{4}=A e^{z / \ell} \Gamma_{-x} \sigma_{+},
\end{aligned}
$$

where we have used $\sigma_{-}=A \Gamma_{-z} \sigma_{+}, \tau_{+}=\Gamma_{z x} \sigma_{+}$and $\tau_{-}=A \Gamma_{-x} \sigma_{+}$.

The 1-form bilinears have been computed in appendix C. They span all ten isometries of $\mathrm{AdS}_{4}$. Furthermore, all 1-form bilinears associated with isometries of the internal space vanish. The spinorial Lie derivatives of the Killing spinors along the isometries of $\mathrm{AdS}_{4}$ can be easily extracted from the formulae in appendix C. It turns out that the resulting $\mathrm{KSA}$ is

$$
\left\{Q_{A}, Q_{B}\right\}=V_{A B}, \quad\left[V_{A B}, Q_{C}\right]=-\ell^{-1}\left(\epsilon_{C A} Q_{B}+\epsilon_{C B} Q_{A}\right)
$$

where $A, B, C=1, \ldots, 4, \epsilon_{A B}$ is the symplectic invariant 2-form put into canonical form with $\epsilon_{12}=-\epsilon_{34}=1$ and the spinor $\sigma_{+}$has been normalized as $2\left\|\sigma_{+}\right\|^{2}=1$. This superalgebra is isomorphic to $\mathfrak{o s p}(1 \mid 4)$. 


\section{$5.2 \quad N=8$}

The Killing spinors of $N=8 \mathrm{AdS}_{4}$ backgrounds are generated by the choice of two linearly independent spinors $\sigma_{+}^{r}, r=1,2$. As each $\sigma_{+}$generates four Killing spinors as in (5.1), we shall denote the Killing spinors with $\epsilon_{A r}$. Without loss of generality $\sigma_{+}^{r}$ can be chosen to be orthogonal $2\left\langle\sigma_{+}^{r}, \sigma_{+}^{s}\right\rangle=\delta_{r s}$. Furthermore a direct application of the formulae in appendix $\mathrm{C}$ reveals that

$$
\left\{Q_{A r}, Q_{B s}\right\}=\delta_{r s} V_{A B}+\epsilon_{A B} \tilde{V}_{r s},
$$

where $\tilde{V}_{r s}=-\tilde{V}_{s r}$. The generators $V_{A B}$ are associated to isometries along $\mathrm{AdS}_{4}$ as in the minimal $N=4$ case and $\tilde{V}_{r s}$ is a generator associated to the 1-form bilinear

$$
\tilde{K}_{r s}=2 A\left\langle\sigma_{+}^{r}, \Gamma_{z i} \sigma_{+}^{s}\right\rangle \mathbf{e}^{i},
$$

which gives rise to a Killing vector field along the internal space. Note that $\tilde{V}_{r s}$ commutes with $V_{A B}$.

A further direct computation using the formulae in appendix $\mathrm{C}$ also reveals that

$$
\left[V_{A B}, Q_{C r}\right]=-\ell^{-1}\left(\epsilon_{C A} Q_{B r}+\epsilon_{C B} Q_{A r}\right) .
$$

It remains to determine the commutators $[\tilde{V}, Q]$. For this, we shall use the closure of the KSA as an explicit computation will require further details of the geometry of the internal space that are not available. As $\tilde{K}$ is along the internal directions it preserves the functional dependence of Killing spinors $\epsilon_{A r}$ on $\mathrm{AdS}_{4}$ coordinates. So $\mathcal{L}_{\tilde{K}_{r s}} \epsilon_{A t}=\beta_{r s t} \epsilon_{A \ell}$ for some constants $\beta$. Then one can compute $[\tilde{V}, Q]$ using the super-Jacobi identities. In particular an argument similar to that employed for the $\operatorname{AdS}_{3} N>2$ backgrounds to prove that $\alpha$ is a 4-form, together with (5.3) and (5.5) leads to

$$
\left[\tilde{V}_{r s}, Q_{A t}\right]=-\ell^{-1}\left(\delta_{t r} Q_{A s}-\delta_{t s} Q_{A r}\right) .
$$

The KSA given by the (anti)-commutators (5.3), (5.5) and (5.6) is isomorphic to $\mathfrak{o s p}(2 \mid 4)$.

\section{$5.3 \quad N=12$}

The Killing spinors $\epsilon_{A r}$ of these backgrounds are given as in (5.1) and depend on three spinors $\sigma_{+}^{r}, r=1,2,3$, which without loss of generality we can choose as $2\left\langle\sigma_{+}^{r}, \sigma_{+}^{s}\right\rangle=\delta_{r s}$.

Using the results in appendix $\mathrm{C}$, one can show that the anticommutators of the $Q_{A r}$ generators are

$$
\left\{Q_{A r}, Q_{B s}\right\}=\delta_{r s} V_{A B}+\epsilon_{A B} \tilde{V}_{r s},
$$

where now the three generators $\tilde{V}_{r s}, \tilde{V}_{r s}=-\tilde{V}_{s r}$, are associated with the three 1-form bilinears

$$
\tilde{K}_{r s}=2 A\left\langle\sigma_{+}^{r}, \Gamma_{z i} \sigma_{+}^{s}\right\rangle \mathbf{e}^{i},
$$

which in turn give rise to Killing vector fields in the internal space. Similarly the commutator of $V$ and $Q$ generators is given as in (5.5). It remains to determine the commutators 
$\left[\tilde{V}_{r s}, Q_{A t}\right]$. Again we shall use the closure of KSA for this. If $t$ is equal to either $r$ or $s$, then a similar argument to that used for $N=8$ gives that the commutator is as in (5.6). It remains to determine the commutators for $t \neq r, s$. As $\tilde{K}_{r s}$ are along the internal space, the spinorial Lie derivative preserves the functional dependence of the Killing spinors on the $\mathrm{AdS}_{4}$ coordinates and so the commutator $\left[\tilde{V}_{r s}, Q_{A t}\right]$ must close to a linear combination of $Q_{A \ell}$ generators. Because of this, it suffices to choose $A=1$ and consider first the case $r=1, s=2$ and $t=3$. Then applying the super-Jacobi identity using (5.7), one finds

$$
\left[\tilde{V}_{12}, Q_{13}\right]=-\left[\left\{Q_{31}, Q_{42}\right\}, Q_{13}\right]=\left[\left\{Q_{13}, Q_{31}\right\}, Q_{42}\right]+\left[\left\{Q_{42}, Q_{13}\right\}, Q_{31}\right]=0 .
$$

Thus $\left[\tilde{V}_{12}, Q_{A 3}\right]=0$ as well. A similar argument implies that $\left[\tilde{V}_{r s}, Q_{A t}\right]=0$ for $t \neq r, s$. As a result the commutator is

$$
\left[\tilde{V}_{r s}, Q_{A t}\right]=-\ell^{-1}\left(\delta_{t r} Q_{A s}-\delta_{t s} Q_{A r}\right) .
$$

The argument presented above to determine the commutator (5.10) is general and does not depend of the range of the indices $r, s, t$. Clearly, the KSAs of $\mathrm{AdS}_{4}$ backgrounds with extended supersymmetry are more restricted than those $\mathrm{AdS}_{3}$ backgrounds. The key reason for this is that unlike the $\mathrm{AdS}_{3}$ case, the generators $\tilde{V}_{r s}$ can be written as anti-commutators of odd generators in two different ways for $A=1, B=2$ and for $A=3, B=4$.

The commutator $\left[\tilde{V}_{r s}, \tilde{V}_{r^{\prime} s^{\prime}}\right]$ can be easily computed using the super-Jacobi identities to reveal that

$$
\left[\tilde{V}_{r s}, \tilde{V}_{r^{\prime} s^{\prime}}\right]=-\ell^{-1}\left(\delta_{r r^{\prime}} \tilde{V}_{s s^{\prime}}-\delta_{s r^{\prime}} \tilde{V}_{r s^{\prime}}-\delta_{r s^{\prime}} \tilde{V}_{s r^{\prime}}+\delta_{s s^{\prime}} \tilde{V}_{r r^{\prime}}\right)
$$

ie the Lie algebra of the Killing vector fields of the internal space is $\mathfrak{s o}(3)$. The KSA of $\mathrm{AdS}_{4}$ backgrounds with $N=12$ supersymmetries is isomorphic to $\mathfrak{o} \mathfrak{s p}(3 \mid 4)$.

\section{$\mathbf{5 . 4} \quad N=16$}

The Killing spinors $\epsilon_{A r}$ are again given as in (5.1) but now determined by similarly normalized spinors $\sigma_{+}^{r}$ for $r=1,2,3,4$. The squaring operation of the Killing spinors which gives the anti-commutators $\left\{Q_{A r}, Q_{B s}\right\}$ leads to an expression as in (5.7) but now for $r, s=1,2,3,4$. The generators $\tilde{V}_{r s}$ are associated with Killing vectors along the internal space. Also the commutator $\left[V_{A B}, Q_{C r}\right]$ is given as in (5.5).

The remaining commutators which need to be determined are $\left[\tilde{V}_{r s}, Q_{A t}\right]$. If $t=r$ or $t=s$, an argument similar to the one produced for the $N=8$ case leads to a commutator as in (5.6). On the other hand if all $r, s, t$ are distinct, a similar argument to that used in the $N=12$ case implies that the commutator vanishes. Thus the commutator is given as in (5.10) but now for $r, s, t=1,2,3,4$. Furthermore as a consequence of this and the superJacobi identities the Lie algebra of $V_{r s}$ is as in (5.11), i.e. isomorphic to $\mathfrak{s o}(4)$. Therefore the KSA of $N=16$ backgrounds is isomorphic to $\mathfrak{o s p}(4 \mid 4)$.

\section{5 $\quad N>16$}

It is straightforward to generalize the results we have obtained so far to all $N>16$ backgrounds. One can show that the KSA of $N=4 k$ backgrounds is $\mathfrak{o s p}(k \mid 4)$. The non- 
vanishing commutators are

$$
\begin{aligned}
\left\{Q_{A r}, Q_{B s}\right\} & =\delta_{r s} V_{A B}+\epsilon_{A B} \tilde{V}_{r s} \\
{\left[V_{A B}, Q_{C r}\right] } & =-\ell^{-1}\left(\epsilon_{C A} Q_{B r}+\epsilon_{C B} Q_{A r}\right) \\
{\left[\tilde{V}_{r s}, Q_{A t}\right] } & =-\ell^{-1}\left(\delta_{t r} Q_{A s}-\delta_{t s} Q_{A r}\right) \\
{\left[\tilde{V}_{r s}, \tilde{V}_{r^{\prime} s^{\prime}}\right] } & =-\ell^{-1}\left(\delta_{r r^{\prime}} \tilde{V}_{s s^{\prime}}-\delta_{s r^{\prime}} \tilde{V}_{r s^{\prime}}-\delta_{r s^{\prime}} \tilde{V}_{s r^{\prime}}+\delta_{s s^{\prime}} \tilde{V}_{r r^{\prime}}\right)
\end{aligned}
$$

where $A, B, C=1, \ldots, 4$ and $r, s, t, r^{\prime}, s^{\prime}=1, \ldots, k$. The algebra of the Killing vector fields of the internal space is $\mathfrak{s o}(k)$.

We remark that the KSAs of $\mathrm{AdS}_{4}$ backgrounds are more restricted than those of $\mathrm{AdS}_{3}$ backgrounds. The critical argument that explains the reason for this is produced below (5.10) and uses in an essential way the fact that the generators of the isometries in the internal space $\tilde{V}_{r s}$ appear as bilinears of both type $\sigma$ and type $\tau$ Killing spinors. Equivalently, the enhanced symmetry of the spacetime imposes more stringent conditions on the remaining (anti-) commutators which are sufficient, together with the super-Jacobi identities, to specify the KSAs including the commutators involving the generators of the isometries of the internal space. This is the case for all KSAs, $\mathfrak{g}$, of $\operatorname{AdS}_{n} n>3$ backgrounds provided that $\mathfrak{g}_{0}=\mathfrak{s o}(n-1,2) \oplus \mathfrak{t}_{0}$.

\section{$6 \quad \mathrm{AdS}_{5}$ in $\mathrm{D}=11$ and type II theories}

It has been shown in [12-14] that warped $\mathrm{AdS}_{5}$ backgrounds preserve $8 k$ supersymmetries. Unlike the AdS solutions we have investigated so far, the minimal $N=8 \mathrm{AdS}_{5}$ backgrounds exhibit a non-trivial isometry along the internal space. Because of this the analysis is somewhat different.

\section{1 $N=8$}

To begin let us take $(r, u, z, x, y)$ as the coordinates of $\mathrm{AdS}_{5}$, where we have set $x^{1}=x$ and $x^{2}=y$. The eight Killing spinors of the minimal $\mathrm{AdS}_{5}$ solution are generated by a single spinor $\sigma_{+}$after applying the elementary operations described in section 2.2. It follows that if $\sigma_{+}$is a Killing spinor, then $\Gamma_{x y} \sigma_{+}$is also a Killing spinor. As a result the Killing spinors can be organized in two pairs of four spinors as $\epsilon_{A I}$ where $A=1, \ldots, 4$ and $I=1,2$. The four $\epsilon_{A 1}$ spinors are as those of the minimal $\mathrm{AdS}_{4}$ backgrounds generated by $\sigma_{+}^{1}=\sigma_{+}$ and $\tau_{+}^{1}=\Gamma_{z x} \sigma_{+}^{1}, \sigma_{-}^{1}=A \Gamma_{-z} \sigma_{+}^{1}, \tau_{-}^{1}=A \Gamma_{-z} \tau_{+}^{1}$. The remaining four Killing spinors $\epsilon_{A 2}$ are generated by $\sigma_{+}^{2}=\Gamma_{x y} \sigma_{+}$after applying the same elementary operations. With these identifications, each of the $\epsilon_{A 1}$ and $\epsilon_{A 2}$ spinors generate a osp $(1 \mid 4)$ superalgebra as in the minimal $\mathrm{AdS}_{4}$ case. Then a direct substitution into the vector bilinears of appendix $\mathrm{C}$ reveals that

$$
\left\{Q_{A I}, Q_{B J}\right\}=\delta_{I J} V_{A B}+\epsilon_{I J} \stackrel{\circ}{W}_{A B}+\epsilon_{I J} \epsilon_{A B} K
$$

where $\stackrel{\circ}{W}_{A B} \epsilon^{A B}=0, \epsilon_{A B}$ is the $\mathfrak{s p}(4)$ invariant tensor defined as in the $\mathrm{AdS}_{4}$ case with $\epsilon_{A B} \epsilon^{A C}=\delta_{B}^{C}$, and $\epsilon_{I J}=-\epsilon_{J I}$. There are 16 linearly independent 1-form bilinears. Ten are associated to $V_{A B}$ and five are associated to $\stackrel{\circ}{W}_{A B}$. These span the fifteen isometries 
of $\mathrm{AdS}_{5} . K$ on the other hand is associated with an isometry along the internal space generated by 1 -form bilinear $K=2 A\left\langle\sigma_{+}, \Gamma_{x y z i} \sigma_{+}\right\rangle \mathbf{e}^{i}$. The Lie algebra of the isometries is $\mathfrak{s o}(2,4) \oplus \mathfrak{s o}(2)$.

It remains to compute the commutators of $V, \stackrel{\circ}{W}$ and $K$ with the $Q$ 's. The commutators of $V$ and ${ }^{\circ}$ with the $Q$ 's can be read from the spinorial derivatives on the Killing spinors presented in appendix $\mathrm{C}$ as these even generators are associated with isometries of $\mathrm{AdS}_{5}$. In particular it is straightforward to see that

$$
\left[V_{A B}, Q_{C I}\right]=-\ell^{-1}\left(\epsilon_{C A} Q_{B I}+\epsilon_{C B} Q_{A I}\right) .
$$

It remains to find the commutator of $K$ with the $Q$ 's. For this observe that

$$
\left\{Q_{11}, Q_{22}\right\}=-\left\{Q_{12}, Q_{21}\right\}=M+K, \quad\left\{Q_{31}, Q_{42}\right\}=-\left\{Q_{32}, Q_{41}\right\}=M-K,
$$

where the generator $M$ is associated with the 1-form bilinear $-\ell^{-1} M^{x y}$. We have used the normalization $2\left\|\sigma_{+}\right\|^{2}=1$. Thus for every $Q_{A I}$ there is another odd generator $Q_{A^{\prime} I^{\prime}}$ such that $\left\{Q_{A I}, Q_{A^{\prime} I^{\prime}}\right\} \propto M \pm K$. This gives

$$
\left[M \pm K, Q_{A I}\right] \propto\left[\left\{Q_{A I}, Q_{A^{\prime} I^{\prime}}\right\}, Q_{A I}\right]=-\frac{1}{2}\left[\left\{Q_{A I}, Q_{A I}\right\}, Q_{A^{\prime} I^{\prime}}\right]=\ell^{-1} \epsilon_{A^{\prime} A} Q_{A I^{\prime}} .
$$

As the commutators $\left[M, Q_{A I}\right]$ are known, one can find all the commutators $\left[K, Q_{A I}\right]$. These results can be collected as

$$
\left[W_{A B}, Q_{C I}\right]=-\ell^{-1} \epsilon_{I J}\left(\epsilon_{C A} Q_{B J}-\epsilon_{C B} Q_{A J}+2 \epsilon_{A B} Q_{C J}\right),
$$

where $W_{A B}=\stackrel{\circ}{W}_{A B}+\epsilon_{A B} K$. In particular, one has

$$
\left[K, Q_{A I}\right]=-\frac{3}{2} \ell^{-1} \epsilon_{I J} Q_{A J}
$$

Clearly the generator $K$ cannot be set to zero establishing that the internal spaces of all such backgrounds must have a non-trivial isometry. The (anti)-commutators (6.1), (6.2) and (6.5) determine the KSA and it is isomorphic to $\mathfrak{s l}(1 \mid 4)$.

\section{2 $N=16$}

As the $\mathrm{AdS}_{5}$ backgrounds preserve $8 k$ supersymmetries, the next case to investigate is $N=16$. For this set $\sigma_{+}^{1}=\sigma_{+}$, where $\sigma_{+}$is the Killing spinor of the $N=8$ case and introduce another Killing spinor $\sigma_{+}^{2}$ which is linearly independent from both $\sigma_{+}^{1}$ and $\Gamma_{x y} \sigma_{+}^{1}$. Without loss of generality one can choose $\sigma_{+}^{2}$ to be orthogonal to both $\sigma_{+}^{1}$ and $\Gamma_{x y} \sigma_{+}^{1}$. As a result $\sigma_{+}^{1}, \Gamma_{x y} \sigma_{+}^{1}, \sigma_{+}^{2}$ and $\Gamma_{x y} \sigma_{+}^{2}$ can be chosen as mutually orthogonal.

A direct inspection of the bilinears in appendix $\mathrm{C}$ reveals that the $Q$ anti-commutators can be arranged as

$$
\left\{Q_{A I}^{r}, Q_{B J}^{s}\right\}=\delta_{I J} \delta^{r s} V_{A B}+\epsilon_{I J} \delta^{r s} \stackrel{\circ}{W}_{A B}+\delta_{I J} \epsilon_{A B} \tilde{V}^{r s}+\epsilon_{I J} \epsilon_{A B} K^{r s}
$$

where the $V$ and $\stackrel{\circ}{W}$ generators are as in the $N=8$ case while the generators $\tilde{V}^{r s}=-\tilde{V}^{s r}$ and $K^{r s}=K^{s r}$ are associated to the 1-form bilinears

$$
K^{r s}=2 A\left\langle\sigma_{+}^{r}, \Gamma_{x y z i} \sigma_{+}^{s}\right\rangle \mathbf{e}^{i}, \quad \tilde{V}^{r s}=2 A \operatorname{Re}\left\langle\sigma_{+}^{r}, \Gamma_{z i} \sigma_{+}^{s}\right\rangle \mathbf{e}^{i},
$$

and $r, s=1,2$. 
It remains to investigate the commutators of even and odd generators. All such commutators that involve generators of isometries along the $\mathrm{AdS}_{5}$ can be easily computed using the spinorial Lie derivatives. In particular, one has

$$
\left[\stackrel{\circ}{W}_{A B}, Q_{C I}^{r}\right]=-\ell^{-1} \epsilon_{I J}\left(\epsilon_{C A} Q_{B J}^{r}-\epsilon_{C B} Q_{A J}^{r}+\frac{1}{2} \epsilon_{A B} Q_{C J}^{r}\right) .
$$

Next turn to compute the commutators of generators of isometries along the internal directions with the odd generators. To find the commutator $\left[\tilde{V}^{r s}, Q_{A I}^{t}\right]$, observe that for $I=J$ the anti-commutator (6.7) reduces to that of $N=8 \mathrm{AdS}_{4}$ backgrounds. Then a similar argument to that produced in the $\mathrm{AdS}_{4}$ case leads to the commutator

$$
\left[\tilde{V}^{r s}, Q_{A I}^{t}\right]=-\ell^{-1}\left(\delta^{t r} Q_{A I}^{s}-\delta^{t s} Q_{A I}^{r}\right) .
$$

To find the commutators of $\left[K^{r s}, Q_{A I}^{t}\right]$, observe that

$$
\begin{aligned}
& K^{r s}+\stackrel{\circ}{W}_{12} \delta^{r s}=\left\{Q_{11}^{r}, Q_{22}^{s}\right\}=-\left\{Q_{12}^{r}, Q_{21}^{s}\right\}, \\
& K^{r s}-\stackrel{\circ}{W}_{34} \delta^{r s}=-\left\{Q_{31}^{r}, Q_{42}^{s}\right\}=\left\{Q_{32}^{r}, Q_{41}^{s}\right\}
\end{aligned}
$$

Therefore for each $Q_{A I}^{t}$, there are $Q_{A^{\prime} 1}^{r}$ and $Q_{B^{\prime} 2}^{s}$ such that $\epsilon_{A A^{\prime}}=\epsilon_{A B^{\prime}}=0$ and $K^{r s}$ appears in the anti-commutator $\left\{Q_{A^{\prime} 1}^{r}, Q_{B^{\prime} 2}^{s}\right\}$. Using this we have

$$
\begin{aligned}
{\left[K^{r s} \epsilon_{A^{\prime} B^{\prime}}+\delta^{r s} \stackrel{\circ}{W}_{A^{\prime} B^{\prime}}, Q_{A I}^{t}\right] \propto } & {\left[\left\{Q_{A^{\prime} 1}^{r}, Q_{B^{\prime 2}}^{s}\right\}, Q_{A I}^{t}\right]=-\left[\left\{Q_{A I}^{t}, Q_{A^{\prime} 1}^{r}\right\}, Q_{B^{\prime} 2}^{s}\right] } \\
& -\left[\left\{Q_{B^{\prime} 2}^{s}, Q_{A I}^{t}\right\}, Q_{A^{\prime} 1}^{r}\right] .
\end{aligned}
$$

Observe that the right-hand-side of the equation above does not depend on generators $K^{r s}$ and all the (anti-)commutators are known. Thus one can use the above formula to find

$$
\left[K^{r s}, Q_{A I}^{t}\right]=-\ell^{-1} \epsilon_{I J}\left(\delta^{t r} Q_{A J}^{s}+\delta^{t s} Q_{A J}^{r}-\frac{1}{2} \delta^{r s} Q_{A J}^{t}\right) .
$$

This superalgebra defined by the (anti-)commutators (6.7), (6.9), (6.10) and (6.13) is isomorphic to $\mathfrak{s l}(2 \mid 4)$ and the isometry algebra of the transverse space is $\mathfrak{u}(2)$.

\subsection{Extended supersymmetry}

The two remaining cases to investigate are for $N=24$ and for $N=32$. The bilinears that lie along the internal directions are again given as in (6.8) but now for $r, s=1,2,3$ and $r, s=1, \ldots, 4$, respectively. The anti-commutator of the $Q$ generators is again given in (6.7) but now either $r, s=1,2,3$ or $r, s=1, \ldots, 4$, where again the generators $\tilde{V}^{r s}$ and $K^{r s}$ are associated to bilinears that lie along the internal space. The commutators $\left[\tilde{V}^{r s}, Q_{A I}^{t}\right]$ and $\left[K^{r s}, Q_{A I}^{t}\right]$ are again given by (6.10) and (6.13), respectively, for either $r, s=1,2,3$ or $r, s=1, \ldots, 4$. This is because the argument to establish (6.10) and (6.13) for the $N=16$ case is not sensitive to the range of the indices $r, s$ and $t$. The resulting superalgebra in the $N=24$ case is isomorphic to $\mathfrak{s l}(3 \mid 4)$ and the Lie algebra of isometries of the internal space is $\mathfrak{u}(3)$. However, although this local analysis allows for the existence of $N=24$ solutions, a global analysis given in [21] which makes use of a maximum principle argument on the (compact and without boundary) internal space, excludes such solutions. 
In the $N=32$ case observe that the generator $C=\frac{1}{4} \delta_{r s} K^{r s}$ is central as it commutes with all the $Q$ 's and all the even generators. So either $C$ does not appear in the righthand-side of the anti-commutator (6.7) in which case the superalgebra is isomorphic to $\mathfrak{s l}(4 \mid 4) / \lambda 1_{8 \times 8}$ and the isometry algebra of the internal space is $\mathfrak{s u}(4)=\mathfrak{s o}(6)$ or the KSA is not simple. The latter case does not occur as the only maximally supersymmetric $\mathrm{AdS}_{5}$ background is the IIB $\mathrm{AdS}_{5} \times S^{5}$ solution and the effective isometry algebra of the transverse space is $\mathfrak{s o}(6)$. However this cannot be deduced on symmetry considerations alone as the classification of maximal supersymmetric solutions of IIB theory is also required [18].

\section{$7 \quad \mathrm{AdS}_{6}$ in $\mathrm{D}=11$ and type II theories}

$\mathrm{AdS}_{6}$ backgrounds preserve either 16 or 32 supersymmetries. It has been known for some time that there are no $\mathrm{AdS}_{6}$ backgrounds with 32 supersymmetries in 11-dimensional and type II supergravities [18]. As a result the only case that remains to be investigated is that of 16 supersymmetries. For this, let us denote the coordinates of $\operatorname{AdS}_{6}$ with $\left(u, r, z, x^{a}\right)$, $a=1,2,3$, and introduce the odd generators

$$
Q_{A}=Q_{\epsilon_{A}\left(\sigma_{+}\right)}, \quad Q_{A a}=Q_{\frac{1}{2} \epsilon_{a}^{b c} \epsilon_{A}\left(\Gamma_{b c} \sigma_{+}\right)}, \quad \tilde{Q}_{A}=Q_{\epsilon_{A+2}\left(\Gamma_{z 123} \sigma_{+}\right)}, \quad \tilde{Q}_{A a}=Q_{\epsilon_{A+2}\left(\Gamma_{z a} \sigma_{+}\right)}
$$

with $A=1,2$, where we have used the expression of Killing spinors in (2.4) together with $\sigma_{-}=A \Gamma_{-z} \sigma_{+}$and $\tau_{-}=A \Gamma_{-z} \tau_{+}$as well as the relation between $\tau_{+}$and $\sigma_{+}$spinors. The $a, b, c$ indices are raised with respect to the flat metric and $\epsilon_{a b c}$ is the Levi-Civita tensor.

A direct substitution of the Killing spinors into the 1-form bilinears of appendix C reveals that the non-vanishing anti-commutators are

$$
\begin{array}{rll}
\left\{Q_{A}, Q_{B}\right\} & =V_{A B}, \quad\left\{Q_{A}, Q_{B a}\right\}=\frac{1}{2} \epsilon_{A B} \epsilon_{a}^{b c} K_{b c}^{(-)}, & \left\{Q_{A a}, Q_{B b}\right\}=\delta_{a b} V_{A B}+\epsilon_{A B} K_{a b}^{(+)} \\
\left\{\tilde{Q}_{A}, \tilde{Q}_{B}\right\} & =\tilde{V}_{A B}, \quad\left\{\tilde{Q}_{A}, \tilde{Q}_{B a}\right\}=\frac{1}{2} \epsilon_{A B} \epsilon_{a}^{b c} K_{b c}^{(-)}, & \left\{\tilde{Q}_{A a}, \tilde{Q}_{B b}\right\}=\delta_{a b} \tilde{V}_{A B}-\epsilon_{A B} K_{a b}^{(+)} \\
\left\{Q_{A}, \tilde{Q}_{B a}\right\} & =\left\{\tilde{Q}_{B}, Q_{A a}\right\}=V_{A B, a}, & \left\{Q_{A a}, \tilde{Q}_{B b}\right\}=-\epsilon_{a b}{ }^{c} V_{A B, c}
\end{array}
$$

The generators $V_{A B}$ and $\tilde{V}_{A B}$ are associated to 1-form bilinears (3.2) and (3.5), respectively. The generators $K_{a b}^{( \pm)}$are associated to 1-form bilinears

$$
\pm \ell^{-1} M_{a b}+2 A\left\langle\sigma_{+}, \Gamma_{z i} \Gamma_{a b} \sigma_{+}\right\rangle \mathbf{e}^{i}
$$

and the generators $V_{A B, a}$ are associated to the bilinears

$$
V_{11, a}=-\ell^{-1} M^{-}{ }_{a}, \quad V_{12, a}=-\lambda^{a}, \quad V_{21, a}=2 \ell^{-1} M_{a}^{z}+\lambda_{a}, \quad V_{22, a}=2 \ell^{-1} M^{+}{ }_{a}
$$

where $2\left\|\sigma_{+}\right\|^{2}=1$. There are at most 3 Killing vectors along the internal space associated with the bilinears

$$
K_{a b}=2 A\left\langle\sigma_{+}, \Gamma_{z i} \Gamma_{a b} \sigma_{+}\right\rangle \mathbf{e}^{i}, \quad a, b=1,2,3 .
$$

Note that the bilinear $K_{a b c}=2 A\left\langle\sigma_{+}, \Gamma_{a b c} \Gamma_{i} \sigma_{+}\right\rangle \mathbf{e}^{i}$ vanishes as a consequence of the conditions (2.9) in section 2.3. 
As in previous cases, to determine commutators of the KSA it suffices to find the commutators of the generators $K_{a b}$ of the isometries of the internal space with $Q$ 's as the rest follow from the explicit formulae in appendix $\mathrm{C}$ via the evaluation of spinorial Lie derivatives of the Killing spinors along the isometries of $\mathrm{AdS}_{6}$. To find $\left[K_{b c}, Q_{A a}\right]$ and $\left[K_{b c}, Q_{A}\right]$ observe that

$$
K_{a b}^{(+)}=-\frac{1}{2} \epsilon^{A B}\left\{\tilde{Q}_{A a}, \tilde{Q}_{B b}\right\}
$$

and so

$$
\left[K_{b c}, Q_{A a}\right]=-\ell^{-1}\left[M_{b c}, Q_{A a}\right]+\frac{1}{2} \epsilon^{B C}\left(\left[\left\{Q_{A a}, \tilde{Q}_{B b}\right\}, \tilde{Q}_{C c}\right]+\left[\left\{\tilde{Q}_{C c}, Q_{A a}\right\}, \tilde{Q}_{B b}\right]\right) .
$$

All even generators in the right-hand-side are associated with the isometries of $\mathrm{AdS}_{6}$. As a result, the right-hand-side can be found using the spinorial Lie derivatives of appendix C. This determines the commutator $\left[K_{b c}, Q_{A a}\right]$. A similar argument also determines all the other commutators of $K_{a b}$ with the remaining $Q$ 's and $\tilde{Q}$ 's. In particular all the nonvanishing commutators of even with odd generators are

$$
\begin{aligned}
{\left[V_{A B}, Q_{C \alpha}\right] } & =-\ell^{-1}\left(\epsilon_{C A} Q_{B \alpha}+\epsilon_{C B} Q_{A \alpha}\right), & {\left[\tilde{V}_{A B}, \tilde{Q}_{C \alpha}\right] } & =\ell^{-1}\left(\epsilon_{C A} \tilde{Q}_{B \alpha}+\epsilon_{C B} \tilde{Q}_{A \alpha}\right), \\
{\left[V_{A B, a}, Q_{C}\right] } & =\ell^{-1} \epsilon_{A C} \tilde{Q}_{B a}, & & {\left[V_{A B, a}, Q_{C b}\right]=\ell^{-1} \epsilon_{A C}\left(\delta_{a b} \tilde{Q}_{B}-\epsilon_{a b}{ }^{c} \tilde{Q}_{B c}\right), } \\
{\left[V_{A B, a}, \tilde{Q}_{C}\right] } & =-\ell^{-1} \epsilon_{B C} Q_{A a}, & & {\left[V_{A B, a}, \tilde{Q}_{C b}\right]=-\ell^{-1} \epsilon_{B C}\left(\delta_{a b} Q_{A}+\epsilon_{a b}{ }^{c} Q_{A c}\right), } \\
{\left[K_{a b}, Q_{A}\right] } & =-\frac{3}{2} \ell^{-1} \epsilon_{a b}{ }^{c} Q_{A c}, & {\left[K_{a b}, Q_{A c}\right] } & =\frac{3}{2} \ell^{-1} \epsilon_{a b c} Q_{A}+\frac{3}{2} \ell^{-1}\left(Q_{A a} \delta_{b c}-Q_{A b} \delta_{a c}\right), \\
{\left[M_{a b}, Q_{A}\right] } & =-\frac{1}{2} \epsilon_{a b}^{c} Q_{A c}, & {\left[M_{a b}, Q_{A c}\right] } & =\frac{1}{2} \epsilon_{a b c} Q_{A}-\frac{1}{2}\left(Q_{A a} \delta_{b c}-Q_{A b} \delta_{a c}\right), \\
{\left[K_{a b}, \tilde{Q}_{A}\right] } & =\frac{3}{2} \ell^{-1} \epsilon_{a b} \tilde{Q}_{A c}, & {\left[K_{a b}, \tilde{Q}_{A c}\right] } & =-\frac{3}{2} \ell^{-1} \epsilon_{a b c} \tilde{Q}_{A}+\frac{3}{2} \ell^{-1}\left(\tilde{Q}_{A a} \delta_{b c}-\tilde{Q}_{A b} \delta_{a c}\right), \\
{\left[M_{a b}, \tilde{Q}_{A}\right] } & =\frac{1}{2} \epsilon_{a b}^{c} \tilde{Q}_{A c}, & {\left[M_{a b}, \tilde{Q}_{A c}\right] } & =-\frac{1}{2} \epsilon_{a b c} \tilde{Q}_{A}-\frac{1}{2}\left(\tilde{Q}_{A a} \delta_{b c}-\tilde{Q}_{A b} \delta_{a c}\right),
\end{aligned}
$$

where $Q_{A \alpha}=\left(Q_{A}, Q_{A a}\right)$ and similarly for $\tilde{Q}_{A \alpha}$. The KSA is isomorphic to $\mathfrak{f}^{*}(4)$ a real form of $\mathfrak{f}(4)$ with $\mathfrak{f}^{*}(4)_{0}=\mathfrak{s o}(5,2) \oplus \mathfrak{s o}(3)$. The Lie subalgebra generated by $K_{a b}$ is isomorphic to $\mathfrak{s o}(3)$. The generators $K_{a b}$ cannot be set to zero as this will violate the super-Jacobi identities. So one expects that all these backgrounds admit an effective $\mathfrak{s o}(3)$ action.

\section{$8 \quad \mathrm{AdS}_{7}$ in $\mathrm{D}=11$ and type II theories}

To begin, a minimally supersymmetric $\mathrm{AdS}_{7}$ background admits sixteen Killing spinors and the odd generators can be identified as

$$
Q_{A}=Q_{\epsilon_{A}\left(\sigma_{+}\right)}, \quad Q_{A r}=\frac{1}{2} \omega_{r}^{(-) a b} Q_{\epsilon_{A}\left(\Gamma_{a b} \sigma_{+}\right)}, \quad \tilde{Q}_{A a}=Q_{\epsilon_{A+2}\left(\Gamma_{z a} \sigma_{+}\right)},
$$

where $\omega_{r}^{(-)}$is a basis of anti-self-dual 2-forms in $\mathbb{R}^{4}$ with

$$
\omega_{r a b}^{(-)} \omega_{s}^{(-) b}{ }_{c}=-\delta_{r s} \delta_{a c}-\epsilon_{r s}{ }^{t} \omega_{t a c}^{(-)}
$$


$\operatorname{AdS}_{k} \mathrm{KSAs}$ in $d=10$ and $d=11$

\begin{tabular}{|c|c|c|c|c|}
\hline$N$ & $\mathrm{AdS}_{4}$ & $\mathrm{AdS}_{5}$ & $\mathrm{AdS}_{6}$ & $\mathrm{AdS}_{7}$ \\
\hline 4 & $\mathfrak{o s p}(1 \mid 4)$ & - & - & - \\
\hline 8 & $\mathfrak{o s p}(2 \mid 4)$ & $\mathfrak{s l}(1 \mid 4)$ & - & - \\
\hline 12 & $\mathfrak{o s p}(3 \mid 4)$ & - & - & - \\
\hline 16 & $\mathfrak{o} \mathfrak{s p}(4 \mid 4)$ & $\mathfrak{s l}(2 \mid 4)$ & $\mathfrak{f}^{*}(4)$ & $\mathfrak{o s p}(6,2 \mid 2)$ \\
\hline 20 & $\mathfrak{o s p}(5 \mid 4)$ & - & - & - \\
\hline 24 & $\mathfrak{o s p}(6 \mid 4)$ & $\mathfrak{s l}(3 \mid 4)$ & - & - \\
\hline 28 & $\mathfrak{o} \mathfrak{s p}(7 \mid 4)$ & - & - & - \\
\hline 32 & $\mathfrak{o} \mathfrak{s p}(8 \mid 4)$ & $\mathfrak{s l}(4 \mid 4) / 1_{8 \times 8}$ & - & $\mathfrak{o s p}(6,2 \mid 4)$ \\
\hline
\end{tabular}

Table 4. In all cases, for $\operatorname{AdS}_{k}$ backgrounds $\mathfrak{g}_{0}=\mathfrak{s o}(k-1,2) \oplus \mathfrak{t}_{0} \cdot \mathfrak{f}^{*}(4)$ is a different real form to $\mathfrak{f}(4)$ which appears in the $\mathrm{AdS}_{3}$ case.

Observe that there are only 3 independent generators $Q_{A a b}$ for each $A$ as $\sigma_{+}$is restricted to satisfy ${ }^{11} \Gamma_{a b c d} \sigma_{+}=\epsilon_{a b c d} \sigma_{+}$. Computing the 1-form bilinears, we find that

$$
\begin{aligned}
\left\{Q_{A}, Q_{A^{\prime}}\right\} & =V_{A A^{\prime}}, \quad\left\{Q_{A}, Q_{B r}\right\}=\epsilon_{A B}\left(-2 \ell^{-1} M_{r}+K_{r}\right), \\
\left\{Q_{A r}, Q_{A^{\prime} s}\right\} & =4 V_{A A^{\prime}} \delta_{r s}+2 \epsilon_{A A^{\prime}} \epsilon_{r s}{ }^{t}\left(-2 \ell^{-1} M_{t}+K_{t}\right), \\
\left\{\tilde{Q}_{A a}, \tilde{Q}_{A^{\prime} a^{\prime}}\right\} & =\tilde{V}_{A A^{\prime}} \delta_{a a^{\prime}}-\epsilon_{A A^{\prime}}\left(\ell^{-1} M_{a a^{\prime}}^{(+)}+K_{a a^{\prime}}\right) \\
\left\{Q_{A}, \tilde{Q}_{B b}\right\} & =V_{A B, b}, \quad\left\{Q_{A r}, \tilde{Q}_{B b}\right\}=2 V_{A B, c} \omega_{r}^{(-) c}{ }_{b},
\end{aligned}
$$

where

$$
K_{a a^{\prime}}=2 A\left\langle\sigma_{+}, \Gamma_{z i} \Gamma_{a a^{\prime}} \sigma_{+}\right\rangle, \quad M_{r}=\frac{1}{2} \omega_{r}^{(-) a b} M_{a b}, \quad M_{a b}^{( \pm)}=M_{a b} \pm \frac{1}{2} \epsilon_{a b}^{c d} M_{c d},
$$

and

$$
V_{11, a}=-\ell^{-1} M^{-}{ }_{a}, \quad V_{12, a}=-\lambda_{a}, \quad V_{21, a}=2 \ell^{-1} M^{z}{ }_{a}+\lambda_{a}, \quad V_{22, a}=2 \ell^{-1} M^{+}{ }_{a} .
$$

The commutators of the even with the odd generators are

$$
\begin{array}{rlrl}
{\left[V_{A B}, Q_{C a}\right]} & =-\ell^{-1}\left(\epsilon_{C A} Q_{B \alpha}+\epsilon_{C B} Q_{A a}\right), \quad\left[\tilde{V}_{A B}, \tilde{Q}_{C a}\right] & =\ell^{-1}\left(\epsilon_{C A} \tilde{Q}_{B a}+\epsilon_{C B} \tilde{Q}_{A a}\right), \\
{\left[V_{A A^{\prime}, a}, Q_{B}\right]} & =\ell^{-1} \epsilon_{A B} \tilde{Q}_{A^{\prime} a}, & {\left[V_{A A^{\prime}, a}, Q_{B r}\right]} & =2 \ell^{-1} \epsilon_{A B} \omega_{r}^{(-)}{ }_{a}{ }^{b} \tilde{Q}_{A^{\prime} b}, \\
{\left[V_{A A^{\prime}, a}, \tilde{Q}_{B b}\right]} & =-\ell^{-1} \epsilon_{A^{\prime} B}\left(\delta_{a b} Q_{A}+\frac{1}{2} \omega_{a b}^{(-) r} Q_{A r}\right),\left[K_{r}, Q_{C}\right] & =-2 \ell^{-1} Q_{C r}, \\
{\left[M_{a b}, Q_{A}\right]} & =-\frac{1}{4} \omega_{r}^{(-)}{ }_{a b} Q_{A r}, \\
{\left[M_{a b}, \tilde{Q}_{A c}\right]} & =\frac{1}{2}\left(\delta_{c a} \tilde{Q}_{A b}-\delta_{A b}\right]=\omega_{r}^{(-)} \tilde{Q}_{a b} Q_{A a}+\frac{1}{2} \epsilon_{r}{ }^{s t} \omega_{s}^{(-)}{ }_{a b} Q_{A t}, \\
{\left[K_{r}, \epsilon_{a b c}{ }^{d} \tilde{Q}_{A s}\right]} & =8 \ell^{-1} Q_{A} \delta_{r s}-4 \ell^{-1} \epsilon_{r s}{ }^{t} Q_{C t}, & {\left[K_{r}, \tilde{Q}_{A a}\right]} & =-4 \ell^{-1} \omega_{r}^{(-)}{ }_{a}^{b} \tilde{Q}_{A b} .
\end{array}
$$

\footnotetext{
${ }^{11}$ One can also choose $\Gamma_{a b c d} \sigma_{+}=-\epsilon_{a b c d} \sigma_{+}$and this case can be treated in a similar way.
} 


\begin{tabular}{|c|c|c|c|c|}
\hline$N$ & $\mathrm{AdS}_{4}$ & $\mathrm{AdS}_{5}$ & $\mathrm{AdS}_{6}$ & $\mathrm{AdS}_{7}$ \\
\hline 4 & $\{0\}$ & - & - & - \\
\hline 8 & $\mathfrak{s o}(2)$ & $\mathfrak{u}(1)$ & - & - \\
\hline 12 & $\mathfrak{s o}(3)$ & - & - & - \\
\hline 16 & $\mathfrak{s o}(4)$ & $\mathfrak{u}(2)$ & $\mathfrak{s o}(3)$ & $\mathfrak{s o}(3)$ \\
\hline 20 & $\mathfrak{s o}(5)$ & - & - & - \\
\hline 24 & $\mathfrak{s o}(6)$ & $\mathfrak{u}(3)$ & - & - \\
\hline 28 & $\mathfrak{s o}(7)$ & - & - & - \\
\hline 32 & $\mathfrak{s o}(8)$ & $\mathfrak{s u}(4)$ & - & $\mathfrak{s o}(5)$ \\
\hline
\end{tabular}

Table 5. For the maximally supersymmetric $\mathrm{AdS}_{5}$ solution the isometry algebra of the internal space is $\mathfrak{s u}(4)$ instead of $\mathfrak{u}(4)$ as the $\mathfrak{u}(1)$ generator does not act effectively on the transverse 5 -sphere.

The commutators of $K_{r}$ with the odd generators have been found using a similar argument to that of the $\mathrm{AdS}_{6}$ backgrounds. The Lie algebra of the $K_{r}$ generators is $\mathfrak{s o}(3)$. The KSA is isomorphic to $\mathfrak{o s p}(6,2 \mid 2)$.

It remains to investigate the maximally supersymmetric $\mathrm{AdS}_{7}$ backgrounds. It has been shown that all such solutions are locally isometric to the $\operatorname{AdS}_{7} \times S^{4}$ background in [18]. It can be shown using the technique illustrated above that the KSA is isomorphic to $\mathfrak{o s p}(6,2 \mid 4)$. The calculation is very similar with the only difference that $\sigma_{+}$is not restricted to be (anti-) chiral with respect to the $\Gamma_{1234}$ chirality operator.

\section{Conclusions}

We have identified the KSAs, $\mathfrak{g}$, of all warped $A d S_{k} \times{ }_{w} M^{d-k}, k \geq 3$, backgrounds with the most general allowed fluxes in $d=10$ and $d=11$ dimensions, for which the even subalgebra $\mathfrak{g}_{0}$ decomposes into a direct sum of the isometries of $\mathrm{AdS}_{k}$ and those of the internal space $M^{d-k}, \mathfrak{g}_{0}=\mathfrak{s o}(k-1,2) \oplus \mathfrak{t}_{0}$. The proof utilizes (i) the solution of the KSEs for AdS backgrounds presented in $[12-14,17]$ and (ii) the closure of the KSAs demonstrated in $[15,16]$. Our results are tabulated in tables $2,3,4$ and 5.

We have demonstrated that the classification of $\mathrm{AdS}_{3} \mathrm{KSAs}$ is closely related to the classification of groups acting effectively and transitively on spheres. This is because the Lie algebra of isometries of the internal space, $\mathfrak{t}_{0}$, is associated to a group that acts transitively on a sphere in the odd subspace $\mathfrak{g}_{1}$ of the KSA. The classification of such groups is a classic problem in geometry that has been solved some time ago [10] and it has been applied in [11] to simplify the Berger classification of the holonomy groups of simply connected irreducible Riemannian manifolds. The KSAs of $\mathrm{AdS}_{3}$ backgrounds may not be simple as they can exhibit central generators. There are several potential KSAs for $\mathrm{AdS}_{3}$ backgrounds for a given number of supersymmetries $N$. For the rest of $\mathrm{AdS}_{k}, k>3$, backgrounds, we find that the KSAs are all classical and they can be uniquely characterized by the pair $(k, N)$, 
i.e. the $\mathrm{AdS}_{k}$ space under investigation and the number of supersymmetries preserved by the background.

In the context of $\mathrm{AdS}_{k} / \mathrm{CFT}_{k-1}$, for $k>3$, we have shown that the KSAs of all $\mathrm{AdS}_{k}$ backgrounds which decompose as $\mathfrak{g}_{0}=\mathfrak{s o}(k-1,2) \oplus \mathfrak{t}_{0}$ coincide with the expected superconformal algebras of field theories. So potentially all such backgrounds can have a $\mathrm{CFT}_{k-1}$ dual. The only exception perhaps is the KSA of the maximally supersymmetric $\mathrm{AdS}_{5}$ background that can exhibit a central term which however vanishes in supergravity. In $\mathrm{AdS}_{3}$ backgrounds, there are many KSAs that arise for a given number of supersymmetries $N$ and can exhibit one or more central generators. The role of these central terms should be clarified in both supergravity and in quantum theory.

Our results have applications in the classification of supersymmetric AdS backgrounds. If AdS backgrounds preserve more than 16 supersymmetries, $N>16$, then under some mild assumptions it can be shown that the warp factor is constant and therefore they are products $A d S_{k} \times M^{d-k}$. Furthermore they must be homogenous [19] and so $M^{d-k}=G / K$. The identification of all KSAs of $\mathrm{AdS}_{k}$ backgrounds allows one to set $\mathfrak{L} \mathfrak{i} \in=\mathfrak{t}_{0}$. As all the Lie algrebras $\mathfrak{t}_{0}$ of isometries of the internal spaces are known, all the internal spaces can be identified as homogenous spaces of groups with Lie algebra $\mathfrak{t}_{0}$. So far, the $\operatorname{AdS}_{k}$ backgrounds that preserve more than 16 supersymmetries have been classified for $k=4$ [20] and $k=5$ [21]. In the former case the classification of the KSAs for $\mathrm{AdS}_{4}$ backgrounds has been utilized in an essential way. For $k>5, \operatorname{AdS}_{k}$ backgrounds preserve either 16 or 32 supersymmetries and so for $N>16$ are included in the classification of maximal supersymmetric backgrounds in [18].

For $\mathrm{AdS}_{k}$ backgrounds that preserve 16 or less supersymmetries, $N \leq 16$, the KSA may not act transitively on the internal space. Further progress on the classification of such backgrounds will require a detailed analysis of the orbits of the KSAs in the internal spaces as presented in [24] for $\mathrm{AdS}_{6}$ backgrounds. Investigations of the geometry of such AdS backgrounds based on superalgebra considerations have been made before, see e.g. [22, 23]. However now this can be done more systematically as all possibilities have been identified.

\section{Acknowledgments}

GP wishes to thank Jose Figueroa-O'Farrill and Alessandro Tomasiello for many helpful discussions. UG and GP would like to thank MITP for providing a stimulating environment during the workshop "Geometry, Gravity and Supersymmetry" where part of this project was completed. GP is partially supported from the STFC rolling grant ST/J002798/1. JG is supported by the STFC Consolidated Grant ST/L000490/1. UG is supported by the Swedish Research Council.

Data management. No data beyond those presented and cited in this work are needed to validate this study. 


\section{A Invariance of (massive) IIA fluxes}

In this appendix we will give a proof to the statement that the Killing vector bilinears leave invariant all the fields of (massive) IIA supergravity, i.e. they are Killing vectors and preserve all the fluxes. The proof will rely on the Killing spinor equations

$$
\begin{aligned}
\mathcal{D}_{M} \epsilon \equiv & \nabla_{M} \epsilon+\frac{1}{8} H_{M P_{1} P_{2}} \Gamma^{P_{1} P_{2}} \Gamma_{11} \epsilon+\frac{1}{8} e^{\Phi} \tilde{S} \Gamma_{M} \epsilon \\
& +\frac{1}{16} e^{\Phi} \tilde{F}_{P_{1} P_{2}} \Gamma^{P_{1} P_{2}} \Gamma_{M} \Gamma_{11} \epsilon+\frac{1}{8 \cdot 4 !} e^{\Phi} \tilde{G}_{P_{1} \cdots P_{4}} \Gamma^{P_{1} \cdots P_{4}} \Gamma_{M} \epsilon
\end{aligned}
$$

and

$$
\begin{aligned}
\mathcal{A} \epsilon \equiv & \partial_{P} \Phi \Gamma^{P} \epsilon+\frac{1}{12} H_{P_{1} P_{2} P_{3}} \Gamma^{P_{1} P_{2} P_{3}} \Gamma_{11} \epsilon+\frac{5}{4} e^{\Phi} \tilde{S} \epsilon \\
& +\frac{3}{8} e^{\Phi} \tilde{F}_{P_{1} P_{2}} \Gamma^{P_{1} P_{2}} \Gamma_{11} \epsilon+\frac{1}{4 \cdot 4 !} e^{\Phi} \tilde{G}_{P_{1} \cdots P_{4}} \Gamma^{P_{1} \cdots P_{4}} \epsilon,
\end{aligned}
$$

where $\nabla$ is the spin connection, $H$ is the NS-NS 3 -form field strength, $\tilde{S}, \tilde{F}, \tilde{G}$ are the RR $k$-form field strengths, for $k=0,2,4$ respectively, and $\Phi$ is the dilaton. For later convenience, we set

$$
S=e^{\Phi} \tilde{S}, \quad F=e^{\Phi} \tilde{F}, \quad G=e^{\Phi} \tilde{G}
$$

In addition to the Killing spinor equations the proof will also rely on the field equations and Bianchi identities (for relevant expressions in the conventions introduced above see [25]), and the result will thus hold in general for all supersymmetric supergravity solutions.

It is convenient to introduce the following notation

$$
\begin{aligned}
\alpha_{B_{1} \cdots B_{k}}^{I J} & \equiv B\left(\epsilon^{I}, \Gamma_{B_{1} \cdots B_{k}} \epsilon^{J}\right), \\
\tau_{B_{1} \cdots B_{k}}^{I J} & \equiv B\left(\epsilon^{I}, \Gamma_{B_{1} \cdots B_{k}} \tilde{\epsilon}^{J}\right),
\end{aligned}
$$

where $\tilde{\epsilon}=\Gamma_{11} \epsilon$, the inner product $B\left(\epsilon^{I}, \epsilon^{J}\right) \equiv\left\langle\Gamma_{0} C * \epsilon^{I}, \epsilon^{J}\right\rangle$, where $C=\Gamma_{6789}$, is antisymmetric, i.e. $B\left(\epsilon^{I}, \epsilon^{J}\right)=-B\left(\epsilon^{J}, \epsilon^{I}\right)$ and all $\Gamma$-matrices are anti-Hermitian with respect to this inner product, i.e. $B\left(\Gamma_{A} \epsilon^{I}, \epsilon^{J}\right)=-B\left(\epsilon^{I}, \Gamma_{A} \epsilon^{J}\right)$.

Denoting $\alpha_{B_{1} \cdots B_{k}}^{I J}=\alpha_{(k)}^{I J}$ and $\tau_{B_{1} \cdots B_{k}}^{I J}=\tau_{(k)}^{I J}$ the bilinears have the symmetry properties

$$
\begin{array}{rlrl}
\alpha_{(k)}^{I J} & =\alpha_{(k)}^{J I} & k & =1,2,5 \\
\alpha_{(k)}^{I J} & =-\alpha_{(k)}^{J I} & k & =0,3,4
\end{array}
$$

and

$$
\begin{array}{rlrl}
\tau_{(k)}^{I J} & =\tau_{(k)}^{J I} & k & =0,1,4,5 \\
\tau_{(k)}^{I J} & =-\tau_{(k)}^{J I} & k & =2,3 .
\end{array}
$$

First we verify that there is a set of 1 -form bi-linears whose associated vectors are Killing. We write the gravitino KSE as

$$
\left(\nabla_{A}+\Sigma_{A}\right) \epsilon=0
$$


where

$$
\begin{aligned}
\Sigma_{A}= & \frac{1}{8} H_{M P_{1} P_{2}} \Gamma^{P_{1} P_{2}} \Gamma_{11}+\frac{1}{8} S \Gamma_{M}+\frac{1}{16} F_{P_{1} P_{2}} \Gamma^{P_{1} P_{2}} \Gamma_{M} \Gamma_{11} \\
& +\frac{1}{8 \cdot 4 !} G_{P_{1} \cdots P_{4}} \Gamma^{P_{1} \cdots P_{4}} \Gamma_{M},
\end{aligned}
$$

which we use to replace covariant derivatives with fluxes and $\Gamma$-matrixes. The 1 -form bilinears associated with the Killing vectors are $\alpha_{A}^{I J} e^{A}$, which we see by computing

$$
\begin{aligned}
\nabla_{A} \alpha_{B}^{I J}= & \nabla_{A} B\left(\epsilon^{I}, \Gamma_{B} \epsilon^{J}\right) \\
= & B\left(\nabla_{A} \epsilon^{I}, \Gamma_{B} \epsilon^{J}\right)+B\left(\epsilon^{I}, \Gamma_{B} \nabla_{A} \epsilon^{J}\right) \\
= & -B\left(\Sigma_{A} \epsilon^{I}, \Gamma_{B} \epsilon^{J}\right)-B\left(\epsilon^{I}, \Gamma_{B} \Sigma_{A} \epsilon^{J}\right) \\
= & B\left(\Gamma_{B} \epsilon^{J}, \Sigma_{A} \epsilon^{I}\right)-B\left(\epsilon^{I}, \Gamma_{B} \Sigma_{A} \epsilon^{J}\right) \\
= & -B\left(\epsilon^{J}, \Gamma_{B} \Sigma_{A} \epsilon^{I}\right)-B\left(\epsilon^{I}, \Gamma_{B} \Sigma_{A} \epsilon^{J}\right) \\
= & -2 B\left(\epsilon^{(I}, \Gamma_{B} \Sigma_{A} \epsilon^{J)}\right) \\
= & \left(\frac{1}{4} S \alpha_{A B}^{I J}+\frac{1}{8} G_{A B}{ }^{C_{1} C_{2}} \alpha_{C_{1} C_{2}}^{I J}+\frac{1}{96} G^{C_{1} C_{2} C_{3} C_{4}} \alpha_{A B C_{1} C_{2} C_{3} C_{4}}^{I J}\right. \\
& \left.+\frac{1}{4} F_{A B} \tau^{I J}-\frac{1}{2} H_{A B} C_{C}^{I J}+\frac{1}{8} F^{C_{1} C_{2}} \tau_{A B C_{1} C_{2}}^{I J}\right) .
\end{aligned}
$$

Since the resulting expression is antisymmetric in its free indices we find that $\nabla_{\left({ }_{A}\right.} \alpha_{B)}^{I J}=0$ and hence the vectors associated with $\alpha_{A}^{I J} e^{A}$ are Killing.

Note that the dilatino KSE (A.2) imply that

$$
0=B\left(\epsilon^{(I}, \mathcal{A} \epsilon^{J)}\right)=\alpha_{A}^{I J} \partial^{A} \Phi,
$$

and hence $i_{K} d \Phi=0$, where $K=\alpha_{A}^{I J} e^{A}$ denotes the 1-forms associated with the Killing vectors with the $I J$ indices suppressed. With this relation it follows that the Killing vectors preserve the dilaton:

$$
\mathcal{L}_{K} \Phi:=i_{K} d \Phi+d\left(i_{K} \Phi\right)=0
$$

since $i_{K} \Phi \equiv 0$.

To see that the 3-form flux $H$ is preserved we need to analyse the 1-form bi-linears which are not related to the Killing vectors, i.e. $\tau_{A}^{I J} e^{A}$. As above, we find that

$$
\begin{aligned}
\nabla_{[A} \tau_{B]}^{I J}= & -2 B\left(\epsilon^{(I}, \Gamma_{11} \Gamma_{[A} \Sigma_{B]} \epsilon^{J)}\right) \\
= & -\frac{1}{2} H_{A B}{ }^{C} \alpha_{C}^{I J}
\end{aligned}
$$

or equivalently

$$
d \tau_{(1)}^{I J}=-i_{K} H,
$$

where we have indicated the degree of the form $\tau$ and suppressed the indices labelling the Killing spinors. By taking the exterior derivative of (A.13), and using the Bianchi identity for $H$, i.e. $d H=0$, it follows that

$$
\mathcal{L}_{K} H=0
$$

and hence the Killing vectors preserve also the $H$ flux. 
We now turn to the 2-form flux $F$. Computing the (covariant) derivative of the scalar $\tau^{I J}$, and making use of the gravitino KSE as above, we find

$$
d \tau_{(0)}^{I J}=i_{K} F+d \Phi \tau_{(0)}^{I J}-S \tau_{(1)}^{I J} .
$$

Acting with another derivative on (A.15), and re-substituting (A.15) into the resulting expression, we obtain

$$
0=\mathcal{L}_{K} F-i_{K}(d F)-d \Phi \wedge i_{K} F-S d \tau_{(1)}^{I J}=\mathcal{L}_{K} F,
$$

where in the second step we have used (A.13) and the Bianchi identity for $F$, i.e.

$$
0=d F-d \Phi \wedge F-S H .
$$

This shows that the $F$ flux is preserved.

For the $G$ flux a similar analysis can be preformed. Computing the covariant derivative of $\alpha_{(2)}^{I J}$ leads to

$$
d \alpha_{(2)}^{I J}=i_{K} G+d \Phi \wedge \alpha_{(2)}^{I J}+H \tau_{(0)}^{I J}-F \wedge \tau_{(1)}^{I J} .
$$

Acting with an exterior derivative on (A.18) and re-substituting (A.18) into the resulting expression, and using (A.13), (A.15) and (A.17), we obtain

$$
0=\mathcal{L}_{K} G-i_{K}(d G)-d \Phi \wedge i_{K} G+i_{K} F \wedge H+F \wedge i_{K} H=\mathcal{L}_{K} G,
$$

where in the second step we have used the Bianchi identity for $G$, i.e.

$$
0=d G-d \Phi \wedge G-F \wedge H
$$

concluding the proof of the preservation of $G$.

Finally, $\mathcal{L}_{K} S=0$ follows from the constancy of the Romans mass parameter $\tilde{S}$, which completes the proof. For the computations in this appendix the Mathematica package GAMMA [26] has been used.

\section{B AdS superalgebra}

Here we collect some of the key formulae that are needed to determine the superalgebras of AdS backgrounds. The proof we have presented relies on the observation that the commutators of the superalgebra can be computed explicitly when the generators are associated with symmetries of the AdS subspace of the background. For this we give the Killing vectors of AdS subspace and their commutators as well as their action on the Killing spinors.

\section{B.1 Isometries of AdS}

The associated 1-forms of the Killing vectors along the AdS subspace of $\operatorname{AdS}_{k} \times_{w} M^{k}$, $n \geq 3$ equipped with the metric (2.3) are

$$
\begin{array}{rlrlrl}
\lambda^{+} & =A^{2} e^{2 z / \ell} \mathbf{e}^{+}, & \lambda^{-} & =\mathbf{e}^{-}, & \lambda^{a} & =A e^{z / \ell} \mathbf{e}^{a}, \\
\lambda^{z} & =A \mathbf{e}^{z}-\ell^{-1} \hat{r} \lambda^{+}-\ell^{-1} u \lambda^{-}-\ell^{-1} x_{a} \lambda^{a}, & M^{+-} & =\hat{r} \lambda^{+}-u \lambda^{-}, \\
M^{+a} & =x^{a} \lambda^{+}-u \lambda^{a}, & M^{-a}=x^{a} \lambda^{-}-\hat{r} \lambda^{a}, & M^{a b} & =x^{b} \lambda^{a}-x^{a} \lambda^{b}, \\
M^{z+} & =p \lambda^{+}+u \lambda^{z}, & M^{z-}=p \lambda^{-}+\hat{r} \lambda^{z}, & M^{z a} & =p \lambda^{a}+x^{a} \lambda^{z},
\end{array}
$$


where $p=\frac{1}{2}\left(2 \ell^{-1} u \hat{r}+\ell e^{-2 z / \ell}-\ell+\ell^{-1} \mathbf{x}^{2}\right)$ and $\hat{r}=r A^{-2} e^{-2 z / \ell}$. Note that $\ell$ is the radius of $\operatorname{AdS}_{k}$, the warp factor, $A$, depends only on the coordinates $y^{i}$ of $M^{k}$ while $u, r, z, x^{a}$ for $a=1, \ldots, n-3$ are the coordinates of $\mathrm{AdS}_{k}$ subspace, and $g_{i j}$ is the metric on $M^{k}$.

The associated Killing vectors are

$$
\begin{aligned}
& \kappa^{+}=A^{2} e^{2 z / \ell} \partial_{r}, \quad \kappa^{-}=\partial_{u}, \quad \kappa^{a}=\partial_{a}, \\
& \kappa^{z}=\partial_{z}+\ell^{-1} r \partial_{r}-\ell^{-1} u \partial_{u}-\ell^{-1} x^{a} \partial_{a}, \quad N^{+-}=r \partial_{r}-u \partial_{u}, \\
& N^{+a}=A^{2} e^{2 z / \ell} x^{a} \partial_{r}-u \partial_{a}, \quad N^{-a}=x^{a} \partial_{u}-A^{-2} e^{-2 z / \ell} r \partial_{a}, \quad N^{a b}=x^{b} \partial_{a}-x^{a} \partial_{b}, \\
& N^{z+}=A^{2} e^{2 z / \ell} p \partial_{r}+u \kappa^{z}, \quad N^{z-}=p \partial_{u}+A^{-2} e^{-2 z / \ell} r \kappa^{z}, \quad N^{z a}=p \partial_{a}+x^{a} \kappa^{z} .
\end{aligned}
$$

Moreover, the commutators of these Killing vectors are

$$
\begin{aligned}
{\left[\lambda^{\mu}, \lambda^{\nu}\right] } & =\ell^{-1} f^{\mu \nu}{ }_{\kappa} \lambda^{\kappa}, \quad\left[\lambda^{\mu}, M^{\nu \sigma}\right]=-2 \eta^{\mu[\nu} \lambda^{\sigma]}+\ell^{-1} f_{\kappa_{1} \kappa_{2}}^{\mu \nu \sigma} M^{\kappa_{1} \kappa_{2}}, \\
{\left[M^{\mu \nu}, M^{\sigma \tau}\right] } & =2\left(\eta^{\mu[\tau} M^{\sigma] \nu}-\eta^{\nu[\tau} M^{\sigma] \mu}\right),
\end{aligned}
$$

where

$$
\begin{aligned}
& f_{ \pm}^{z \pm}=1, \quad f_{b}^{z a}=\delta_{b}^{a}, \quad f^{ \pm \mp z}{ }_{[+-]}= \pm \frac{1}{2}, \quad f_{[ \pm b]}^{a \pm z}=-\frac{1}{2} \delta_{b}^{a} \\
& f_{c d}^{a z b}=-\delta_{[c}^{a} \delta_{d]}^{b}, \quad f_{[ \pm z]}^{z \pm z}=-\frac{1}{2}, \quad f_{[z b]}^{z z a}=-\frac{1}{2} \delta_{b}^{a}, \quad f_{ \pm b}^{ \pm z a}=-\frac{1}{2} \delta^{a}{ }_{b} .
\end{aligned}
$$

The Lie algebra is isomorphic to $\mathfrak{s o}(n-1,2)$ as expected.

\section{B.2 Spinorial Lie derivatives along AdS}

The commutators of the even generators associated with the isometries of AdS and odd generators associated with the Killing spinors of the superalgebra can also be explicitly found via the spinorial derivative (2.2) of the isometries of AdS on the Killing spinors of the backgrounds. In particular, one has

$$
\begin{aligned}
\mathcal{L}_{\lambda^{+}} \epsilon= & A^{2} e^{2 z / \ell} \partial_{r} \epsilon, \quad \mathcal{L}_{\lambda^{-}} \epsilon=\partial_{u} \epsilon, \quad \mathcal{L}_{\lambda^{a}} \epsilon=\partial_{a} \epsilon \\
\mathcal{L}_{\lambda^{z}} \epsilon= & \left(\partial_{z}+\ell^{-1} r \partial_{r}-\ell^{-1} u \partial_{u}-\ell^{-1} x^{a} \partial_{a}\right) \epsilon+\frac{1}{2} \ell^{-1} \Gamma_{+-} \epsilon \\
\mathcal{L}_{M^{+-}} \epsilon= & \left(r \partial_{r}-u \partial_{u}\right) \epsilon+\frac{1}{2} \Gamma_{+-} \epsilon \\
\mathcal{L}_{M^{+a}} \epsilon= & \left(x_{a} A^{2} e^{2 z / \ell} \partial_{r}-u \partial_{a}\right) \epsilon-\frac{1}{2} A e^{z / \ell} \Gamma_{-a} \epsilon \\
\mathcal{L}_{M^{-a}} \epsilon= & \left(x_{a} \partial_{u}-\hat{r} \partial_{a}\right) \epsilon-\frac{1}{2} A^{-1} e^{-z / \ell} \Gamma_{+a} \epsilon \\
\mathcal{L}_{M^{a b}} \epsilon= & \left(x_{b} \partial_{a}-x_{a} \partial_{b}\right) \epsilon-\frac{1}{2} \Gamma_{a b} \epsilon \\
\mathcal{L}_{M^{z+}} \epsilon= & {\left[\left(p+\ell^{-1} u \hat{r}\right) A^{2} e^{2 z / \ell} \partial_{r}+u \partial_{z}-\ell^{-1} u^{2} \partial_{u}-\ell^{-1} u x^{a} \partial_{a}\right] \epsilon } \\
& +\left(\frac{1}{2} A \Gamma_{-z}+\ell^{-1} u \Gamma_{+-}-\frac{1}{2} \ell^{-1} A e^{z / \ell} x^{a} \Gamma_{-a}\right) \epsilon \\
\mathcal{L}_{M^{z-}} \epsilon= & {\left[\left(p-\ell^{-1} u \hat{r}\right) \partial_{u}+\hat{r} \partial_{z}+\ell^{-1} r \hat{r} \partial_{r}-\ell^{-1} \hat{r} x^{a} \partial_{a}\right] \epsilon } \\
& +\left(\frac{1}{2} A^{-1} e^{-2 z / \ell} \Gamma_{+z}-\frac{1}{2} \ell^{-1} A^{-1} e^{-z / \ell} x^{a} \Gamma_{+a}\right) \epsilon
\end{aligned}
$$




$$
\begin{aligned}
\mathcal{L}_{M^{z a}} \epsilon= & {\left[\left(p \delta_{a}^{b}-\ell^{-1} x_{a} x^{b}\right) \partial_{b}+x_{a} \partial_{z}+\ell^{-1} r x_{a} \partial_{r}-\ell^{-1} u x_{a} \partial_{u}\right] \epsilon } \\
& +\left(\frac{1}{2} \ell^{-1} x_{a} \Gamma_{+-}+\frac{1}{2} \ell^{-1} u A^{-1} e^{-z / \ell} \Gamma_{+a}\right. \\
& \left.+\frac{1}{2} \ell^{-1} r A^{-1} e^{-z / \ell} \Gamma_{-a}-\frac{1}{2} e^{-z / \ell} \Gamma_{z a}-\frac{1}{2} \ell^{-1} x^{b} \Gamma_{a b}\right) \epsilon
\end{aligned}
$$

These spinorial Lie derivatives determine all such commutators as the dependence of the Killing spinors on the AdS coordinates is known. The super-Jacobi identities are then used to restrict the remaining commutators of the even generators associated with the isometries of the internal space with odd generators of the superalgebra.

\section{Commutators}

\section{C.1 1-form bilinears}

The linearly independent Killing spinors of $\operatorname{AdS}_{k}, n \geq 3$, backgrounds expressed in terms of $\sigma_{ \pm}$and $\tau_{ \pm}$are

$$
\begin{aligned}
& \epsilon_{1}\left(\sigma_{+}\right)=\sigma_{+}, \quad \epsilon_{2}\left(\sigma_{-}\right)=\sigma_{-}-\ell^{-1} e^{\frac{z}{\ell}} x^{a} \Gamma_{a z} \sigma_{-}-\ell^{-1} A^{-1} u \Gamma_{+z} \sigma_{-} \\
& \epsilon_{3}\left(\tau_{+}\right)=e^{-\frac{z}{\ell}} \tau_{+}-\ell^{-1} A^{-1} r e^{-\frac{z}{\ell}} \Gamma_{-z} \tau_{+}-\ell^{-1} x^{a} \Gamma_{a z} \tau_{+}, \quad \epsilon_{4}\left(\tau_{-}\right)=e^{\frac{z}{\ell}} \tau_{-} .
\end{aligned}
$$

For $n=3$, the terms proportional to $x^{a}$ do not occur and they should be set to zero.

The anti-commutator of the odd generators can be founds from the 1-form bilinears.

These are

$$
\begin{aligned}
& K\left(\epsilon_{1}, \epsilon_{1}\right)=2\left\|\sigma_{+}\right\|^{2} \mathbf{e}^{-}, \\
& K\left(\epsilon_{2}, \epsilon_{2}\right)=-2\left(1+\ell^{-2} x^{2} e^{\frac{2 z}{\ell}}\right)\left\|\sigma_{-}\right\|^{2} \mathbf{e}^{+}+4 \ell^{-2} A^{-2} u^{2}\left\|\sigma_{-}\right\|^{2} \mathbf{e}^{-} \\
& -4 \ell^{-1} A^{-1} u\left\|\sigma_{-}\right\|^{2} \mathbf{e}^{z}+4 \ell^{-2} A^{-1} e^{\frac{z}{\ell}} u x_{a}\left\|\sigma_{-}\right\|^{2} \mathbf{e}^{a} \\
& K\left(\epsilon_{3}, \epsilon_{3}\right)=-4 \ell^{-2} A^{-2} r^{2} e^{-\frac{2 z}{\ell}}\left\|\tau_{+}\right\|^{2} \mathbf{e}^{+}+2\left(e^{-\frac{2 z}{\ell}}+\ell^{-2} x^{2}\right)\left\|\tau_{+}\right\|^{2} \mathbf{e}^{-} \\
& +4 \ell^{-1} A^{-1} r e^{-\frac{2 z}{\ell}}\left\|\tau_{+}\right\|^{2} \mathbf{e}^{z}-4 \ell^{-2} A^{-1} r e^{-\frac{z}{\ell}} x_{a}\left\|\tau_{+}\right\|^{2} \mathbf{e}^{a} \\
& K\left(\epsilon_{4}, \epsilon_{4}\right)=-2 e^{\frac{2 z}{\ell}}\left\|\tau_{-}\right\|^{2} \mathbf{e}^{+}, \\
& K\left(\epsilon_{2}, \epsilon_{1}\right)=-2 \ell^{-1} A^{-1} u\left\langle\Gamma_{+z} \sigma_{-}, \sigma_{+}\right\rangle \mathbf{e}^{-}+\left[\left\langle\Gamma_{+z} \sigma_{-}, \sigma_{+}\right\rangle-\ell^{-1} e^{\frac{z}{\ell}} x^{a}\left\langle\Gamma_{+} \sigma_{-}, \Gamma_{a} \sigma_{+}\right\rangle\right] \mathbf{e}^{z} \\
& -\left[\left\langle\Gamma_{+} \sigma_{-}, \Gamma_{c} \sigma_{+}\right\rangle+\ell^{-1} e^{\frac{z}{\ell}} x^{a}\left\langle\Gamma_{+z} \sigma_{-}, \Gamma_{a} \Gamma_{c} \sigma_{+}\right\rangle\right] \mathbf{e}^{c} \\
& -\left[\left\langle\Gamma_{+} \sigma_{-}, \Gamma_{i} \sigma_{+}\right\rangle+\ell^{-1} e^{\frac{z}{\ell}} x^{a}\left\langle\Gamma_{+z} \sigma_{-}, \Gamma_{a} \Gamma_{i} \sigma_{+}\right\rangle\right] \mathbf{e}^{i} \\
& K\left(\epsilon_{3}, \epsilon_{1}\right)=2\left(e^{-\frac{z}{\ell}}\left\langle\tau_{+}, \sigma_{+}\right\rangle-\ell^{-1} x^{a}\left\langle\Gamma_{a z} \tau_{+}, \sigma_{+}\right\rangle\right) \mathbf{e}^{-} \\
& +2 \ell^{-1} A^{-1} r e^{-\frac{z}{\ell}}\left\langle\tau_{+}, \sigma_{+}\right\rangle \mathbf{e}^{z}+2 \ell^{-1} A^{-1} r e^{-\frac{z}{\ell}}\left\langle\tau_{+}, \Gamma_{z a} \sigma_{+}\right\rangle \mathbf{e}^{a} \\
& +2 \ell^{-1} A^{-1} r e^{-\frac{z}{\ell}}\left\langle\tau_{+}, \Gamma_{z i} \sigma_{+}\right\rangle \mathbf{e}^{i} \\
& K\left(\epsilon_{4}, \epsilon_{1}\right)=-e^{\frac{z}{\ell}}\left\langle\Gamma_{+} \tau_{-}, \Gamma_{z} \sigma_{+}\right\rangle \mathbf{e}^{z}-e^{\frac{z}{\ell}}\left\langle\Gamma_{+} \tau_{-}, \Gamma_{a} \sigma_{+}\right\rangle \mathbf{e}^{a}-e^{\frac{z}{\ell}}\left\langle\Gamma_{+} \tau_{-}, \Gamma_{i} \sigma_{+}\right\rangle \mathbf{e}^{i} \\
& K\left(\epsilon_{2}, \epsilon_{3}\right)=2 \ell^{-1} A^{-1} r\left[-e^{-\frac{z}{\ell}}\left\langle\Gamma_{+z} \sigma_{-}, \tau_{+}\right\rangle+\ell^{-1} x^{a}\left\langle\Gamma_{+} \sigma_{-}, \Gamma_{a} \tau_{+}\right\rangle\right] \mathbf{e}^{+} \\
& +2 \ell^{-1} A^{-1} u\left[-e^{-\frac{z}{\ell}}\left\langle\Gamma_{+z} \sigma_{-}, \tau_{+}\right\rangle+\ell^{-1} x^{a}\left\langle\Gamma_{+} \sigma_{-}, \Gamma_{a} \tau_{+}\right\rangle\right] \mathbf{e}^{-} \\
& +\left[e^{-\frac{z}{\ell}}\left\langle\Gamma_{+z} \sigma_{-}, \tau_{+}\right\rangle-2 \ell^{-1} x^{a}\left\langle\Gamma_{+} \sigma_{-}, \Gamma_{a} \tau_{+}\right\rangle-\ell^{-2} e^{\frac{z}{\ell}} x^{2}\left\langle\Gamma_{+z} \sigma_{-}, \tau_{+}\right\rangle\right. \\
& \left.-2 \ell^{-2} A^{-2} u r e^{-\frac{z}{\ell}}\left\langle\Gamma_{+z} \sigma_{-}, \tau_{+}\right\rangle\right] \mathbf{e}^{z} \\
& +\left[-e^{-\frac{z}{\ell}}\left\langle\Gamma_{+} \sigma_{-}, \Gamma_{c} \tau_{+}\right\rangle-2 \ell^{-1} x_{c}\left\langle\Gamma_{+z} \sigma_{-}, \tau_{+}\right\rangle+\ell^{-2} e^{\frac{z}{\ell}} x^{a} x^{b}\left\langle\Gamma_{+} \sigma_{-}, \Gamma_{a} \Gamma_{c} \Gamma_{b} \tau_{+}\right\rangle\right. \\
& \left.-2 \ell^{-2} A^{-2} u r e^{-\frac{z}{\ell}}\left\langle\Gamma_{+} \sigma_{-}, \Gamma_{c} \tau_{+}\right\rangle\right] \mathbf{e}^{c} \\
& -\left[e^{-\frac{z}{\ell}}+\ell^{-2} e^{\frac{z}{\ell}} x^{2}+2 \ell^{-2} A^{-2} u r e^{-\frac{z}{\ell}}\right]\left\langle\Gamma_{+} \sigma_{-}, \Gamma_{i} \tau_{+}\right\rangle \mathbf{e}^{i}
\end{aligned}
$$




$$
\begin{aligned}
K\left(\epsilon_{2}, \epsilon_{4}\right)= & -2\left[e^{\frac{z}{\ell}}\left\langle\sigma_{-}, \tau_{-}\right\rangle-\ell^{-1} e^{\frac{2 z}{\ell}} x^{a}\left\langle\sigma_{-}, \Gamma_{z a} \tau_{-}\right\rangle\right] \mathbf{e}^{+}-2 \ell^{-1} A^{-1} u e^{\frac{z}{\ell}}\left\langle\sigma_{-}, \tau_{-}\right\rangle \mathbf{e}^{z} \\
& -2 \ell^{-1} A^{-1} u e^{\frac{z}{\ell}}\left\langle\sigma_{-}, \Gamma_{z a} \tau_{-}\right\rangle \mathbf{e}^{a}-2 \ell^{-1} A^{-1} u e^{\frac{z}{\ell}}\left\langle\sigma_{-}, \Gamma_{z i} \tau_{-}\right\rangle \mathbf{e}^{i} \\
K\left(\epsilon_{3}, \epsilon_{4}\right)= & -2 \ell^{-1} r A^{-1}\left\langle\tau_{+}, \Gamma_{+z} \tau_{-}\right\rangle \mathbf{e}^{+}+\left[\left\langle\tau_{+}, \Gamma_{+z} \tau_{-}\right\rangle+\ell^{-1} x^{a} e^{\frac{z}{\ell}}\left\langle\tau_{+}, \Gamma_{+a} \tau_{-}\right\rangle\right] \mathbf{e}^{z} \\
& +\left[\left\langle\tau_{+}, \Gamma_{+c} \tau_{-}\right\rangle-\ell^{-1} x^{a} e^{\frac{z}{\ell}}\left\langle\tau_{+}, \Gamma_{a} \Gamma_{c} \Gamma_{+z} \tau_{-}\right\rangle\right] \mathbf{e}^{c} \\
& +\left[\left\langle\tau_{+}, \Gamma_{+i} \tau_{-}\right\rangle-\ell^{-1} x^{a} e^{\frac{z}{\ell}}\left\langle\tau_{+}, \Gamma_{a} \Gamma_{i} \Gamma_{+z} \tau_{-}\right\rangle\right] \mathbf{e}^{i} .
\end{aligned}
$$

Note that bilinears of different Killing spinors $\epsilon^{r}$ are given as above after replacing $\left\|\sigma_{+}\right\|^{2}$ with the inner product $\left\langle\sigma_{+}^{r}, \sigma_{+}^{s}\right\rangle$ and similarly for the rest of the $\sigma_{ \pm}$and $\tau_{ \pm}$spinors.

After imposing the conditions that arise from the global considerations, the form of the bilinears simplifies to

$$
\begin{aligned}
K\left(\epsilon_{1}, \epsilon_{1}\right)= & 2\left\|\sigma_{+}\right\|^{2} \mathbf{e}^{-} \\
K\left(\epsilon_{2}, \epsilon_{2}\right)= & -2\left(1+\ell^{-2} x^{2} e^{\frac{2 z}{\ell}}\right)\left\|\sigma_{-}\right\|^{2} \mathbf{e}^{+}+4 \ell^{-2} A^{-2} u^{2}\left\|\sigma_{-}\right\|^{2} \mathbf{e}^{-} \\
& -4 \ell^{-1} A^{-1} u\left\|\sigma_{-}\right\|^{2} \mathbf{e}^{z}+4 \ell^{-2} A^{-1} e^{\frac{z}{\ell}} u x_{a}\left\|\sigma_{-}\right\|^{2} \mathbf{e}^{a} \\
K\left(\epsilon_{3}, \epsilon_{3}\right)= & -4 \ell^{-2} A^{-2} r^{2} e^{-\frac{2 z}{\ell}}\left\|\tau_{+}\right\|^{2} \mathbf{e}^{+}+2\left(e^{-\frac{2 z}{\ell}}+\ell^{-2} x^{2}\right)\left\|\tau_{+}\right\|^{2} \mathbf{e}^{-} \\
& +4 \ell^{-1} A^{-1} r e^{-\frac{2 z}{\ell}}\left\|\tau_{+}\right\|^{2} \mathbf{e}^{z}-4 \ell^{-2} A^{-1} r e^{-\frac{z}{\ell}} x_{a}\left\|\tau_{+}\right\|^{2} \mathbf{e}^{a} \\
K\left(\epsilon_{4}, \epsilon_{4}\right)= & -2 e^{\frac{2 z}{\ell}}\left\|\tau_{-}\right\|^{2} \mathbf{e}^{+} \\
K\left(\epsilon_{2}, \epsilon_{1}\right)= & -2 \ell^{-1} A^{-1} u\left\langle\Gamma_{+z} \sigma_{-}, \sigma_{+}\right\rangle \mathbf{e}^{-}+\left\langle\Gamma_{+z} \sigma_{-}, \sigma_{+}\right\rangle \mathbf{e}^{z} \\
& -\ell^{-1} e^{\frac{z}{\ell}} x^{a}\left\langle\Gamma_{+z} \sigma_{-}, \Gamma_{a} \Gamma_{c} \sigma_{+}\right\rangle \mathbf{e}^{c}-\left\langle\Gamma_{+} \sigma_{-}, \Gamma_{i} \sigma_{+}\right\rangle \mathbf{e}^{i} \\
K\left(\epsilon_{3}, \epsilon_{1}\right)= & -2 \ell^{-1} x^{a}\left\langle\Gamma_{a z} \tau_{+}, \sigma_{+}\right\rangle \mathbf{e}^{-}+2 \ell^{-1} A^{-1} r e^{-\frac{z}{\ell}}\left\langle\tau_{+}, \Gamma_{z a} \sigma_{+}\right\rangle \mathbf{e}^{a} \\
K\left(\epsilon_{4}, \epsilon_{1}\right)= & -e^{\frac{z}{\ell}}\left\langle\Gamma_{+} \tau_{-}, \Gamma_{a} \sigma_{+}\right\rangle \mathbf{e}^{a} \\
K\left(\epsilon_{2}, \epsilon_{3}\right)= & 2 \ell^{-2} A^{-1} r x^{a}\left\langle\Gamma_{+} \sigma_{-}, \Gamma_{a} \tau_{+}\right\rangle \mathbf{e}^{+}+2 \ell^{-2} A^{-1} u x^{a}\left\langle\Gamma_{+} \sigma_{-}, \Gamma_{a} \tau_{+}\right\rangle \mathbf{e}^{-} \\
& -2 \ell^{-1} x^{a}\left\langle\Gamma_{+} \sigma_{-}, \Gamma_{a} \tau_{+}\right\rangle \mathbf{e}^{z}+\left[-e^{-\frac{z}{\ell}}\left\langle\Gamma_{+} \sigma_{-}, \Gamma_{c} \tau_{+}\right\rangle+\ell^{-2} e^{\frac{z}{\ell}} x^{a} x^{b}\left\langle\Gamma_{+} \sigma_{-}, \Gamma_{a} \Gamma_{c} \Gamma_{b} \tau_{+}\right\rangle\right. \\
& \left.-2 \ell^{-2} A^{-2} u r e^{-\frac{z}{\ell}}\left\langle\Gamma_{+} \sigma_{-}, \Gamma_{c} \tau_{+}\right\rangle\right] \mathbf{e}^{c} \\
K\left(\epsilon_{2}, \epsilon_{4}\right)= & 2 \ell^{-1} e^{\frac{2 z}{\ell}} x^{a}\left\langle\sigma_{-}, \Gamma_{z a} \tau_{-}\right\rangle \mathbf{e}^{+}-2 \ell^{-1} A^{-1} u e^{\frac{z}{\ell}}\left\langle\sigma_{-}, \Gamma_{z a} \tau_{-}\right\rangle \mathbf{e}^{a} \\
K\left(\epsilon_{3}, \epsilon_{4}\right)= & -2 \ell^{-1} r A^{-1}\left\langle\tau_{+}, \Gamma_{+z} \tau_{-}\right\rangle \mathbf{e}^{+}+\left\langle\tau_{+}, \Gamma_{+z} \tau_{-}\right\rangle \mathbf{e}^{z}-\ell^{-1} x^{a} e^{\frac{z}{\ell}}\left\langle\tau_{+}, \Gamma_{a} \Gamma_{c} \Gamma_{+z} \tau_{-}\right\rangle \mathbf{e}^{c} \\
& +\left\langle\tau_{+}, \Gamma_{+i} \tau_{-}\right\rangle \mathbf{e}^{i}
\end{aligned}
$$

For $n>2$, we know that $\sigma_{-}=A \Gamma_{-z} \sigma_{+}^{\prime}$ and $\tau_{-}=A \Gamma_{-z} \tau_{+}^{\prime}$. Using these conditions, the bilinears can be written in the basis of Killing vectors of AdS given in appendix B as

$$
\begin{aligned}
K\left(\epsilon_{1}, \epsilon_{1}\right)= & 2\left\|\sigma_{+}\right\|^{2} \mathbf{e}^{-}=2\left\|\sigma_{+}\right\|^{2} \lambda^{-}, \\
K\left(\epsilon_{2}, \epsilon_{2}\right)= & -2\left(1+\ell^{-2} x^{2} e^{\frac{2 z}{\ell}}\right)\left\|\sigma_{-}\right\|^{2} \mathbf{e}^{+}+4 \ell^{-2} A^{-2} u^{2}\left\|\sigma_{-}\right\|^{2} \mathbf{e}^{-} \\
& -4 \ell^{-1} A^{-1} u\left\|\sigma_{-}\right\|^{2} \mathbf{e}^{z}+4 \ell^{-2} A^{-1} e^{\frac{z}{\ell}} u x_{a}\left\|\sigma_{-}\right\|^{2} \mathbf{e}^{a} \\
= & -2\left(4 \ell^{-1} M^{z+}+2 \lambda^{+}\right)\left\|\sigma_{+}^{\prime}\right\|^{2} \\
K\left(\epsilon_{3}, \epsilon_{3}\right)= & -4 \ell^{-2} A^{-2} r^{2} e^{-\frac{2 z}{\ell}}\left\|\tau_{+}\right\|^{2} \mathbf{e}^{+}+2\left(e^{-\frac{2 z}{\ell}}+\ell^{-2} x^{2}\right)\left\|\tau_{+}\right\|^{2} \mathbf{e}^{-} \\
& +4 \ell^{-1} A^{-1} r e^{-\frac{2 z}{\ell}}\left\|\tau_{+}\right\|^{2} \mathbf{e}^{z}-4 \ell^{-2} A^{-1} r e^{-\frac{z}{\ell}} x_{a}\left\|\tau_{+}\right\|^{2} \mathbf{e}^{a} \\
= & \left(4 \ell^{-1} M^{z-}+2 \lambda^{-}\right)\left\|\tau_{+}\right\|^{2} \\
K\left(\epsilon_{4}, \epsilon_{4}\right)= & -2 e^{\frac{2 z}{\ell}}\left\|\tau_{-}\right\|^{2} \mathbf{e}^{+}=-4\left\|\tau_{+}^{\prime}\right\|^{2} \lambda^{+} \\
K\left(\epsilon_{2}, \epsilon_{1}\right)= & 4 \ell^{-1} u\left\langle\sigma_{+}^{\prime}, \sigma_{+}\right\rangle \mathbf{e}^{-}-2 A\left\langle\sigma_{+}^{\prime}, \sigma_{+}\right\rangle \mathbf{e}^{z}+2 A \ell^{-1} e^{\frac{z}{\ell}} x^{a}\left\langle\sigma_{+}^{\prime}, \Gamma_{a} \Gamma_{c} \sigma_{+}\right\rangle \mathbf{e}^{c} \\
& -2 A\left\langle\sigma_{+}^{\prime}, \Gamma_{z i} \sigma_{+}\right\rangle \mathbf{e}^{i} \\
= & -2\left(\ell^{-1} M^{+-}+\lambda^{z}\right)\left\langle\sigma_{+}^{\prime}, \sigma_{+}\right\rangle-\ell^{-1} M^{a b}\left\langle\sigma_{+}^{\prime}, \Gamma_{a b} \sigma_{+}\right\rangle-2 A\left\langle\sigma_{+}^{\prime}, \Gamma_{z i} \sigma_{+}\right\rangle \mathbf{e}^{i} \\
K\left(\epsilon_{3}, \epsilon_{1}\right)= & -2 \ell^{-1} x^{a}\left\langle\Gamma_{a z} \tau_{+}, \sigma_{+}\right\rangle \mathbf{e}^{-}+2 \ell^{-1} A^{-1} r e^{-\frac{z}{\ell}}\left\langle\tau_{+}, \Gamma_{z a} \sigma_{+}\right\rangle \mathbf{e}^{a} \\
= & -2 \ell^{-1} M^{-a}\left\langle\Gamma_{a z} \tau_{+}, \sigma_{+}\right\rangle
\end{aligned}
$$




$$
\begin{aligned}
K\left(\epsilon_{4}, \epsilon_{1}\right)= & -2 A e^{\frac{z}{\ell}}\left\langle\tau_{+}^{\prime}, \Gamma_{z a} \sigma_{+}\right\rangle \mathbf{e}^{a}=-2 \lambda^{a}\left\langle\tau_{+}^{\prime}, \Gamma_{z a} \sigma_{+}\right\rangle \\
K\left(\epsilon_{2}, \epsilon_{3}\right)= & 4 \ell^{-2} r x^{a}\left\langle\sigma_{+}^{\prime}, \Gamma_{z a} \tau_{+}\right\rangle \mathbf{e}^{+}+4 \ell^{-2} u x^{a}\left\langle\sigma_{+}^{\prime}, \Gamma_{z a} \tau_{+}\right\rangle \mathbf{e}^{-} \\
& -4 A \ell^{-1} x^{a}\left\langle\sigma_{+}^{\prime}, \Gamma_{z a} \tau_{+}\right\rangle \mathbf{e}^{z}+\left[-2 A e^{-\frac{z}{\ell}}\left\langle\sigma_{+}^{\prime}, \Gamma_{z c} \tau_{+}\right\rangle\right. \\
& +2 A \ell^{-2} e^{\frac{z}{\ell}} x^{a} x^{b}\left\langle\sigma_{+}^{\prime}, \Gamma_{z a} \Gamma_{c} \Gamma_{b} \tau_{+}\right\rangle \\
& \left.-4 \ell^{-2} A^{-1} u r e^{-\frac{z}{\ell}}\left\langle\sigma_{+}^{\prime}, \Gamma_{z c} \tau_{+}\right\rangle\right] \mathbf{e}^{c} \\
= & -\left(4 \ell^{-1} M^{z a}+2 \lambda^{a}\right)\left\langle\sigma_{+}^{\prime}, \Gamma_{z a} \tau_{+}\right\rangle \\
K\left(\epsilon_{2}, \epsilon_{4}\right)= & -4 A^{2} \ell^{-1} e^{\frac{2 z}{\ell}} x^{a}\left\langle\sigma_{+}^{\prime}, \Gamma_{z a} \tau_{+}^{\prime}\right\rangle \mathbf{e}^{+}+4 A \ell^{-1} u e^{\frac{z}{\ell}}\left\langle\sigma_{+}^{\prime}, \Gamma_{z a} \tau_{+}^{\prime}\right\rangle \mathbf{e}^{a} \\
= & -4 \ell^{-1} M^{+a}\left\langle\sigma_{+}^{\prime}, \Gamma_{z a} \tau_{+}^{\prime}\right\rangle \\
K\left(\epsilon_{3}, \epsilon_{4}\right)= & 4 \ell^{-1} r\left\langle\tau_{+}, \tau_{+}^{\prime}\right\rangle \mathbf{e}^{+}-2 A\left\langle\tau_{+}, \tau_{+}^{\prime}\right\rangle \mathbf{e}^{z}+2 A \ell^{-1} x^{a} e^{\frac{z}{\ell}}\left\langle\tau_{+}, \Gamma_{a} \Gamma_{c} \tau_{+}^{\prime}\right\rangle \mathbf{e}^{c} \\
& +2 A\left\langle\tau_{+}, \Gamma_{z i} \tau_{+}^{\prime}\right\rangle \mathbf{e}^{i} \\
= & 2\left(-\lambda^{z}+\ell^{-1} M^{+-}\right)\left\langle\tau_{+}, \tau_{+}^{\prime}\right\rangle-\ell^{-1} M^{a b}\left\langle\tau_{+}, \Gamma_{a b} \tau_{+}^{\prime}\right\rangle+2 A\left\langle\tau_{+}, \Gamma_{z i} \tau_{+}^{\prime}\right\rangle \mathbf{e}^{i}
\end{aligned}
$$

From the above expressions it is straightforward to read the anti-commutator of any two odd generators of the superalgebra. Of course, for $n=3$ one should neglect all the terms which involve isometries that carry a $\mathbf{e}^{a}$ frame index.

\section{C.2 Spinorial Lie derivative}

The computation of the commutator of even and odd generators requires the evaluation of the spinorial Lie derivative of the Killing spinors along the isometries of AdS. In particular, the non-vanishing spinorial Lie derivatives of $\epsilon_{1}\left(\sigma_{+}\right)$along the isometries of AdS are as follows

$$
\begin{aligned}
\mathcal{L}_{\lambda^{z}} \epsilon_{1} & \left(\sigma_{+}\right)=\frac{1}{2} \ell^{-1} \epsilon_{1}\left(\sigma_{+}\right), & \mathcal{L}_{M^{+-}} \epsilon_{1}\left(\sigma_{+}\right) & =\frac{1}{2} \epsilon_{1}\left(\sigma_{+}\right), \\
\mathcal{L}_{M^{+a}} \epsilon_{1}\left(\sigma_{+}\right) & =-\frac{1}{2} \epsilon_{4}\left(A \Gamma_{-a} \sigma_{+}\right), & \mathcal{L}_{M^{a b}} \epsilon_{1}\left(\sigma_{+}\right) & =-\frac{1}{2} \epsilon_{1}\left(\Gamma_{a b} \sigma_{+}\right), \\
\mathcal{L}_{M^{z+}} \epsilon_{1}\left(\sigma_{+}\right) & =\frac{1}{2} \epsilon_{2}\left(A \Gamma_{-z} \sigma_{+}\right), & \mathcal{L}_{M^{z a}} \epsilon_{1}\left(\sigma_{+}\right) & =-\frac{1}{2} \epsilon_{3}\left(\Gamma_{z a} \sigma_{+}\right) .
\end{aligned}
$$

The non-vanishing spinorial Lie derivatives of $\epsilon_{2}\left(\sigma_{-}\right)$are

$$
\begin{aligned}
\mathcal{L}_{\lambda^{-}} \epsilon_{2}\left(\sigma_{-}\right) & =-\ell^{-1} \epsilon_{1}\left(A^{-1} \Gamma_{+z} \sigma_{-}\right), & \mathcal{L}_{\lambda^{a}} \epsilon_{2}\left(\sigma_{-}\right) & =-\ell^{-1} \epsilon_{4}\left(\Gamma_{a z} \sigma_{-}\right), \\
\mathcal{L}_{\lambda^{z}} \epsilon_{2}\left(\sigma_{-}\right) & =-\frac{1}{2} \ell^{-1} \epsilon_{2}\left(\sigma_{-}\right), & \mathcal{L}_{M^{+-}} \epsilon_{2}\left(\sigma_{-}\right) & =-\frac{1}{2} \epsilon_{2}\left(\sigma_{-}\right), \\
\mathcal{L}_{M^{a b}} \epsilon_{2}\left(\sigma_{-}\right) & =-\frac{1}{2} \epsilon_{2}\left(\Gamma_{a b} \sigma_{-}\right), & \mathcal{L}_{M^{-a}} \epsilon_{2}\left(\sigma_{-}\right) & =-\frac{1}{2} \epsilon_{3}\left(A^{-1} \Gamma_{+a} \sigma_{-}\right), \\
\mathcal{L}_{M^{z-}} \epsilon_{2}\left(\sigma_{-}\right) & =\frac{1}{2} \epsilon_{1}\left(A^{-1} \Gamma_{+z} \sigma_{-}\right), & \mathcal{L}_{M^{z a}} \epsilon_{2}\left(\sigma_{-}\right) & =\frac{1}{2} \epsilon_{4}\left(\Gamma_{a z} \sigma_{-}\right) .
\end{aligned}
$$

Similarly the non-vanishing spinorial Lie derivatives of $\epsilon_{3}\left(\tau_{+}\right)$are

$$
\begin{aligned}
\mathcal{L}_{\lambda^{+}} \epsilon_{3}\left(\tau_{+}\right) & =-\ell^{-1} \epsilon_{4}\left(A \Gamma_{-z} \tau_{+}\right), & \mathcal{L}_{\lambda^{a}} \epsilon_{3}\left(\tau_{+}\right) & =-\ell^{-1} \epsilon_{1}\left(\Gamma_{a z} \tau_{+}\right), \\
\mathcal{L}_{\lambda^{z}} \epsilon_{3}\left(\tau_{+}\right) & =-\frac{1}{2} \ell^{-1} \epsilon_{3}\left(\tau_{+}\right), & \mathcal{L}_{M^{+-}} \epsilon_{3}\left(\tau_{+}\right) & =\frac{1}{2} \epsilon_{3}\left(\tau_{+}\right), \\
\mathcal{L}_{M^{+a}} \epsilon_{3}\left(\tau_{+}\right) & =-\frac{1}{2} \epsilon_{2}\left(A \Gamma_{-a} \tau_{+}\right), & \mathcal{L}_{M^{a b}} \epsilon_{3}\left(\tau_{+}\right) & =-\frac{1}{2} \epsilon_{3}\left(\Gamma_{a b} \tau_{+}\right), \\
\mathcal{L}_{M^{z+}} \epsilon_{3}\left(\tau_{+}\right) & =\frac{1}{2} \epsilon_{4}\left(A \Gamma_{-z} \tau_{+}\right), & \mathcal{L}_{M^{z a}} \epsilon_{3}\left(\tau_{+}\right) & =\frac{1}{2} \epsilon_{1}\left(\Gamma_{a z} \tau_{+}\right),
\end{aligned}
$$


and those of $\epsilon_{4}\left(\tau_{-}\right)$are

$$
\begin{aligned}
\mathcal{L}_{\lambda^{z}} \epsilon_{4}\left(\tau_{-}\right) & =\frac{1}{2} \ell^{-1} \epsilon_{4}\left(\tau_{-}\right), & \mathcal{L}_{M^{+-}} \epsilon_{4}\left(\tau_{-}\right) & =-\frac{1}{2} \epsilon_{4}\left(\tau_{-}\right), \\
\mathcal{L}_{M^{-a}} \epsilon_{4}\left(\tau_{-}\right) & =-\frac{1}{2} \epsilon_{1}\left(A^{-1} \Gamma_{+a} \tau_{-}\right), & \mathcal{L}_{M^{a b}} \epsilon_{4}\left(\tau_{-}\right) & =-\frac{1}{2} \epsilon_{4}\left(\Gamma_{a b} \tau_{-}\right), \\
\mathcal{L}_{M^{z-}} \epsilon_{4}\left(\tau_{-}\right) & =\frac{1}{2} \epsilon_{3}\left(A^{-1} \Gamma_{+z} \tau_{-}\right), & \mathcal{L}_{M^{z a}} \epsilon_{4}\left(\tau_{-}\right) & =-\frac{1}{2} \epsilon_{2}\left(\Gamma_{z a} \tau_{-}\right) .
\end{aligned}
$$

\section{$\mathrm{D} \quad \mathrm{AdS}_{3} \mathrm{KSAs}$ for $N<14$}

D.1 $N=N_{\sigma}=10$

The 4 -form $\alpha$ is dual to a 1 -form. Because of this, the $\mathrm{SO}(5)$ automorphisms of the KSA can be used to choose the 1-form to lie in the 5-th direction, i.e. the only non-vanishing component of $\alpha$ is $\alpha_{1234}$. Next consider the commutator

$$
\left[\tilde{V}_{12}, \tilde{V}_{35}\right]
$$

Using

$$
\tilde{V}_{r s}=\frac{1}{2} \epsilon^{A B}\left\{Q_{A r}, Q_{B s}\right\}
$$

and after expressing first $\tilde{V}_{12}$ and then $\tilde{V}_{35}$ in terms of $Q$ 's, an application of the superJacobi identities reveals that

$$
\alpha_{1234} \tilde{V}_{45}=0
$$

Taking the commutator with $Q_{A 4}$ and using the fact that all $Q$ 's are linearly independent, we find that $\alpha_{1234}=0$ and so $\alpha=0$. As a result the $\mathrm{KSA}$ is $\mathfrak{o s p}(5,2)$. This is in agreement with the general analysis presented in section 3 for the $\mathrm{AdS}_{3}$ backgrounds.

D.2 $N=N_{\sigma}=12$

The 4 -form $\alpha$ is dual to a 2 -form. Using $\mathrm{SO}(6)$ automorphisms of the $\mathrm{KSA},{ }^{*} \alpha$ can be brought into the canonical form

$$
{ }^{*} \alpha=\lambda e^{1} \wedge e^{2}+\lambda_{2} e^{3} \wedge e^{4}+\lambda_{3} e^{5} \wedge e^{6}
$$

for some constants $\lambda_{1}, \lambda_{2}$ and $\lambda_{3}$.

It remains to determine these constants. For this consider the commutator

$$
\left[\tilde{V}_{12}, \tilde{V}_{35}\right]
$$

After using (D.2) to express first $\tilde{V}_{12}$ and then $\tilde{V}_{35}$ in terms of the $Q$ 's and applying the super-Jacobi identity in both cases, we find that

$$
-\lambda_{3} \tilde{V}_{54}+\lambda_{2} \tilde{V}_{36}=0
$$


The commutators of the above expression with $Q_{A 5}$ and $Q_{A 3}$ reveal that

$$
\lambda_{3}-\lambda_{2} \lambda_{1}=0, \quad \lambda_{2}-\lambda_{3} \lambda_{1}=0 .
$$

A similar calculation involving the commutator $\left[\tilde{V}_{34}, \tilde{V}_{15}\right]$ leads to

$$
\lambda_{1}-\lambda_{3} \lambda_{2}=0 \text {. }
$$

The solutions of these conditions are either that $\lambda_{1}=\lambda_{2}=\lambda_{3}=0$ or $\lambda_{1}^{2}=\lambda_{2}^{2}=\lambda_{3}^{2}=1$ and $\lambda_{1}=\lambda_{3} \lambda_{2}$. In the former case the superalgebra is $\mathfrak{o s p}(6 \mid 2)$ and in the latter case a real form of $\mathfrak{s l}(3 \mid 2)$ with even part $\mathfrak{s p}(2) \oplus \mathfrak{u}(3)$. The choices of signs of $\lambda$ 's denote the different embeddings of $\mathfrak{u}(3)$ in $\mathfrak{s o}(6)$. Again there is the possibility that the KSA is the non-simple superalgebra $\mathfrak{c s l}(3 \mid 2 ; 7)$. These results are in agreement with those in section 3.

Open Access. This article is distributed under the terms of the Creative Commons Attribution License (CC-BY 4.0), which permits any use, distribution and reproduction in any medium, provided the original author(s) and source are credited.

\section{References}

[1] V.G. Kac, A Sketch of Lie Superalgebra Theory, Commun. Math. Phys. 53 (1977) 31 [INSPIRE].

[2] W. Nahm, Supersymmetries and their Representations, Nucl. Phys. B 135 (1978) 149 [INSPIRE].

[3] A. Strominger, AdS $S_{2}$ quantum gravity and string theory, JHEP 01 (1999) 007 [hep-th/9809027] [INSPIRE].

[4] U. Gran, J.B. Gutowski and G. Papadopoulos, On supersymmetric Anti-de-Sitter, de-Sitter and Minkowski flux backgrounds, Class. Quant. Grav. 35 (2018) 065016 [arXiv:1607.00191] [INSPIRE].

[5] R. D'Auria and P. Fré, Spontaneous Generation of Osp(4/8) Symmetry in the Spontaneous Compactification of D =11 Supergravity, Phys. Lett. B 121 (1983) 141 [INSPIRE].

[6] B.S. Acharya, J.M. Figueroa-O'Farrill, C.M. Hull and B.J. Spence, Branes at conical singularities and holography, Adv. Theor. Math. Phys. 2 (1999) 1249 [hep-th/9808014] [INSPIRE].

[7] J.P. Gauntlett, R.C. Myers and P.K. Townsend, Supersymmetry of rotating branes, Phys. Rev. D 59 (1998) 025001 [hep-th/9809065] [INSPIRE].

[8] M. Blau, J.M. Figueroa-O'Farrill, C. Hull and G. Papadopoulos, A New maximally supersymmetric background of IIB superstring theory, JHEP 01 (2002) 047 [hep-th/0110242] [INSPIRE].

[9] J.M. Figueroa-O'Farrill, On the supersymmetries of Anti-de Sitter vacua, Class. Quant. Grav. 16 (1999) 2043 [hep-th/9902066] [INSPIRE].

[10] D. Montgomery and H. Samelson, Transformation groups of spheres, Ann. Math. 44 (1943) 454.

[11] J. Simons, On the transitivity of holonomy systems, Ann. Math. 76 (1962) 213. 
[12] J.B. Gutowski and G. Papadopoulos, Supersymmetry of AdS and flat backgrounds in M-theory, JHEP 02 (2015) 145 [arXiv: 1407.5652] [INSPIRE].

[13] S.W. Beck, J.B. Gutowski and G. Papadopoulos, Supersymmetry of AdS and flat IIB backgrounds, JHEP 02 (2015) 020 [arXiv: 1410.3431] [INSPIRE].

[14] S. Beck, J.B. Gutowski and G. Papadopoulos, Supersymmetry of IIA warped flux AdS and flat backgrounds, JHEP 09 (2015) 135 [arXiv:1501.07620] [INSPIRE].

[15] J.M. Figueroa-O'Farrill, P. Meessen and S. Philip, Supersymmetry and homogeneity of M-theory backgrounds, Class. Quant. Grav. 22 (2005) 207 [hep-th/0409170] [InSPIRE].

[16] J.M. Figueroa-O'Farrill, E. Hackett-Jones and G. Moutsopoulos, The Killing superalgebra of ten-dimensional supergravity backgrounds, Class. Quant. Grav. 24 (2007) 3291 [hep-th/0703192] [INSPIRE].

[17] S.W. Beck, J.B. Gutowski and G. Papadopoulos, Geometry and supersymmetry of heterotic warped flux AdS backgrounds, JHEP 07 (2015) 152 [arXiv:1505.01693] [INSPIRE].

[18] J.M. Figueroa-O'Farrill and G. Papadopoulos, Maximally supersymmetric solutions of ten-dimensional and eleven-dimensional supergravities, JHEP 03 (2003) 048 [hep-th/0211089] [INSPIRE].

[19] J. Figueroa-O'Farrill and N. Hustler, The homogeneity theorem for supergravity backgrounds, JHEP 10 (2012) 014 [arXiv:1208.0553] [INSPIRE].

[20] A.S. Haupt, S. Lautz and G. Papadopoulos, AdS 4 backgrounds with $N>16$ supersymmetries in 10 and 11 dimensions, JHEP 01 (2018) 087 [arXiv:1711.08280] [INSPIRE].

[21] S.W. Beck, J.B. Gutowski and G. Papadopoulos, AdS $S_{5}$ backgrounds with 24 supersymmetries, JHEP 06 (2016) 126 [arXiv: 1601.06645] [INSPIRE].

[22] H. Lin, O. Lunin and J.M. Maldacena, Bubbling AdS space and 1/2 BPS geometries, JHEP 10 (2004) 025 [hep-th/0409174] [INSPIRE].

[23] E. D'Hoker, M. Gutperle, A. Karch and C.F. Uhlemann, Warped $A d S_{6} \times S^{2}$ in Type IIB supergravity I: Local solutions, JHEP 08 (2016) 046 [arXiv:1606.01254] [INSPIRE].

[24] J. Gutowski and G. Papadopoulos, On supersymmetric AdS solutions in 10 and 11 dimensions, JHEP 12 (2017) 009 [arXiv: 1702.06048] [INSPIRE].

[25] U. Gran, G. Papadopoulos and C. von Schultz, Supersymmetric geometries of IIA supergravity I, JHEP 05 (2014) 024 [arXiv:1401.6900] [INSPIRE].

[26] U. Gran, GAMMA: A Mathematica package for performing gamma matrix algebra and Fierz transformations in arbitrary dimensions, hep-th/0105086 [INSPIRE]. 